\title{
Rigorous lubrication approximation
}

\author{
LORENZO GIACOMELLI ${ }^{\dagger}$ \\ Dipartimento Me.Mo.Mat., Università di Roma "La Sapienza", \\ Via Scarpa 16, 00161 Roma, Italy \\ AND \\ FELIX OTTO \\ Institut für Angewandte Mathematik, Universität Bonn, \\ Wegelerstr. 10, 53115 Bonn, Germany \\ [Received 11 January 2003 and in revised form 7 May 2003]
}

\begin{abstract}
We rigorously carry out a lubrication approximation for a liquid thin film which spreads on a solid, driven by surface tension. We consider a two-dimensional Darcy liquid as simple model case. Of particular interest to us is the codimension-two free boundary, i.e. the triple junctions where solid, liquid and vapor meet. In the considered regime of complete wetting, the contact angle vanishes throughout the evolution. We show in particular that this contact-angle condition is preserved in the lubrication approximation.
\end{abstract}

\section{Contents}

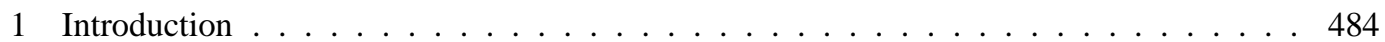

1.1 The Darcy flow in half space . . . . . . . . . . . . . . . . . . . . . . . . . . . . . . . . . . . . . . . . . . . . . . . .

1.2 Mass and energy $\ldots \ldots \ldots \ldots$. . . . . . . . . . . . . . . . . . . . . . . . . . . . . . . . . . . . . . . . . . . .

1.3 The thin-film regime $\ldots \ldots \ldots \ldots$. . . . . . . . . . . . . . . . . . . . . . . . . . . . . . . . . . . . . . . . . . . . . . .

1.4 The thin-film equation $\ldots \ldots \ldots \ldots$. . . . . . . . . . . . . . . . . . . . . . . . . . . . . . . . . . . . .

2 The main result . . . . . . . . . . . . . . . . . . . . . . . . . 490

2.1 Rigorous statement of result . . . . . . . . . . . . . . . . . . . 490

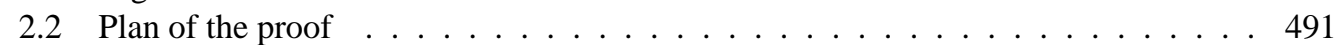

3 Basic uniform bounds . . . . . . . . . . . . . . . . . . . . . . . . . . . . . . . 494

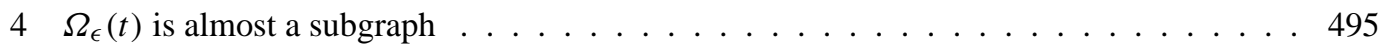

5 Convergence of $h_{\epsilon} \quad \ldots \ldots \ldots \ldots$. . . . . . . . . . . . . . . . . . . . . . 499

6 Convergence of $v_{\epsilon}$ and $\hat{\pi}_{\epsilon} \ldots \ldots \ldots \ldots \ldots$

7 Horizontal and vertical variations . . . . . . . . . . . . . . . . . . . . . 508

8 Pressure neutrality and passage to the limit . . . . . . . . . . . . . . . . . . . . 517

9 Recovering the equation . . . . . . . . . . . . . . . . . . . . . 523

10 Post-processing I: Regularity and zero contact-angle . . . . . . . . . . . . . . . . . . 524

11 Post-processing II: The "Neumann-type" formulation . . . . . . . . . . . . . . . . . . . 527

†Email: giacomelli@dmmm.uniroma1.it

Email: otto@riemann.iam.uni-bonn.de 


\section{Introduction}

Liquid films are characterized by a separation of length scales: the (evolving) region occupied by the liquid is thin and gently sloping. The lubrication approximation capitalizes on this separation of scales to substantially reduce the complexity of the appropriate bulk fluid model, thus allowing for a more efficient study of the free surface (see [14] for a review on the subject). We are in particular interested in a viscous liquid film which (slowly) spreads on a solid under the driving force of surface tension. This is the case in the so-called regime of "complete wetting", where the solid-air surface tension equals the sum of solid-liquid and liquid-air surface tensions.

In this paper, we give a rigorous justification of the lubrication approximation in the simplest possible case: a surface-tension driven Darcy flow of a thin film. Also, we only treat the twodimensional case. This set-up is relevant for the Hele-Shaw cell. Of particular interest to us is the "codimension-two boundary", i.e. the boundary of the set wetted by the liquid film. In our twodimensional setting, it is formed by the triple junction where liquid, solid and vapor meet. The static or equilibrium contact angle is determined by Young's law (cf. [10]). In the complete wetting regime, the equilibrium contact angle is zero. For a Darcy flow, the equilibrium contact angle is preserved throughout the evolution. To our knowledge, this is the first rigorous justification of a lubrication approximation in the presence of a codimension-two boundary. The delicate part is the passage to the limit in the zero contact-angle condition at the triple junction. We hope that our technique may apply to more general situations such as the surface-tension driven Stokes flow with, say, the Navier slip condition.

\subsection{The Darcy flow in half space}

In this section, we introduce the two-dimensional Darcy flow which is our starting point. This model, which is motivated by the flow in a Hele-Shaw cell, is already non-dimensionalized. It describes the evolution of a region $\Omega(t)$ in the upper half plane $\{(x, y): y>0\}$, and we think of $\Omega(t)$ as touching the fixed boundary $\{(x, y): y=0\}$. To specify the evolution means to specify how the normal velocity $V(t)$ of the free boundary $\partial \Omega(t) \cap\{y>0\}$ depends on its shape. Let $\tau, v$, and $\kappa$ denote respectively the unit tangent, outer unit normal and the curvature of $\partial \Omega(t)$, with the understanding that the pair $(\tau, v)$ has positive orientation, $\kappa v=-\partial_{\tau} \tau$ (cf. Fig. 1). $V(t)$ is taken with respect to the outer normal $v$. The first set of assumptions says that the free boundary $\partial \Omega(t) \cap\{y>0\}$ of

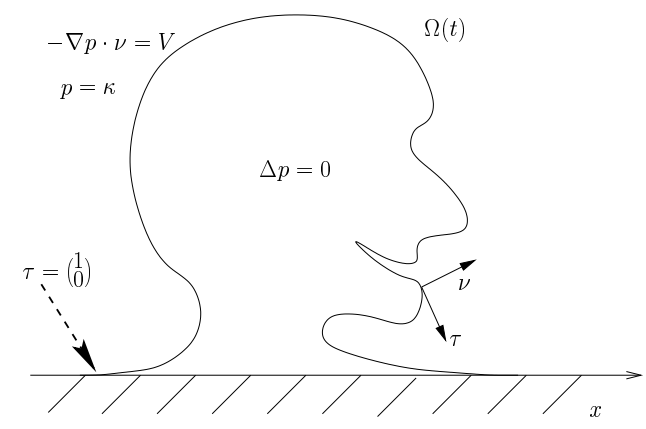

FIG. 1. The Darcy flow in half space in complete wetting regime. 
the domain $\Omega(t)$ filled by the liquid is advected by a divergence-free velocity field $u(t)$ with slip boundary condition at the wetted set $\partial \Omega(t) \cap\{y=0\}$ :

$$
\left\{\begin{aligned}
\nabla \cdot u(t) & =0 & & \text { in } \Omega(t), \\
\left({ }^{0}\right) \cdot u(t) & =0 & & \text { on } \partial \Omega(t) \cap\{y=0\}, \\
\nu \cdot u(t) & =V & & \text { on } \partial \Omega(t) \cap\{y>0\} .
\end{aligned}\right.
$$

These are the "kinetic conditions". The second set of assumptions, the "dynamic conditions", is more interesting: The velocity is the negative gradient of a pressure $p(t)$ in the domain filled by the liquid (this is Darcy's law), the pressure balances surface tension, as given by the curvature, at the free boundary $\partial \Omega(t) \cap\{y>0\}$, and the free boundary is tangential to the fixed boundary at the triple junctions $\partial(\partial \Omega(t) \cap\{y>0\})$ (this is Young's law or the zero contact-angle condition):

$$
\left\{\begin{aligned}
u(t) & =-\nabla p(t) & & \text { in } \Omega(t), \\
\kappa & =p(t) & & \text { on } \partial \Omega(t) \cap\{y>0\}, \\
\left(\begin{array}{l}
1 \\
0
\end{array}\right) \cdot \tau & =1 & & \text { at } \partial(\partial \Omega(t) \cap\{y>0\}) .
\end{aligned}\right.
$$

\subsection{Mass and energy}

There are two fundamental quantities associated with a generic domain $\Omega$ : its area and the length of $\partial \Omega \cap\{y>0\}$ minus the length of $\partial \Omega \cap\{y=0\}$,

$$
\begin{aligned}
M(\Omega) & =\mathcal{L}^{2}(\Omega), \\
E(\Omega) & =\mathcal{H}^{1}(\partial \Omega \cap\{y>0\})-\mathcal{H}^{1}(\partial \Omega \cap\{y=0\}) .
\end{aligned}
$$

The first quantity is proportional to the mass of the liquid filling $\Omega$. In the complete wetting regime under consideration, the second quantity is (up to an additive constant) the total interfacial energy of the configuration $\Omega$. We now investigate the behavior of these quantities under the above dynamics. The kinetic conditions (1.1) by themselves imply that $M$ is conserved,

$$
\frac{\mathrm{d}}{\mathrm{d} t} M(\Omega(t))=\int_{\partial \Omega(t) \cap\{y>0\}} V(t)=\int_{\Omega(t)} \nabla \cdot u(t)=0,
$$

and that $E$ evolves according to

$$
\begin{aligned}
\frac{\mathrm{d}}{\mathrm{d} t} E(\Omega(t)) & =\int_{\partial \Omega(t) \cap\{y>0\}} \tau \cdot \mathrm{D} u(t) \tau-\int_{\partial \Omega(t) \cap\{y=0\}}\left(\begin{array}{l}
1 \\
0
\end{array}\right) \cdot \mathrm{D} u(t)\left(\begin{array}{l}
1 \\
0
\end{array}\right) \\
& =\int_{\partial \Omega(t) \cap\{y>0\}} \kappa V(t)-\int_{\partial(\partial \Omega(t) \cap\{y>0\})} \sqrt{\frac{1-\left(\begin{array}{l}
1 \\
0
\end{array}\right) \cdot \tau}{1+\left(\begin{array}{l}
1 \\
0
\end{array}\right) \cdot \tau}} V(t) .
\end{aligned}
$$

From the calculation (1.4) we see that the first variation of the energy $E$ of a generic domain $\Omega$ (of which we do not assume the contact-angle condition in (1.2) is given by

$$
\left\langle d E_{\Omega}, \tilde{V}\right\rangle=\int_{\partial \Omega \cap\{y>0\}} \kappa \tilde{V}-\int_{\partial(\partial \Omega \cap\{y>0\})} \sqrt{\frac{1-\left(\begin{array}{l}
1 \\
0
\end{array}\right) \cdot \tau}{1+\left(\begin{array}{l}
1 \\
0
\end{array}\right) \cdot \tau}} \tilde{V}
$$

for all kinetically admissible $\tilde{V}$, where we call $\tilde{V}: \partial \Omega \cap\{y>0\} \rightarrow \mathbb{R}$ kinetically admissible if $\int_{\partial \Omega \cap\{y>0\}} \tilde{V}=0$. This shows that the contact-angle condition is indeed an equilibrium condition. 
Therefore, it is a "natural" (Neumann) boundary condition rather than an "essential" (Dirichlet) boundary condition. This will be important later on. The dynamic conditions 1.2 (and the kinetic conditions (1.1) again) ensure that $E$ does not increase:

$$
\begin{gathered}
\frac{\mathrm{d}}{\mathrm{d} t} E(\Omega(t)) \stackrel{1.4,[1.2}{=} \int_{\partial \Omega(t) \cap\{y>0\}} p V(t) \\
\stackrel{1.1}{=} \int_{\Omega(t)} \nabla p(t) \cdot u(t) \\
\\
=\int_{\Omega(t)}^{1.2}|u(t)|^{2} .
\end{gathered}
$$

In fact, the evolution can be written as a gradient flow of $E$ (see [11]).

From this analysis we infer the following interpretation of the evolution $t \mapsto \Omega(t)$ : The last dynamic condition states that $\Omega(t)$ relaxes instantaneously at the triple junctions $\partial(\partial \Omega(t) \cap\{y>0\})$ to balance both contributions to $E$ (i.e. the three surface energies). On the other hand, the first two dynamic conditions ensure that, away from $\partial(\partial \Omega(t) \cap\{y>0\}), \Omega(t)$ evolves to reduce the first part of $E$ (i.e. the liquid-air surface energy). Hence the evolution can be seen as the limiting case of the combination of a slow and a fast relaxation.

\subsection{The thin-film regime}

Let $X$ and $Y$ denote the typical horizontal, respectively vertical length scale of $\Omega(t)$. The lubrication approximation is based on the separation of scales

$$
\epsilon=\frac{Y}{X} \ll 1
$$

We now heuristically infer the typical time scale $T$ on which $t \mapsto \Omega(t)$ changes. Since $\Omega$ is advected by the velocity $u$ (cf. the last equation in (1.1)), we have for its horizontal and vertical components

$$
u \cdot\left(\begin{array}{l}
1 \\
0
\end{array}\right) \sim \frac{X}{T}, \quad u \cdot\left(\begin{array}{l}
0 \\
1
\end{array}\right) \sim \frac{Y}{T} .
$$

In view of Darcy's law (cf. the first condition in 1.2 ), this implies

$$
\frac{\partial p}{\partial x} \sim \frac{X}{T}, \quad \frac{\partial p}{\partial y} \sim \frac{Y}{T} .
$$

Because of (1.7), the vertical pressure gradient is thus much smaller than the horizontal one, so that $p$ is essentially a function of $x$ alone. Hence we may infer from the first part of (1.8) that

$$
p-(\text { mean of } p) \sim \frac{X^{2}}{T} .
$$

In view of the balance of pressure and surface tension (cf. the second condition in (1.2)), this yields for the curvature

$$
\kappa-(\text { mean of } \kappa) \sim \frac{X^{2}}{T} \text {. }
$$


On the other hand, (1.7) implies that $\kappa$ scales as

$$
\kappa \sim \frac{Y}{X^{2}} .
$$

But in view of Young's law (cf. the last condition in (1.2)), the mean of the curvature vanishes so that we obtain from equating (1.10) and (1.11):

$$
T=\frac{X^{4}}{Y} .
$$

We now encode 1.77 with the help of quantities which can be controlled in terms of their initial value. According to (1.3), the mass $M$ is one of such quantities; as we shall see in Section 3 , another such quantity is the second moment $Q$ of the domain $\Omega$ :

$$
Q(\Omega)=\int_{\Omega} \frac{1}{2}\left(x^{2}+y^{2}\right) .
$$

In terms of scaling, we expect

$$
M(\Omega) \sim X Y, \quad Q(\Omega) \stackrel{[1.7]}{\sim} X^{3} Y .
$$

Hence we define $X$ and $Y$ through

$$
\frac{1}{X Y} M\left(\Omega_{0}\right)=1, \quad \frac{1}{X^{3} Y} Q\left(\Omega_{0}\right)=1,
$$

that is,

$$
X:=\left(\frac{Q\left(\Omega_{0}\right)}{M\left(\Omega_{0}\right)}\right)^{1 / 2}, \quad Y:=\left(\frac{M\left(\Omega_{0}\right)^{3}}{Q\left(\Omega_{0}\right)}\right)^{1 / 2}, \quad T \stackrel{1.12)}{=}\left(\frac{Q\left(\Omega_{0}\right)^{5}}{M\left(\Omega_{0}\right)^{7}}\right)^{1 / 2},
$$

and the somewhat vague condition 1.7 is replaced by

$$
\epsilon:=\frac{M\left(\Omega_{0}\right)^{2}}{Q\left(\Omega_{0}\right)} \ll 1
$$

In the lubrication approximation, $\Omega$ is described in terms of its rescaled height function $\hat{h}$ :

$$
\Omega(T \hat{t})=\{(X \hat{x}, Y \hat{y}): 0<\hat{y}<\hat{h}(\hat{t}, \hat{x})\} .
$$

Not surprisingly, 1.15 is insufficient to ensure that $\Omega(T \hat{t})$ is even close to a subgraph. We need an additional condition. Notice that if 1.16 holded, we would have

$$
\begin{aligned}
\frac{X}{Y^{2}} E(\Omega(T \hat{t})) & =\frac{1}{\epsilon^{2}} \int_{-\infty}^{\infty}\left(\sqrt{1+\left(\epsilon \frac{\partial \hat{h}}{\partial \hat{x}}(\hat{t}, \hat{x})\right)^{2}}-1\right) \mathrm{d} \hat{x} \\
& \approx \int_{-\infty}^{\infty} \frac{1}{2}\left(\frac{\partial \hat{h}}{\partial \hat{x}}(\hat{t}, \hat{x})\right)^{2} \mathrm{~d} \hat{x} \quad \text { provided } \quad \frac{\partial \hat{h}}{\partial \hat{x}}=O(1)
\end{aligned}
$$

This suggests that the condition

$$
\frac{X}{Y^{2}} E(\Omega(T \hat{t}))=O(1) \quad \text { in } \epsilon
$$


is enough to ensure that $\Omega(T \hat{t})$ is at least close to a subgraph:

$$
\Omega(T \hat{t}) \approx\{(X \hat{x}, Y \hat{y}): 0<\hat{y}<\hat{h}(\hat{t}, \hat{x})\} .
$$

We shall make this rigorous in Section 4. According to (1.6), $E(\Omega(t))$ is non-increasing; hence it is sufficient to require $(1.18)$ only initially. In view of $(1.14)$, this translates into the condition

$$
\frac{Q\left(\Omega_{0}\right)^{3} E\left(\Omega_{0}\right)^{2}}{M\left(\Omega_{0}\right)^{7}}=O(1) \quad \text { in } \epsilon .
$$

This is the additional condition defining the thin-film regime.

In view of $(1.17),(1.18)$ is a version of the standard assumption in lubrication theory that the film be gently sloped. Notice that 1.18 amounts only to an average bound on the slope $\partial \hat{h} / \partial \hat{x}$, which is at the origin of technical difficulties in our rigorous treatment. But this average bound is probably the only "honest" bound-in the sense that it is the only one preserved by the evolution, because it is encoded in an energy bound. In particular, the property of being a subgraph is not preserved.

\subsection{The thin-film equation}

We give a brief heuristic derivation of the thin-film equation, which also guides some of the rigorous treatment. Observe that $[1.19]$ in particular implies

$$
\hat{h}(\hat{t}, \hat{x}) \approx \int_{0}^{\infty} \chi_{\Omega(T \hat{t})}(X \hat{x}, Y \hat{y}) \mathrm{d} \hat{y},
$$

so that the kinetic conditions $(1.1)$ turn to leading order into

$$
\frac{1}{T} \frac{\partial \hat{h}}{\partial \hat{t}}+\frac{1}{X} \frac{\partial}{\partial \hat{x}}\left(\hat{h} \bar{u} \cdot\left(\begin{array}{l}
1 \\
0
\end{array}\right)\right)=0
$$

where $\bar{u}$ is the vertical average of the velocity. Recall that the anisotropy of the pressure gradient (cf. (1.8) in the regime 1.7 implies that to leading order, $p$ is a function of $\hat{x}$ alone. Therefore, Darcy's law (cf. (1.2)) can be vertically averaged:

$$
\bar{u} \cdot\left(\begin{array}{l}
1 \\
0
\end{array}\right)=-\frac{1}{X} \frac{\partial p}{\partial \hat{x}} .
$$

On the other hand, we infer from 1.19) in the regime 1.7 that to leading order

$$
\kappa=-\frac{Y}{X^{2}} \frac{\partial^{2} \hat{h}}{\partial \hat{x}^{2}} .
$$

Hence the balance of pressure and surface tension (cf. 1.2 ) yields

$$
p=-\frac{Y}{X^{2}} \frac{\partial^{2} \hat{h}}{\partial \hat{x}^{2}} .
$$

Combining (1.21), 1.22) with (1.23) and using (1.12), we obtain

$$
\frac{\partial \hat{h}}{\partial \hat{t}}+\frac{\partial}{\partial \hat{x}}\left(\hat{h} \frac{\partial^{3} \hat{h}}{\partial \hat{x}^{3}}\right)=0 \quad \text { in }\{\hat{h}>0\} .
$$


We observe that this limit process is a singular limit. Indeed, consider the codimension-one freeboundary problem for $\partial \Omega(t) \cap\{y>0\}$ in $(1.1)-(1.2)$. Linearization around a flat interface yields a third-order parabolic (and therefore non-local) operator. On the other hand, the thin-film equation 1.24 is a local fourth-order parabolic evolution for the height function $\hat{h}(\hat{t}, \hat{x})$. The limit process is well-understood on the level of careful asymptotic expansions (cf. [1, 14]).

The above heuristic argument, and the more careful asymptotic expansions, do not address the fate of the codimension-two boundary $\partial(\partial \Omega \cap\{y>0\})$ and the contact-angle condition at these triple junctions. The main merit of our rigorous result is that it also treats $\partial\{\hat{h}>0\}$. In short, our result can be formulated as follows:

Assume we are in the thin-film regime in the sense of (1.15) and $(1.20)$. Then

$$
\Omega(T \hat{t}) \approx\{(X \hat{x}, Y \hat{y}): 0<\hat{y}<\hat{h}(\hat{t}, \hat{x})\},
$$

where $\hat{h}(\hat{t}, \hat{x}) \geqslant 0$ is a solution of

$$
\left\{\begin{array}{cl}
\frac{\partial \hat{h}}{\partial \hat{t}}+\frac{\partial}{\partial \hat{x}}\left(\hat{h} \frac{\partial^{3} \hat{h}}{\partial \hat{x}^{3}}\right)=0 \quad \text { in }\{\hat{h}>0\}, \\
\hat{h}=\frac{\partial \hat{h}}{\partial \hat{x}}=\hat{h} \frac{\partial^{3} \hat{h}}{\partial \hat{x}^{3}}=0 & \text { on } \partial\{\hat{h}>0\} .
\end{array}\right.
$$

Notice that 1.25 can indeed be read as a free-boundary problem for $\partial\{\hat{h}>0\}$ : On the "free domain" $U:=\{h>0\}$ in space-time, $\hat{h}$ satisfies a fourth-order parabolic equation. Three boundary conditions are imposed on $\partial U \cap\{t>0\}$ : the trivial "defining condition" $\hat{h}=0$, the zero contactangle condition $\partial \hat{h} / \partial \hat{x}=0$, and the zero mass-flux condition $\hat{h} \partial^{3} \hat{h} / \partial \hat{x}^{3}=0$. This would be one condition too much on a fixed domain, but $\partial U$ evolves with a spatial speed given by $\partial^{3} \hat{h} / \partial \hat{x}^{3}$, as can be read off from the first equation in 1.25 .

The main difficulty in the proof is the passage to the limit in the zero contact-angle condition. The other two conditions at the free boundary are more robust: the defining condition $\hat{h}=0$ is well-controlled by a priori estimates, whereas the zero flux is well-encoded in an appropriate weak formulation. The main idea is to treat the contact-angle condition as a natural (Neumann) boundary condition rather than an essential (Dirichlet) boundary condition. From the point of view of physics, this is obvious since the contact-angle condition is a static equilibrium condition, as shown in (1.5). The fact that this static equilibrium condition also holds throughout the evolution is natural from the gradient flow perspective as explained by the authors in [11]: The time discretization of the gradient flow, which comes in the form of a sequence of variational problems, clearly shows that the contact angle is an outcome of (instantaneous) energy relaxation at the triple point rather than a constraint on the space of shapes $\hat{h}$. On this time-discrete level, the passage to the limit in the angle condition has been carried out in [11].

The second technical difficulty comes from the lack of control of the "topology" of $\Omega(t)$ or $\{\hat{h}(\hat{t}, \cdot)>0\}$. It is indeed conjectured that the number of connected components may increase through the formation of a "pinching" singularity [2]. Our weak notion of solution for $[1.25]$ is valid throughout a singularity formation. But the lack of topology control only permits a weak control of the relative pressure $p$, as already alluded to in (1.9). Indeed, we only control the gradient of the pressure on the evolving and possibly pinching domain $\Omega(t)$. 


\section{The main result}

\section{$2.1 \quad$ Rigorous statement of result}

In this subsection, we give the precise formulation of our result. We first clarify the notion of solutions. In short, we require all regularity on $\Omega$, but little regularity on $\hat{h}(\hat{t}, \hat{x})$. Throughout the paper,

$$
\begin{aligned}
& \Omega(t) \text { is a smooth global solution of } 11.1-1.2 \text { such that the boundary of } \\
& \qquad \Omega^{0}(t):=\Omega(t) \cup\{y \leqslant 0\} \\
& \text { is a connected and continuously differentiable curve for all } t \geqslant 0 .
\end{aligned}
$$

In addition to regularity and compatibility with the complete wetting regime, (2.1) in particular rules out that a connected component of $\Omega(t)$ has empty intersection with $\{y=0\}$ : In other words, 2.1 forbids the detachment of liquid droplets from the film.

REMARK 2.1 In the absence of a codimension-two boundary (i.e. assuming that the initial wetted region $\partial \Omega_{0} \cap\{y=0\}$ coincides with $\{y=0\}$ ), Escher and Simonett [9] established local existence and uniqueness of classical solutions in any space dimension (cf. also Prokert [16]). The twodimensional case for subgraphs had been first considered by Duchon and Robert [8]. Global existence of smooth solution for the evolution in all of $\mathbb{R}^{2}$ (i.e. assuming that $\partial \Omega_{0} \cap\{y=0\}$ is empty) has been proved by Constantin and Pugh [7] provided $\partial \Omega_{0}$ is nearly circular. Of course, we do expect to have at least short-time existence of classical solutions for (1.1)-(1.2) (provided initial data are such that no waiting-time phenomenon occurs). But to the best of our knowledge such a result is not available, and would actually be an interesting subject of research.

We now introduce the notion of weak solution for 1.25 .

DEFINITION 1 A function $\hat{h}(\hat{t}, \hat{x}) \geqslant 0$ is called a solution of 1.25 if:

(i) $\hat{h} \in C\left([0, \infty) \times \mathbb{R}_{\hat{x}}\right) \cap L^{\infty}\left((0, \infty) ; H^{1}\left(\mathbb{R}_{\hat{x}}\right)\right)$;

(ii) $\partial^{3} \hat{h} / \partial \hat{x}^{3} \in L_{\mathrm{loc}}^{2}(\{\hat{h}>0\})$ and $\hat{h}^{1 / 2} \partial^{3} \hat{h} / \partial \hat{x}^{3} \in L^{2}(\{\hat{h}>0\})$;

(iii) $\partial^{2} \hat{h} / \partial \hat{x}^{2} \in L^{3}\left((0, \infty) \times \mathbb{R}_{\hat{x}}\right)$;

(iv) for all $\zeta \in C_{c}^{\infty}\left((0, \infty) \times \mathbb{R}_{\hat{x}}\right)$,

$$
\int_{0}^{\infty} \int_{-\infty}^{\infty} \hat{h} \frac{\partial \zeta}{\partial \hat{t}} \mathrm{~d} \hat{x} \mathrm{~d} \hat{t}=-\int_{0}^{\infty} \int_{-\infty}^{\infty}\left\{\frac{3}{2}\left(\frac{\partial \hat{h}}{\partial \hat{x}}\right)^{2} \frac{\partial^{2} \zeta}{\partial \hat{x}^{2}}+\hat{h} \frac{\partial \hat{h}}{\partial \hat{x}} \frac{\partial^{3} \zeta}{\partial \hat{x}^{3}}\right\} \mathrm{d} \hat{x} \mathrm{~d} \hat{t}
$$

REMARK 2.2 Definition 1 is a weak formulation for problem 1.25. Indeed, two integrations by parts of the right hand side of (2.2) (using (iii) to control the boundary terms) yield

$$
\int_{0}^{\infty} \int_{-\infty}^{\infty} \hat{h} \frac{\partial \zeta}{\partial \hat{t}} \mathrm{~d} \hat{x} \mathrm{~d} \hat{t}+\iint_{\{\hat{h}>0\}} \hat{h} \frac{\partial^{3} \hat{h}}{\partial \hat{x}^{3}} \frac{\partial \zeta}{\partial \hat{x}} \mathrm{~d} \hat{x} \mathrm{~d} \hat{t}=0
$$

for all $\zeta \in C_{c}^{\infty}\left((0, \infty) \times \mathbb{R}_{\hat{x}}\right)$. Furthermore, (ii) and (iii) imply that

$$
\lim _{\hat{x} \rightarrow \partial\{\hat{h}(\hat{t}, \cdot)>0\}}\left(h \frac{\partial^{3} \hat{h}}{\partial \hat{x}^{3}}\right)(\hat{t}, \hat{x})=0 \quad \text { and } \quad \hat{h}(\hat{t}, \cdot) \in C^{1, \beta}\left(\mathbb{R}_{\hat{x}}\right), \quad \beta=\frac{2}{3}
$$

for almost every $\hat{t}$, so that the free boundary conditions at $\partial\{\hat{h}>0\}$ are encoded. 
The thin-film equation in its many variants has been extensively studied in the recent years. Existence of solutions for (1.25) in one space dimension was proved in [6, 3] in bounded domains, and in [5] in unbounded domains (we refer to [13] for the higher-dimensional case and for an upto-date list of references). A by-product of our approach (cf. Theorem 2 ) is to provide an alternative proof of existence of solutions for (1.25), which unlike previous results does not make use of socalled "entropy estimates". As a consequence, the regularity which can be ascertained from (iii) is slightly weaker than that of the solutions obtained in [6, 3] (for those, (2.4) holds true for any $\beta<1$; $\beta=1$ is sharp). This potentially could make a difference since no uniqueness result is yet available.

We are now ready to formulate our main result:

THEOREM 1 For all $\delta>0$, there exists an $\epsilon(\delta)>0$ with the following property: If $\Omega(t)$ is a solution of the Darcy flow in the sense of 2.1] in the thin-film regime in the sense of

$$
\frac{Q\left(\Omega_{0}\right)^{3} E\left(\Omega_{0}\right)^{2}}{M\left(\Omega_{0}\right)^{7}} \leqslant \frac{1}{\delta} \text { and } \quad \frac{M\left(\Omega_{0}\right)^{2}}{Q\left(\Omega_{0}\right)} \leqslant \epsilon(\delta),
$$

then there exists a solution $\hat{h}(\hat{t}, \hat{x})$ of the thin-film equation in the sense of Definition 1 which is close to $\Omega(t)$ in the sense of

$$
\Omega(T \hat{t})\left\{\begin{array}{l}
\subset\{(X \hat{x}, Y \hat{y}): 0<\hat{y}<\hat{h}(\hat{t}, \hat{x})+\delta\} \\
\supset\{(X \hat{x}, Y \hat{y}): 0<\hat{y}<\hat{h}(\hat{t}, \hat{x})-\delta\}
\end{array}\right\} \quad \text { for all } 0 \leqslant \hat{t} \leqslant \frac{1}{\delta}
$$

Here, $X, Y$ and $T$ are defined in 1.14 . Furthermore, $\hat{h}$ is non-trivial in the sense that

$$
\int_{-\infty}^{\infty} \hat{h}(\hat{t}, \hat{x}) \mathrm{d} \hat{x}=1 \quad \text { for all } \hat{t} \geqslant 0
$$

\subsection{Plan of the proof}

We rescale 1.1 - 1.2 measuring both horizontal and vertical lengths in units of $X$, and time in units of $T$, with $X$ and $T$ defined by (1.14):

$$
\begin{aligned}
u_{\epsilon}(\hat{t}, \hat{x}, \check{y}) & :=\frac{T}{X} u(T \hat{t}, X \hat{x}, X \check{y}), \\
p_{\epsilon}(\hat{t}, \hat{x}, \check{y}) & :=\frac{T}{X^{2}} p(T \hat{t}, X \hat{x}, X \check{y}), \\
\Omega_{\epsilon}(\hat{t}) & :=\left\{(\hat{x}, \check{y}) \in \mathbb{R}_{\hat{x}} \times \mathbb{R}_{\check{y}}:(X \hat{x}, X \check{y}) \in \Omega(T \hat{t})\right\} .
\end{aligned}
$$

Then, with $\epsilon$ defined by $(1.15)$, we have for the rescaled initial configurations $\Omega_{0 \epsilon}$,

$$
\begin{aligned}
M\left(\Omega_{0 \epsilon}\right) & =\epsilon, \\
Q\left(\Omega_{0 \epsilon}\right) & =\epsilon, \\
E\left(\Omega_{0 \epsilon}\right) & =\frac{1}{X} E\left(\Omega_{0}\right),
\end{aligned}
$$

and $\Omega_{\epsilon}(\hat{t})$ evolves according to

$$
\left\{\begin{aligned}
\nabla \cdot u_{\epsilon}(\hat{t})=0 & \text { in } \Omega_{\epsilon}(\hat{t}), \\
\left(\begin{array}{c}
0 \\
-1
\end{array}\right) \cdot u_{\epsilon}(\hat{t})=0 & \text { on } \partial \Omega_{\epsilon}(\hat{t}) \cap\{\check{y}=0\}, \\
v \cdot u_{\epsilon}(\hat{t})=V & \text { on } \partial \Omega_{\epsilon}(\hat{t}) \cap\{\check{y}>0\},
\end{aligned}\right.
$$




$$
\left\{\begin{array}{rlrl}
u_{\epsilon}(\hat{t}) & =-\nabla p_{\epsilon}(\hat{t}) & & \text { in } \Omega_{\epsilon}(\hat{t}), \\
\frac{1}{\epsilon} \kappa & =p_{\epsilon}(\hat{t}) & & \text { on } \partial \Omega_{\epsilon}(\hat{t}) \cap\{\check{y}>0\}, \\
\left(\begin{array}{l}
1 \\
0
\end{array}\right) \cdot \tau=1 & & \text { at } \partial\left(\partial \Omega_{\epsilon}(\hat{t}) \cap\{\check{y}>0\}\right) .
\end{array}\right.
$$

The vertical length scale $Y$ is introduced by measuring vertical slices of $\Omega_{\epsilon}(\hat{t})$ in its units:

$$
\begin{array}{r}
h_{\epsilon}(\hat{t}, \hat{x}) \quad:=\frac{X}{Y} \int_{0}^{\infty} \chi_{\Omega_{\epsilon}(\hat{t})}(\hat{x}, \check{y}) \mathrm{d} \check{y} \\
\frac{1.14],[1.15}{\epsilon} \int_{0}^{\infty} \chi_{\Omega_{\epsilon}(\hat{t})}(\hat{x}, \check{y}) \mathrm{d} \check{y} .
\end{array}
$$

Since the area is conserved (cf. 1.3 ), we have

$$
\int_{\mathbb{R}_{\hat{x}}} h_{\epsilon}(\hat{t}, \hat{x}) \mathrm{d} \hat{x}=1 .
$$

We argue by contradiction, assuming that for a certain $\delta>0$ and any $\epsilon>0$ there exists a solution $\Omega(t)$ of the Darcy flow such that 2.5 holds and 2.6) is false for any solution of the thin-film equation. The second assumption in $(2.5)$ yields the existence of a sequence $\left\{\left(\Omega_{\epsilon}, p_{\epsilon}\right)\right\}_{\epsilon \downarrow 0}$ of solutions of (2.11)-2.12) such that 2.8 -2.10 hold true. In addition, the first assumption in (2.5) guarantees that

$$
\frac{1}{\epsilon^{2}} E\left(\Omega_{0 \epsilon}\right) \quad \text { is bounded for } \epsilon \downarrow 0 .
$$

Theorem 1 is now a simple consequence of the following two results:

THEOREM 2 Let $\left\{\left(\Omega_{\epsilon}, p_{\epsilon}\right)\right\}_{\epsilon \downarrow 0}$ be a sequence of solutions of problem $\sqrt{2.11}-\sqrt{2.12}$ in the sense of (2.1) such that (2.8), 2.9) and 2.14) hold, and let $h_{\epsilon}$ be defined by (2.13). There exists a subsequence such that for all $\tau<\infty$,

$$
h_{\epsilon} \stackrel{\epsilon \downarrow 0}{\longrightarrow} \hat{h} \quad \text { uniformly in }[0, \tau] \times \mathbb{R}_{\hat{x}}
$$

and $\hat{h}$ is a solution of 1.25 in the sense of Definition 1 In addition

$$
\int_{\mathbb{R}_{\hat{x}}} \hat{h}(\hat{t}, \hat{x}) \mathrm{d} \hat{x}=1 \quad \text { for all } \hat{t} \geqslant 0 .
$$

Proposition 1 Under the assumptions of Theorem 2, there exists a universal constant $C_{0}$ such that for $\epsilon$ sufficiently small

$$
\left\{\check{y} \in(0, \infty):(\hat{x}, \check{y}) \in \Omega_{\epsilon}(\hat{t})\right\}\left\{\begin{array}{l}
\subset\left(0, \epsilon h_{\epsilon}(\hat{t}, \hat{x})+C_{0} E\left(\Omega_{0 \epsilon}\right)\right) \\
\supset\left(0, \epsilon h_{\epsilon}(\hat{t}, \hat{x})-C_{0} E\left(\Omega_{0 \epsilon}\right)\right)
\end{array}\right.
$$

for any $(\hat{t}, \hat{x}) \in(0, \infty) \times \mathbb{R}_{\hat{x}}$.

The paper is devoted to the proof of Theorem 2 and Proposition 1 We now give an outline of the proof and its structure. 
- In Section 3 we recall the standard bounds on the mass $M$, the energy $E$, its dissipation $D$, and the second moment $Q$. These will be the uniform a priori bounds we rely upon throughout what follows.

- In Section 4 we show, as a consequence of the uniform bound on energy, that $\Omega_{\epsilon}(\hat{t})$ is close to a subgraph for small $\epsilon$, and that the portion of the line over which $\Omega_{\epsilon}(\hat{t})$ is not a subgraph is small in an appropriate sense. The results of this section in particular yield Proposition 1

- In Section $[5]$ we show that, for a subsequence, $h_{\epsilon}$ converges uniformly to a continuous function $\hat{h}$.

- In Section 6 we prove that the horizontal velocity $v_{\epsilon}=u_{\epsilon} \cdot\left(\begin{array}{l}1 \\ 0\end{array}\right)$ and the (suitably renormalized) relative pressure $p_{\epsilon}-\operatorname{mean}\left(p_{\epsilon}\right)$ converge weakly to a $\hat{v}$, respectively a $\hat{\pi}$. We argue that both limits only depend on $x$ and pass to the limit in the kinetic conditions and Darcy's law:

$$
\frac{\partial \hat{h}}{\partial \hat{t}}+\frac{\partial}{\partial \hat{x}}(\hat{h} \hat{v})=0 \quad \text { and } \quad \hat{v}=-\frac{\partial \hat{\pi}}{\partial \hat{x}} \quad \text { distributionally in }(0, \infty) \times \mathbb{R}_{\hat{x}}
$$

We are forced to consider the relative pressure $p_{\epsilon}-\operatorname{mean}\left(p_{\epsilon}\right)$ since we do not know how to control its mean mean $\left(p_{\epsilon}\right)$. Since we control the relative pressure through its gradient, we have to stay safely inside a connected component of $\{\hat{h}>0\}$, so that the Poincaré estimate does not deteriorate.

- Section 7 is the core of our proof. We encode the dynamic conditions by testing the original problem with two different types of infinitesimal variations, as described by a velocity $\tilde{u}$. The first type are vertical variations of the form $\tilde{u}=(0, \tilde{w}(\hat{x}))$. Using the convexity of the energy functional over the portion where $h_{\epsilon}$ is a graph, we encode this variation as an inequality. This makes it more robust for the passage to the limit. This first type of variation is already sufficient (cf. Section 9$\}$ to identify $\hat{v}=(\partial / \partial \hat{x})^{3} \hat{h}$ in the interior of $\{\hat{h}>0\}$. On the other hand, Young's law cannot be captured by variations which are necessarily confined inside the positivity set: We need a second type of variations, which are essentially horizontal $\tilde{u}=(v, 0)$ and mimic shifts of connected components of $\{\hat{h}>0\}$. Unfortunately, the construction of both test fields $\tilde{u}$ is technically involved since the portions over which $\Omega_{\epsilon}$ is not a subgraph have to be avoided.

- In Section 8 we pass to the limit in the two aforementioned variational formulations. To do so, we have to combine both formulations in such a way that the combination is oblivious to the absolute value of the pressure (we call this "pressure neutrality").

- In Section 9 we recover the thin-film equation, i.e. $\hat{v}=(\partial / \partial \hat{x})^{3} \hat{h}$ on $\{\hat{h}>0\}$, from the variational formulation of Section 8

- In Section 10 we recover the zero contact-angle condition, i.e. $\partial \hat{h} / \partial \hat{x}=0$ at $\partial\{\hat{h}>0\}$, from the variational formulation of Section 8

- In Section 11 we recover the "Neumann-type" formulation 2.2, which encompasses both the equation and the zero contact-angle condition.

We believe that this method can also be applied to the surface-tension driven Stokes flow with slip in the complete wetting regime. The higher-dimensional version of our result, save the many regularity issues (some of them yet open and fairly difficult), should in principle also be achievable by similar arguments. On the other hand, we doubt that our technique can be extended to the case of non-zero contact angle. Indeed, our "horizontal" variations (cf. Section 7), are not able to encode a nonzero contact angle. This reflects the higher degree of non-convexity of the non-zero contact-angle problem (cf. [15]). 
Notations. From now on we will be working with solutions of the rescaled flow (2.11)-(2.12), and therefore for notational convenience we remove hats and checks, letting $(t, x, y)=(\hat{t}, \hat{x}, \grave{y}), h=\hat{h}$. The superscript ' will denote differentiation with respect to the variable $x$.

\section{Basic uniform bounds}

We start by collecting the basic estimates for solutions $\left(\Omega_{\epsilon}, p_{\epsilon}\right)$ of the rescaled Darcy flow in terms of area, momentum, energy and dissipation rate. According to (2.8), 2.9] and (2.14), we introduce

$$
\begin{aligned}
M_{\epsilon}(t) & :=\frac{1}{\epsilon} \mathcal{L}^{2}\left(\Omega_{\epsilon}(t)\right), \\
Q_{\epsilon}(t) & :=\frac{1}{\epsilon} \int_{\Omega_{\epsilon}(t)} \frac{1}{2}\left(x^{2}+y^{2}\right) \mathrm{d} x \mathrm{~d} y, \\
E_{\epsilon}(t) & :=\frac{1}{\epsilon^{2}}\left[\mathcal{H}^{1}\left(\partial \Omega_{\epsilon}(t) \cap\{y>0\}\right)-\mathcal{H}^{1}\left(\partial \Omega_{\epsilon}(t) \cap\{y=0\}\right)\right], \\
D_{\epsilon}(t) & :=\frac{1}{\epsilon} \int_{\Omega_{\epsilon}(t)}\left|u_{\epsilon}(t)\right|^{2} .
\end{aligned}
$$

LEMMA 3.1

$$
\begin{aligned}
M_{\epsilon}(t)=1 & \text { for all } t \in(0, \infty), \\
\sup _{t \in(0, \infty)}\left\{\mathrm{e}^{-t} Q_{\epsilon}(t)\right\} & \text { is bounded for } \epsilon \downarrow 0, \\
\sup _{t \in(0, \infty)} E_{\epsilon}(t) \leqslant E_{\epsilon}(0) & \text { is bounded for } \epsilon \downarrow 0, \\
\int_{0}^{\infty} D_{\epsilon}(t) \mathrm{d} t \leqslant E_{\epsilon}(0) & \text { is bounded for } \epsilon \downarrow 0 .
\end{aligned}
$$

Proof. We rewrite for better readability and further reference the dynamic boundary conditions

$$
\begin{array}{rlrl}
\frac{1}{\epsilon} \kappa & =p_{\epsilon}(t) \quad & \text { on } \partial \Omega_{\epsilon}(t) \cap\{y>0\}, \\
\tau & =\left(\begin{array}{l}
1 \\
0
\end{array}\right) \quad \text { at } \partial\left(\partial \Omega_{\epsilon}(t) \cap\{y>0\}\right),
\end{array}
$$

Darcy's law

$$
u_{\epsilon}(t)=-\nabla p_{\epsilon}(t) \quad \text { in } \Omega_{\epsilon}(t)
$$

and the kinetic conditions

$$
\begin{aligned}
\nabla \cdot u_{\epsilon}(t)=0 & \text { in } \Omega_{\epsilon}(t), \\
u_{\epsilon}(t) \cdot\left(\begin{array}{c}
0 \\
-1
\end{array}\right)=0 & \text { on } \partial \Omega_{\epsilon}(t) \cap\{y=0\}, \\
u_{\epsilon}(t) \cdot v=V & \text { on } \partial \Omega_{\epsilon}(t) \cap\{y>0\} .
\end{aligned}
$$

Lemma 3.1 is an immediate consequence of the initial bounds and the following estimates for time derivatives:

$$
\frac{\mathrm{d} M_{\epsilon}}{\mathrm{d} t}(t)=0, \quad \frac{\mathrm{d} Q_{\epsilon}}{\mathrm{d} t}(t) \leqslant Q_{\epsilon}(t)+\frac{1}{2} D_{\epsilon}(t), \quad \frac{\mathrm{d} E_{\epsilon}}{\mathrm{d} t}(t)=-D_{\epsilon}(t)
$$


The first one and the last one are the rescaled versions of (1.3), respectively (1.6). For the middle one we use the Cauchy-Schwarz and Young inequalities:

$$
\begin{aligned}
\frac{\mathrm{d} Q_{\epsilon}}{\mathrm{d} t}(t) & =\frac{1}{\epsilon} \int_{\partial \Omega_{\epsilon}(t) \cap\{y>0\}} V \frac{1}{2}\left(x^{2}+y^{2}\right) \\
& \stackrel{3.10]}{=} \frac{1}{\epsilon} \int_{\partial \Omega_{\epsilon}(t) \cap\{y>0\}} u_{\epsilon}(t) \cdot v \frac{1}{2}\left(x^{2}+y^{2}\right) \\
& \stackrel{3.9}{=} \frac{1}{\epsilon} \int_{\Omega_{\epsilon}(t)} \nabla \cdot\left[\frac{1}{2}\left(x^{2}+y^{2}\right) u_{\epsilon}(t)\right] \\
& \leqslant \frac{13.8}{\epsilon} \int_{\Omega_{\epsilon}(t)}\left(\begin{array}{l}
x \\
y
\end{array}\right) \cdot u_{\epsilon}(t) \\
& \leqslant \frac{1}{\epsilon} \int_{\Omega_{\epsilon}(t)} \frac{1}{2}\left(x^{2}+y^{2}\right) \mathrm{d} x \mathrm{~d} y+\frac{1}{\epsilon} \int_{\Omega_{\epsilon}(t)} \frac{1}{2}\left|u_{\epsilon}(t)\right|^{2} .
\end{aligned}
$$

\section{4. $\Omega_{\epsilon}(t)$ is almost a subgraph}

Since the property of being a subgraph is not necessarily preserved by the evolution, we did not want to assume it. The goal of this section is to show, for bounded $E_{\epsilon}(t)$ and $\epsilon \ll 1$, that nevertheless $\Omega_{\epsilon}(t)$ is close to the subgraph of $\epsilon h_{\epsilon}(t)$, and that it coincides with the subgraph of $\epsilon h_{\epsilon}$ over a set with a small complement. More precisely, we have the following:

Proposition 4.1 There exists a universal constant $C_{0}$ such that for $\epsilon$ sufficiently small

$$
\left\{y \in(0, \infty):(x, y) \in \Omega_{\epsilon}(t)\right\}\left\{\begin{array}{l}
\subset\left(0, \epsilon h_{\epsilon}(t, x)+C_{0} \epsilon^{2} E_{\epsilon}(t)\right) \\
\supset\left(0, \epsilon h_{\epsilon}(t, x)-C_{0} \epsilon^{2} E_{\epsilon}(t)\right)
\end{array}\right.
$$

for any $(t, x) \in(0, \infty) \times \mathbb{R}$. Furthermore, for any $0<\delta \leqslant 1$ and any $t \in(0, \infty)$ there exists an open set $U_{\delta, \epsilon}(t)$ with

$$
\begin{gathered}
\left\{y \in(0, \infty):(x, y) \in \Omega_{\epsilon}(t)\right\}=\left(0, \epsilon h_{\epsilon}(t, x)\right) \quad \text { for all } x \in U_{\delta, \epsilon}(t), \\
\int_{U_{\delta, \epsilon}(t)} \frac{1}{2}\left(h_{\epsilon}^{\prime}(t)\right)^{2} \leqslant \sqrt{1+\delta^{2}} E_{\epsilon}(t),
\end{gathered}
$$

such that $\mathbb{R}-U_{\delta, \epsilon}(t)$ is small in the sense of

$$
\begin{aligned}
\mathcal{L}^{1}\left(\mathbb{R}-U_{\delta, \epsilon}(t)\right) & \leqslant C_{0} \frac{\epsilon^{2}}{\delta^{2}} E_{\epsilon}(t), \\
\left|\mathrm{d} h_{\epsilon}(t)\right|\left(\mathbb{R}-U_{\delta, \epsilon}(t)\right) & \leqslant C_{0} \frac{\epsilon}{\delta} E_{\epsilon}(t) .
\end{aligned}
$$

Remark 4.2 Here $\left|\mathrm{d} h_{\epsilon}(t)\right|$ denotes the total variation measure of $h_{\epsilon}(t)$. Proposition 4.1 implies in particular Proposition 1 .

Proof. By the assumption 2.1 , the boundary of the set $\Omega_{\epsilon}^{0}(t)$ is connected and continuously differentiable. Let $(-\infty, \infty) \ni s \mapsto\left(x_{\epsilon}(t, s), y_{\epsilon}(t, s)\right)$ be a parametrization of this curve by arc 
length, oriented so that $\lim _{s \rightarrow \pm \infty} x_{\epsilon}(t, s)= \pm \infty$. Then the energy $E_{\epsilon}(t)$ can be expressed in terms of this curve:

$$
\epsilon^{2} E_{\epsilon}(t)=\int_{-\infty}^{\infty}\left(1-\frac{\mathrm{d} x_{\epsilon}}{\mathrm{d} s}(t, s)\right) \mathrm{d} s .
$$

We start by identifying the maximal subset $U_{\epsilon}(t)$ of the $x$-axis over which $\Omega_{\epsilon}(t)$ can be written as a subgraph. Consider

$$
\begin{aligned}
\mathbb{R}-U_{\epsilon}(t) & =\left\{x_{\epsilon}(t, s): \frac{\mathrm{d} x_{\epsilon}}{\mathrm{d} s}(t, s) \leqslant 0\right\}, \\
P_{\epsilon}(t) & =\left\{s \in(-\infty, \infty): x_{\epsilon}(t, s) \in U_{\epsilon}(t)\right\},
\end{aligned}
$$

and observe that both $P_{\epsilon}(t)$ and $U_{\epsilon}(t)$ are open sets. Also, for $x \in U_{\epsilon}(t)$ there exists a unique $s$ with $x_{\epsilon}(t, s)=x$. Therefore, by definition of the curve and $h_{\epsilon}(t)$,

$$
\begin{aligned}
\left\{y \in(0, \infty):\left(x_{\epsilon}(t, s), y\right) \in \Omega_{\epsilon}(t)\right\}=\left(0, y_{\epsilon}(t, s)\right) & \text { for all } s \in P_{\epsilon}(t), \\
y_{\epsilon}(t, s)=\epsilon h_{\epsilon}\left(t, x_{\epsilon}(t, s)\right) & \text { for all } s \in P_{\epsilon}(t) .
\end{aligned}
$$

In particular, $h_{\epsilon}(t)$ is continuously differentiable on $U_{\epsilon}(t)$ with

$$
\left.\begin{array}{rl}
\frac{\mathrm{d} x_{\epsilon}}{\mathrm{d} s}(t, s) & >0 \\
\frac{\mathrm{d} y_{\epsilon}}{\mathrm{d} s}(t, s) & =\epsilon h_{\epsilon}^{\prime}\left(t, x_{\epsilon}(t, s)\right) \frac{\mathrm{d} x_{\epsilon}}{\mathrm{d} s}(t, s) \\
\left(\frac{\mathrm{d} x_{\epsilon}}{\mathrm{d} s}\right)^{2}+\left(\frac{\mathrm{d} y_{\epsilon}}{\mathrm{d} s}\right)^{2} & =1
\end{array}\right\} \quad \text { for all } s \in P_{\epsilon}(t),
$$

which implies that

$$
\frac{\mathrm{d} x_{\epsilon}}{\mathrm{d} s} \sqrt{1+\left(\epsilon h_{\epsilon}^{\prime}(t)\right)^{2}}=1 \quad \text { for all } s \in P_{\epsilon}(t) .
$$

We also note for further reference the inequality

$$
\left(\frac{\mathrm{d} y_{\epsilon}}{\mathrm{d} s}\right)^{2}=1-\left(\frac{\mathrm{d} x_{\epsilon}}{\mathrm{d} s}\right)^{2} \leqslant 2\left(1-\frac{\mathrm{d} x_{\epsilon}}{\mathrm{d} s}\right) .
$$

Another way to characterize $U_{\epsilon}(t)$ uses the marginal $\lambda_{\epsilon}(t)$ of the Hausdorff measure along the curve with respect to the $x$-variable:

$$
\int_{-\infty}^{\infty} \zeta\left(x_{\epsilon}(t, s)\right) \mathrm{d} s=\int_{\mathbb{R}} \zeta \mathrm{d} \lambda_{\epsilon}(t) .
$$

Indeed, we will show that $\lambda_{\epsilon}(t)$ has density $\sqrt{1+\left(\epsilon h_{\epsilon}^{\prime}(t)\right)^{2}}$ with respect to the Lebesgue measure on $U_{\epsilon}(t)$ :

$$
\lambda_{\epsilon}(t, B)=\int_{B} \sqrt{1+\left(\epsilon h_{\epsilon}^{\prime}(t, x)\right)^{2}} \mathrm{~d} x \text { for all Borel sets } B \subset U_{\epsilon}(t),
$$

and that on the other hand, $\lambda_{\epsilon}(t)$ is large with respect to the Lebesgue measure on its complement:

$$
\lambda_{\epsilon}(t, B) \geqslant 3 \mathcal{L}^{1}(B) \quad \text { for all Borel sets } B \subset \mathbb{R}-U_{\epsilon}(t) .
$$


For (4.12), let $\zeta$ be a continuous function with support in $U_{\epsilon}(t)$. Then, by definition of $U_{\epsilon}(t)$, $\zeta\left(x_{\epsilon}(t, s)\right)=0$ for $x \in(-\infty, \infty)-P_{\epsilon}(t)$, so that

$$
\begin{aligned}
\int_{\mathbb{R}} \zeta d \lambda_{\epsilon}(t) & =\int_{P_{\epsilon}(t)} \zeta\left(x_{\epsilon}(t, s)\right) \mathrm{d} s \\
& \stackrel{4.9}{=} \int_{P_{\epsilon}(t)} \zeta\left(x_{\epsilon}(t, s)\right) \sqrt{1+\left(\epsilon h_{\epsilon}^{\prime}\left(t, x_{\epsilon}(t, s)\right)\right)^{2}} \frac{\mathrm{d} x_{\epsilon}}{\mathrm{d} s}(t, s) \underline{\mathrm{s}} .
\end{aligned}
$$

Since $x_{\epsilon}$ maps $P_{\epsilon}(t)$ strictly monotonically onto $U_{\epsilon}(t)$, we have

$$
\int_{P_{\epsilon}(t)} \zeta\left(x_{\epsilon}(t, s)\right) \sqrt{1+\left(\epsilon h_{\epsilon}^{\prime}\left(t, x_{\epsilon}(t, s)\right)\right)^{2}} \frac{\mathrm{d} x_{\epsilon}}{\mathrm{d} s}(t, s) \mathrm{d} s=\int_{U_{\epsilon}(t)} \zeta(x) \sqrt{1+\left(\epsilon h_{\epsilon}^{\prime}(t, x)\right)^{2}} \mathrm{~d} x .
$$

This string of identities implies 4.12). have

For 4.13 , it is enough to show that for any $x_{0} \in \mathbb{R}-U_{\epsilon}(t)$ and all sufficiently small $\delta>0$ we

$$
\lambda_{\epsilon}\left(t,\left(x_{0}-\delta, x_{0}+\delta\right)\right) \geqslant 3 \mathcal{L}^{1}\left(\left(x_{0}-\delta, x_{0}+\delta\right)\right) .
$$

By definition of $U_{\epsilon}(t)$, there exists an $s_{0} \in(-\infty, \infty)$ with $x_{\epsilon}\left(t, s_{0}\right)=x_{0}$ and $\frac{\mathrm{d} x_{\epsilon}}{\mathrm{d} s}\left(t, s_{0}\right) \leqslant 0$. We distinguish two cases. First, assume that $\frac{\mathrm{d} x_{\epsilon}}{\mathrm{d} s}\left(t, s_{0}\right)<0$. Since $\lim _{s \rightarrow \pm \infty} x_{\epsilon}(t, s)= \pm \infty$, there exist $s_{-}<s_{0}<s_{+}$with $x_{\epsilon}\left(t, s_{-}\right)=x_{\epsilon}\left(t, s_{+}\right)=x_{0}$ and $\frac{\mathrm{d} x_{\epsilon}}{\mathrm{d} s}\left(t, s_{-}\right), \frac{\mathrm{d} x_{\epsilon}}{\mathrm{d} s}\left(t, s_{+}\right)>0$. Provided $\delta \ll 1$, there exist open and disjoint intervals $P_{-} \ni s_{-}, P_{0} \ni s_{0}$ and $P_{+} \ni s_{+}$such that $x_{\epsilon}$ maps each of them strictly monotonically onto $\left(x_{0}-\delta, x_{0}+\delta\right)$. Therefore

$$
\begin{aligned}
\lambda_{\epsilon}\left(t,\left(x_{0}-\delta, x_{0}+\delta\right)\right) & \geqslant \int_{P_{-}} 1 \mathrm{~d} s+\int_{P_{0}} 1 \mathrm{~d} s+\int_{P_{+}} 1 \mathrm{~d} s \\
& \geqslant \int_{P_{-}}\left|\frac{\mathrm{d} x_{\epsilon}}{\mathrm{d} s}\right| \mathrm{d} s+\int_{P_{0}}\left|\frac{\mathrm{d} x_{\epsilon}}{\mathrm{d} s}\right| \mathrm{d} s+\int_{P_{+}}\left|\frac{\mathrm{d} x_{\epsilon}}{\mathrm{d} s}\right| \mathrm{d} s \\
& =3 \mathcal{L}^{1}\left(\left(x_{0}-\delta, x_{0}+\delta\right)\right) .
\end{aligned}
$$

Case 2 is when $\frac{\mathrm{d} x_{\epsilon}}{\mathrm{d} s}\left(t, s_{0}\right)=0$. In this case, for $\delta \ll 1$ we have

$$
x_{\epsilon}\left(t,\left(s_{0}-3 \delta, s_{0}+3 \delta\right)\right) \subset\left(x_{0}-\delta, x_{0}+\delta\right),
$$

so that

$$
\lambda_{\epsilon}\left(t,\left(x_{0}-\delta, x_{0}+\delta\right)\right) \geqslant \int_{\left(s_{0}-3 \delta, s_{0}+3 \delta\right)} 1 \mathrm{~d} s=6 \delta=3 \mathcal{L}^{1}\left(\left(x_{0}-\delta, x_{0}+\delta\right)\right) .
$$

This completes the proof of 4.13 ).

According to (4.12) and (4.13), $\lambda_{\epsilon}(t)$ dominates the Lebesgue measure:

$$
\lambda_{\epsilon}(t, B) \geqslant \mathcal{L}^{1}(B) \quad \text { for all Borel sets } B \text {. }
$$

On the other hand,

$$
\begin{gathered}
\lambda_{\epsilon}(t, B) \stackrel{\text { 4.11 }}{=} \int_{x_{\epsilon}(t, s) \in B} \mathrm{~d} s=\mathcal{L}^{1}(B)+\int_{x_{\epsilon}(t, s) \in B}\left(1-\frac{\mathrm{d} x_{\epsilon}}{\mathrm{d} s}(t, s)\right) \mathrm{d} s \\
\stackrel{4.6}{\leqslant} \mathcal{L}^{1}(B)+\epsilon^{2} E_{\epsilon}(t) \quad \text { for all Borel sets } B .
\end{gathered}
$$


Together with 4.13, this implies

$$
\mathcal{L}^{1}\left(\mathbb{R}-U_{\epsilon}(t)\right) \stackrel{4.13}{\leqslant} \frac{1}{3} \lambda_{\epsilon}\left(t, \mathbb{R}-U_{\epsilon}(t)\right) \stackrel{4.14}{\leqslant} \frac{1}{3}\left[\mathcal{L}^{1}\left(\mathbb{R}-U_{\epsilon}(t)\right)+\epsilon^{2} E_{\epsilon}(t)\right],
$$

so that

$$
\mathcal{L}^{1}\left(\mathbb{R}-U_{\epsilon}(t)\right) \leqslant \frac{\epsilon^{2}}{2} E_{\epsilon}(t) .
$$

We first prove 4.1. Obviously, it is enough to show that

$$
\left|y_{\epsilon}\left(t, s_{1}\right)-y_{\epsilon}\left(t, s_{2}\right)\right| \leqslant C_{0} \epsilon^{2} E_{\epsilon}(t) \quad \text { for all } s_{1}<s_{2} \text { such that } x_{\epsilon}\left(t, s_{1}\right)=x_{\epsilon}\left(t, s_{2}\right) \text {. }
$$

According to (4.6, we have

$$
\begin{aligned}
\left|y_{\epsilon}\left(t, s_{1}\right)-y_{\epsilon}\left(t, s_{2}\right)\right| & \leqslant \int_{s_{1}}^{s_{2}}\left|\frac{\mathrm{d} y_{\epsilon}}{\mathrm{d} s}(t, s)\right| \mathrm{d} s \stackrel{4.10}{\leqslant} \sqrt{2} \int_{s_{1}}^{s_{2}} \sqrt{1-\frac{\mathrm{d} x_{\epsilon}}{\mathrm{d} s}(t, s)} \mathrm{d} s \\
& \leqslant\left(2\left(s_{2}-s_{1}\right) \epsilon^{2} E_{\epsilon}(t)\right)^{1 / 2} .
\end{aligned}
$$

Let now $b_{1}=\inf _{s \in\left(s_{1}, s_{2}\right)} x_{\epsilon}(t, s), b_{2}=\sup _{s \in\left(s_{1}, s_{2}\right)} x_{\epsilon}(t, s)$. Since $\left(b_{1}, b_{2}\right) \subset \mathbb{R}-U_{\epsilon}(t)$, we see that

$$
\begin{aligned}
s_{2}-s_{1} & =\int_{s_{1}}^{s_{2}} \chi_{\left(b_{1}, b_{2}\right)}\left(x_{\epsilon}(t, s)\right) \mathrm{d} s \stackrel{4.11}{\leqslant} \lambda_{\epsilon}\left(t,\left(b_{1}, b_{2}\right)\right) \\
& \stackrel{4.14}{\leqslant} \mathcal{L}^{1}\left(\mathbb{R}-U_{\epsilon}(t)\right)+\epsilon^{2} E_{\epsilon}(t) \stackrel{4.15}{\leqslant} \frac{3}{2} \epsilon^{2} E_{\epsilon}(t) .
\end{aligned}
$$

Inserting 4.18 into 4.17 we obtain 4.16.

For $0<\delta \leqslant 1$, we introduce the open set

$$
U_{\delta, \epsilon}(t)=\left\{x \in U_{\epsilon}(t):\left|\epsilon h_{\epsilon}^{\prime}(t, x)\right|<\delta\right\} .
$$

Since $U_{\delta, \epsilon}(t) \subset U_{\epsilon}(t), 4.7-4.8$ imply 4.2. According to 4.12,

$$
\lambda_{\epsilon}(t, B) \leqslant \sqrt{1+\delta^{2}} \mathcal{L}^{1}(B) \text { for all Borel sets } B \subset U_{\delta, \epsilon}(t)
$$

and

$$
\lambda_{\epsilon}(t, B) \geqslant \sqrt{1+\delta^{2}} \mathcal{L}^{1}(B) \text { for all Borel sets } B \subset U_{\epsilon}(t)-U_{\delta, \epsilon}(t) .
$$

Together with $4.13,4.14$, and the fact that $\sqrt{1+\delta^{2}} \leqslant 3$, this implies

$$
\begin{gathered}
\mathcal{L}^{1}\left(\mathbb{R}-U_{\delta, \epsilon}(t)\right) \stackrel{4.13, \text {, 4.21) }}{\leqslant} \frac{1}{3} \lambda_{\epsilon}\left(t, \mathbb{R}-U_{\epsilon}(t)\right)+\frac{1}{\sqrt{1+\delta^{2}}} \lambda_{\epsilon}\left(t, U_{\epsilon}(t)-U_{\delta, \epsilon}(t)\right) \\
\stackrel{4.14}{\leqslant} \frac{1}{\sqrt{1+\delta^{2}}}\left[\mathcal{L}^{1}\left(\mathbb{R}-U_{\delta, \epsilon}(t)\right)+\epsilon^{2} E_{\epsilon}(t)\right],
\end{gathered}
$$

so that we obtain 4.4):

$$
\mathcal{L}^{1}\left(\mathbb{R}-U_{\delta, \epsilon}(t)\right) \leqslant \frac{1}{\sqrt{1+\delta^{2}}-1} \epsilon^{2} E_{\epsilon}(t) \leqslant C_{0} \frac{\epsilon^{2}}{\delta^{2}} E_{\epsilon}(t) .
$$


We will now estimate $h_{\epsilon}^{\prime}(t)$ in terms of $\lambda_{\epsilon}(t)$ and $E_{\epsilon}(t)$ by the crucial inequality

$$
\int_{\mathbb{R}} h_{\epsilon}(t) \xi^{\prime} \leqslant\left(2 E_{\epsilon}(t) \int_{\mathbb{R}} \xi^{2} \mathrm{~d} \lambda_{\epsilon}(t)\right)^{1 / 2} \quad \text { for all } \xi \in C_{0}^{\infty}(\mathbb{R}) .
$$

Indeed, since $\left(-\frac{\mathrm{d} y_{\epsilon}}{\mathrm{d} s}(t), \frac{\mathrm{d} x_{\epsilon}}{\mathrm{d} s}(t)\right)$ is the outer normal of $\Omega_{\epsilon}(t)$,

$$
\begin{aligned}
\int_{\mathbb{R}} \epsilon h_{\epsilon}(t) \xi^{\prime} & =\int_{\Omega_{\epsilon}(t)} \frac{\mathrm{d} \xi}{\mathrm{d} x}=\int_{\partial \Omega_{\epsilon}(t)}(\xi, 0) \cdot v \\
& =-\int_{-\infty}^{\infty} \xi\left(x_{\epsilon}(t, s)\right) \frac{\mathrm{d} y_{\epsilon}}{\mathrm{d} s}(t, s) \mathrm{d} s \\
& \leqslant\left(\int_{-\infty}^{\infty} \xi\left(x_{\epsilon}(t, s)\right)^{2} \mathrm{~d} s\right)^{1 / 2}\left(\int_{-\infty}^{\infty}\left(\frac{\mathrm{d} y_{\epsilon}}{\mathrm{d} s}(t, s)\right)^{2} \mathrm{~d} s\right)^{1 / 2} \\
& \stackrel{4.10}{\leqslant}\left(\int_{-\infty}^{\infty} \xi\left(x_{\epsilon}(t, s)\right)^{2} \mathrm{~d} s\right)^{1 / 2}\left(2 \int_{-\infty}^{\infty}\left(1-\frac{\mathrm{d} x_{\epsilon}}{\mathrm{d} s}(t, s)\right) \mathrm{d} s\right)^{1 / 2} .
\end{aligned}
$$

According to definition (4.11), the first factor on the right hand side is just

$$
\int_{-\infty}^{\infty} \xi\left(x_{\epsilon}(t, s)\right)^{2} \mathrm{~d} s=\int_{\mathbb{R}} \xi^{2} \mathrm{~d} \lambda_{\epsilon}(t),
$$

whereas according to 4.6 , the second factor is

$$
2 \int_{-\infty}^{\infty}\left(1-\frac{\mathrm{d} x_{\epsilon}}{\mathrm{d} s}(t, s)\right) \mathrm{d} s=2 \epsilon^{2} E_{\epsilon}(t) .
$$

This implies (4.23). The first consequence of 4.23 and 4.20 is 4.3 . A second consequence of (4.23) and (4.14) is

$$
\int_{B}\left|\mathrm{~d} h_{\epsilon}(t)\right| \leqslant\left[2 E_{\epsilon}(t)\left(\mathcal{L}^{1}(B)+\epsilon^{2} E_{\epsilon}(t)\right)\right]^{1 / 2} \text { for all sets } B,
$$

where $\mathrm{d} h_{\epsilon}(t)$ is the measure-valued derivative of $h_{\epsilon}(t)$. Together with 4.22) we obtain (4.5), and the proof is complete.

\section{Convergence of $h_{\epsilon}$}

We introduce the renormalized version $G_{\epsilon}$ of $\Omega_{\epsilon}$,

$$
G_{\epsilon}:=\left\{(t, x, y) \in(0, \infty) \times \mathbb{R} \times(0, \infty):(t, x, \epsilon y) \in \Omega_{\epsilon}\right\},
$$

and the renormalized version $\left(v_{\epsilon}, w_{\epsilon}\right)$ of $u_{\epsilon}$,

$$
\left(v_{\epsilon}(t, x, y), \epsilon w_{\epsilon}(t, x, y)\right):= \begin{cases}u_{\epsilon}(t, x, \epsilon y) & \text { for }(x, y) \in G_{\epsilon}(t), \\ (0,0) & \text { elsewhere. }\end{cases}
$$

The bound (3.4) then translates into

$$
\int_{G_{\epsilon}}\left\{v_{\epsilon}^{2}+\epsilon^{2} w_{\epsilon}^{2}\right\} \quad \text { is bounded for } \epsilon \downarrow 0 .
$$

The goal of this section is the convergence of $h_{\epsilon}$ : 
Proposition 5.1 There exists a non-negative function $h \in C([0, \infty) \times \mathbb{R})$ such that

$$
h_{\epsilon} \stackrel{\epsilon \downarrow 0}{\rightarrow} h \quad \text { uniformly in }[0, T] \times \mathbb{R} \text { for all } T<\infty
$$

for a subsequence. Furthermore

$$
\begin{gathered}
\int_{\mathbb{R}} h(t, x) \mathrm{d} x=1 \quad \text { for all } t \in(0, \infty), \\
h^{\prime} \in L^{\infty}\left((0, \infty) ; L^{2}(\mathbb{R})\right)
\end{gathered}
$$

and for all $0<\delta \leqslant 1$,

$$
\left\{\begin{array}{cc}
h_{\epsilon}^{\prime}(t) & \text { in } U_{\delta, \epsilon}(t) \\
0 & \text { elsewhere }
\end{array}\right\} \stackrel{\epsilon \downarrow 0}{\rightarrow} h^{\prime}(t) \quad \text { in } L^{2}(\mathbb{R}) \text { for all } t \in(0, \infty) .
$$

REMARK 5.2 Proposition 5.1 already proves (2.15) and 2.16 of Theorem 2, and shows that $h$ satisfies (i) of Definition 1 .

The above stated convergence results rely on the boundedness of the following quantities in the limit $\epsilon \downarrow 0$, as stated in Lemma 3.1 .

$$
\begin{gathered}
\int_{\mathbb{R}} h_{\epsilon}(t, x) \mathrm{d} x=M_{\epsilon}(t)=1 \quad \text { for all } t \in(0, \infty), \\
\sup _{t \in(0, \infty)} \mathrm{e}^{-t} \int_{\mathbb{R}} x^{2} h_{\epsilon}(t, x) \mathrm{d} x \leqslant \sup _{t \in(0, \infty)} \mathrm{e}^{-t} Q_{\epsilon}(t) \quad \text { is bounded for } \epsilon \downarrow 0, \\
\sup _{t \in(0, \infty)} E_{\epsilon}(t) \leqslant E_{\epsilon}(0) \quad \text { is bounded for } \epsilon \downarrow 0, \\
\int_{0}^{\infty} D_{\epsilon}(t) \mathrm{d} t \leqslant E_{\epsilon}(0) \quad \text { is bounded for } \epsilon \downarrow 0 .
\end{gathered}
$$

Proof. We fix a $T<\infty$ and use the convention that $C_{0}<\infty$ denotes a generic universal constant, and $C<\infty$ denotes a generic constant only depending on $\sup _{t \in[0, T]} Q_{\epsilon}(t), E_{\epsilon}(0)$ and $T$. The first goal is to derive uniform a priori estimates in Hölder norms for $h_{\epsilon}$, which will entail (5.3).

The bound on $E_{\epsilon}(t)$ translates into a bound on $h_{\epsilon}$ via a combination of the estimates (4.3) and (4.5):

$$
\begin{aligned}
\int_{\mathbb{R}} \xi\left|\mathrm{d} h_{\epsilon}(t)\right| & \leqslant \int_{U_{1, \epsilon}(t)} \xi(x)\left|h_{\epsilon}^{\prime}(t, x)\right| \mathrm{d} x+\int_{\mathbb{R}-U_{1, \epsilon}(t)} \xi\left|\mathrm{d} h_{\epsilon}(t)\right| \\
& \leqslant\left(\int_{\mathbb{R}} \xi(x)^{2} \mathrm{~d} x \int_{U_{1, \epsilon}(t)}\left(h_{\epsilon}^{\prime}(t, x)\right)^{2} \mathrm{~d} x\right)^{1 / 2}+\max _{x \in \mathbb{R}}|\xi(x)|\left|\mathrm{d} h_{\epsilon}(t)\right|\left(\mathbb{R}-U_{1, \epsilon}(t)\right) \\
& \leqslant C_{0}\left(\left(E_{\epsilon}(t) \int_{\mathbb{R}} \xi(x)^{2} \mathrm{~d} x\right)^{1 / 2}+\max _{x \in \mathbb{R}}|\xi(x)| \epsilon E_{\epsilon}(t)\right)
\end{aligned}
$$

for all non-negative Borel functions $\xi$.

The control of $E_{\epsilon}(t)$ and $M_{\epsilon}(t)$ ensures that $h_{\epsilon}$ is uniformly bounded:

$$
h_{\epsilon}(t, x) \leqslant C \quad \text { for all }(t, x) \in[0, \infty) \times \mathbb{R} .
$$


Indeed, for all $x \leqslant x_{0}$ we have

$$
h_{\epsilon}\left(t, x_{0}\right) \leqslant h_{\epsilon}(t, x)+\left|\mathrm{d} h_{\epsilon}(t)\right|\left(\left(x, x_{0}\right)\right),
$$

and integrating in $x$ over $\left(x_{0}-1, x_{0}\right)$ we obtain

$$
\begin{aligned}
h_{\epsilon}\left(t, x_{0}\right) & \leqslant \int_{x_{0}-1}^{x_{0}} h_{\epsilon}(t, x) \mathrm{d} x+\int_{x_{0}-1}^{x_{0}}\left|\mathrm{~d} h_{\epsilon}(t)\right|\left(\left(x, x_{0}\right)\right) \mathrm{d} x \\
& \leqslant \int_{\mathbb{R}} h_{\epsilon}(t, x) \mathrm{d} x+\left|\mathrm{d} h_{\epsilon}(t)\right|\left(\left(x_{0}-1, x_{0}\right)\right) \\
& \stackrel{5.11}{\leqslant} M_{\epsilon}(t)+C_{0}\left(E_{\epsilon}(t)^{1 / 2}+\epsilon E_{\epsilon}(t)\right) \leqslant C .
\end{aligned}
$$

The control of $E_{\epsilon}(t)$ and $Q_{\epsilon}(t)$ ensures that $h_{\epsilon}$ is uniformly small for $|x| \gg 1$ :

$$
h_{\epsilon}(t, x) \leqslant C \frac{1+\epsilon|x|^{1 / 2}}{|x|^{1 / 2}}, \quad|x|>1 .
$$

In order to see (5.13), by symmetry we fix an $x_{0}>1$ and consider the interval $I=\left(x_{0}, x_{0}+1 / x_{0}\right)$. By a standard interpolation result, for any function $h$ we have

$$
\sup _{I}|h| \leqslant\left(\frac{1}{\mathcal{L}^{1}(I)} \int_{I}|h|+\int_{I}|\mathrm{~d} h|\right) .
$$

This yields

$$
\begin{aligned}
h_{\epsilon}\left(t, x_{0}\right) \leqslant \sup _{x \in I} h_{\epsilon}(t, x) & \leqslant C\left(\left|x_{0}\right| \int_{I} h_{\epsilon}(t, x) \mathrm{d} x+\int_{I}\left|\mathrm{~d} h_{\epsilon}(t)\right|\right) \\
& \stackrel{5.11}{\leqslant} C\left(\frac{1}{\left|x_{0}\right|} \int_{I} h_{\epsilon}(t, x) x^{2} \mathrm{~d} x+\frac{1}{\left|x_{0}\right|^{1 / 2}}+\epsilon\right),
\end{aligned}
$$

and using (5.8) we obtain 5.13). Now (5.12) and (5.13) combine into

$$
h_{\epsilon}(t, x) \leqslant C \frac{1+\epsilon|x|^{1 / 2}}{1+|x|^{1 / 2}} \quad \text { for all }(t, x) \in[0, T] \times \mathbb{R} .
$$

The control of $E_{\epsilon}(t)$ enables us to show that $h_{\epsilon}$ satisfies the following uniform continuity property in $x$ :

$$
\left|h_{\epsilon}\left(t_{0}, x_{1}\right)-h_{\epsilon}\left(t_{0}, x_{2}\right)\right| \leqslant C\left(\left|x_{1}-x_{2}\right|^{1 / 2}+\epsilon\right)
$$

for all $\left(t_{0}, x_{1}\right),\left(t_{0}, x_{2}\right) \in[0, \infty) \times \mathbb{R}$. Indeed, we have

$$
\begin{aligned}
\left|h_{\epsilon}\left(t_{0}, x_{1}\right)-h_{\epsilon}\left(t_{0}, x_{2}\right)\right| & \leqslant\left|\mathrm{d} h_{\epsilon}\left(t_{0}\right)\right|\left(\left(x_{1}, x_{2}\right)\right) \\
& \stackrel{5.11}{\leqslant} C_{0}\left(\left(\left|x_{1}-x_{2}\right| E_{\epsilon}(t)\right)^{1 / 2}+\epsilon E_{\epsilon}(t)\right) \\
& \leqslant C\left(\left|x_{1}-x_{2}\right|^{1 / 2}+\epsilon\right) .
\end{aligned}
$$

The control of $D_{\epsilon}(t)$ and 5.12 enable us to show that spatial averages of $h_{\epsilon}$ are uniformly continuous in $t$ :

$$
\left|\int_{\mathbb{R}} h_{\epsilon}\left(t_{1}, x\right) \varphi(x) \mathrm{d} x-\int_{\mathbb{R}} h_{\epsilon}\left(t_{2}, x\right) \varphi(x) \mathrm{d} x\right| \leqslant C\left(\left|t_{1}-t_{2}\right| \int_{\mathbb{R}}\left(\varphi^{\prime}(x)\right)^{2} \mathrm{~d} x\right)^{1 / 2}
$$


for all $t_{1}, t_{2} \in[0, \infty)$ and $\varphi \in C_{0}^{\infty}(\mathbb{R})$. Indeed, since

$$
\begin{aligned}
\frac{\mathrm{d}}{\mathrm{d} t} \int_{\mathbb{R}} h_{\epsilon}(t, x) \varphi(x) \mathrm{d} x & =\frac{\mathrm{d}}{\mathrm{d} t} \int_{\Omega_{\epsilon}(t)} \frac{1}{\epsilon} \varphi(x) \mathrm{d} x \mathrm{~d} y=\int_{\partial \Omega_{\epsilon}(t)} \frac{1}{\epsilon} V \cdot \varphi(x) \mathrm{d} x \mathrm{~d} y \\
& \stackrel{3.8}{=} \int_{\Omega_{\epsilon}(t)} u \cdot \nabla \varphi(x) \mathrm{d} x \mathrm{~d} y=\int_{G_{\epsilon}(t)} v_{\epsilon}(t, x, y) \varphi^{\prime}(x) \mathrm{d} x \mathrm{~d} y,
\end{aligned}
$$

we obtain

$$
\begin{aligned}
& \left|\int_{\mathbb{R}} h_{\epsilon}\left(t_{1}, x\right) \varphi(x) \mathrm{d} x-\int_{\mathbb{R}} h_{\epsilon}\left(t_{2}, x\right) \varphi(x) \mathrm{d} x\right| \\
& \quad \leqslant\left(\int_{G_{\epsilon}} v_{\epsilon}(t, x, y)^{2} \mathrm{~d} t \mathrm{~d} x \mathrm{~d} y\right)^{1 / 2}\left(\int_{G_{\epsilon} \cap\left(\left(t_{1}, t_{2}\right) \times \mathbb{R} \times(0, \infty)\right)} \varphi^{\prime}(x)^{2} \mathrm{~d} t \mathrm{~d} x \mathrm{~d} y\right)^{1 / 2} \\
& \stackrel{5.10}{\leqslant} C\left(\left|t_{1}-t_{2}\right| \max _{\left(t_{1}, t_{2}\right) \times \mathbb{R}} h_{\epsilon} \int_{\mathbb{R}} \varphi^{\prime}(x)^{2} \mathrm{~d} x\right)^{1 / 2},
\end{aligned}
$$

and 5.12) implies 5.16.

Interpolating the strong uniform control of continuity in $x$ given through 5.15 and the weak uniform control of continuity in $t$ given through (5.16), we obtain the following uniform continuity property in $t$ :

$$
\left|h_{\epsilon}\left(t_{1}, x_{0}\right)-h_{\epsilon}\left(t_{2}, x_{0}\right)\right| \leqslant C\left(\left|t_{1}-t_{2}\right|^{1 / 8}+\epsilon\right)
$$

for all $\left(t_{1}, x_{0}\right),\left(t_{2}, x_{0}\right) \in[0, \infty) \times \mathbb{R}$. Indeed, fix a smooth $\varphi$ with support in $(-1,1)$ and unit integral. We rescale $\varphi$ by a $\delta>0$ to be chosen later:

$$
\varphi_{\delta}(x)=\frac{1}{\delta} \varphi\left(\frac{x}{\delta}\right)
$$

We have

$$
\begin{aligned}
\mid h_{\epsilon}\left(t_{1}, x_{0}\right) & -h_{\epsilon}\left(t_{2}, x_{0}\right) \mid \\
\leqslant & \int_{\mathbb{R}} \varphi_{\delta}\left(x-x_{0}\right)\left|h_{\epsilon}\left(t_{1}, x\right)-h_{\epsilon}\left(t_{1}, x_{0}\right)\right| \mathrm{d} x \\
+ & \left|\int_{\mathbb{R}} \varphi_{\delta}\left(x-x_{0}\right) h_{\epsilon}\left(t_{1}, x\right) \mathrm{d} x-\int_{\mathbb{R}} \varphi_{\delta}\left(x-x_{0}\right) h_{\epsilon}\left(t_{2}, x\right) \mathrm{d} x\right| \\
& +\int_{\mathbb{R}} \varphi_{\delta}\left(x-x_{0}\right)\left|h_{\epsilon}\left(t_{2}, x\right)-h_{\epsilon}\left(t_{2}, x_{0}\right)\right| \mathrm{d} x \\
\qquad & C\left(\int_{\mathbb{R}} \varphi_{\delta}\left(x-x_{0}\right)\left|x-x_{0}\right|^{1 / 2} \mathrm{~d} x+\epsilon\right)+C\left(\left|t_{1}-t_{2}\right| \int_{\mathbb{R}}\left(\varphi_{\delta}^{\prime}\left(x-x_{0}\right)\right)^{2} \mathrm{~d} x\right)^{1 / 2} \\
\leqslant & C\left(\delta+\frac{1}{\delta^{3}}\left|t_{1}-t_{2}\right|\right)^{1 / 2}+C \epsilon .
\end{aligned}
$$

This turns into 5.17 for $\delta=\left|t_{1}-t_{2}\right|^{1 / 4}$.

By a slight generalization of the Arzelà-Ascoli argument, 5.12, (5.15) and 5.17) imply the existence of a continuous function $h$ on $[0, \infty) \times \mathbb{R}$ such that

$$
h_{\epsilon} \stackrel{\epsilon \downarrow 0}{\rightarrow} h \quad \text { locally uniformly in }[0, \infty) \times \mathbb{R}
$$


for a subsequence. Taking now (5.14) into account, we see that this improves to the global uniform convergence stated in (5.3). Thanks to the bound (5.8), (5.7) is preserved under the convergence (5.3). This yields (5.4).

The last task is to establish 5.5 and 5.6 . Fix a $t \in[0, T]$ and a $\delta \in(0,1]$. For any test function $\xi \in C_{0}^{\infty}(\mathbb{R})$, we may write

$$
\begin{aligned}
-\int_{\mathbb{R}} h_{\epsilon}(t) \xi^{\prime} & =\int_{U_{\delta, \epsilon}(t)} h_{\epsilon}^{\prime}(t) \xi+\int_{\mathbb{R}-U_{\delta, \epsilon}(t)} \xi \mathrm{d} h_{\epsilon}(t) \\
& \left(2 \sqrt{1+\delta^{2}} E_{\epsilon}(t) \int_{\mathbb{R}} \xi^{2}\right)^{1 / 2}+C_{0} \frac{\epsilon}{\delta} E_{\epsilon}(t) \max _{\mathbb{R}}|\xi|,
\end{aligned}
$$

which (since $\left.E_{\epsilon}(t) \leqslant E_{\epsilon}(0)\right)$ in the limit $\epsilon \downarrow 0$ turns into

$$
-\int_{\mathbb{R}} h(t) \xi^{\prime} \leqslant\left(C \sqrt{1+\delta^{2}} \int_{\mathbb{R}} \xi^{2}\right)^{1 / 2} .
$$

Since $\xi$ and $\delta$ were arbitrary, this implies 5.5 . Therefore, we gather from the representation 5.18 and $(4.5)$ that

$$
\int_{\mathbb{R}} h^{\prime}(t) \xi=\lim _{\epsilon \downarrow 0} \int_{U_{\delta, \epsilon}(t)} h_{\epsilon}^{\prime}(t) \xi .
$$

According to 4.3 we have

$$
\int_{U_{\delta, \epsilon}(t)} h_{\epsilon}^{\prime}(t)^{2} \leqslant C
$$

Now the distributional convergence 5.19 together with the uniform $L^{2}$-bound in 5.20 imply the weak $L^{2}$-convergence 5.6, and the proof is complete.

\section{Convergence of $v_{\epsilon}$ and $\hat{\pi}_{\epsilon}$}

The renormalized pressure

$$
\pi_{\epsilon}(t, x, y):=p_{\epsilon}(t, x, \epsilon y) \quad \text { for }(t, x, y) \in G_{\epsilon}
$$

is such that Darcy's law 3.7 turns into

$$
v_{\epsilon}=-\pi_{\epsilon}^{\prime} \quad \text { and } \quad \epsilon^{2} w_{\epsilon}=-\frac{\partial \pi_{\epsilon}}{\partial y} \quad \text { in } G_{\epsilon} .
$$

Therefore, the bound $[5.2]$ translates into

$$
\int_{G_{\epsilon}}\left\{\left(\pi_{\epsilon}^{\prime}\right)^{2}+\frac{1}{\epsilon^{2}}\left(\frac{\partial \pi_{\epsilon}}{\partial y}\right)^{2}\right\} \quad \text { is bounded for } \epsilon \downarrow 0 .
$$

We also observe that the weak formulation of the kinetic conditions $3.8-3.10$ is

$$
\int_{\Omega_{\epsilon}}\left\{\partial_{t} \zeta+u_{\epsilon} \cdot \nabla \zeta\right\}=0 \quad \text { for all } \zeta \in C_{0}^{\infty}((0, \infty) \times \mathbb{R} \times \mathbb{R}) .
$$


For test functions $\zeta$ independent of $y$, this translates on the renormalized level into the continuity equation

$$
\int_{(0, \infty) \times \mathbb{R}} h_{\epsilon}(t, x) \partial_{t} \zeta(t, x) \mathrm{d} t \mathrm{~d} x+\int_{(0, \infty) \times \mathbb{R} \times(0, \infty)} v_{\epsilon}(t, x, y) \zeta^{\prime}(t, x) \mathrm{d} t \mathrm{~d} x \mathrm{~d} y=0
$$

for all $\zeta \in C_{0}^{\infty}((0, \infty) \times \mathbb{R})$. Let

$$
G:=\{(t, x, y): t>0,0<y<h(t, x)\} .
$$

In view of 6.3, one expects that the pressure-and hence the velocity-becomes $y$-independent in the limit $\epsilon \downarrow 0$. But the convergence of $\pi_{\epsilon}$ is delicate: We only control its gradient on the $\epsilon$ dependent domain $G_{\epsilon}$ which may pinch off in the limit. Hence, in order to apply a Poincaré estimate, we have to consider connected components of the limit domain $G$ and stay safely in the interior of $\{h>0\} \times(0, \infty)$. More precisely, let $J$ and $I$ be open bounded intervals with $\bar{J} \times \bar{I} \subset\{h>0\}$. According to (5.3) and 4.1, there exists an $h_{0}>0$ such that

$$
G_{\epsilon} \supset \bar{J} \times \bar{I} \times\left(0, h_{0}\right] \quad \text { for } \epsilon \ll 1 .
$$

Furthermore, we have to cut away the "overhanging" parts of $G_{\epsilon}$ by introducing

$$
\underline{G}_{\epsilon}:=\left\{(t, x, y):(t, x, \tilde{y}) \in G_{\epsilon} \text { for all } 0<\tilde{y}<y\right\} .
$$

With $\underline{G}_{\epsilon} \cap(J \times I \times(0, \infty))$, we now have a domain on which the Poincaré estimate does not deteriorate for $\epsilon \downarrow 0$. Hence we expect to control a "relative" pressure

$$
\begin{aligned}
& \hat{\pi}_{\epsilon}(t, x, y) \\
& \quad:= \begin{cases}\pi_{\epsilon}(t, x, y)-f_{I \times\left(0, h_{0}\right)} \pi_{\epsilon}(t) \text { for }(t, x, y) \in \underline{G}_{\epsilon} & \text { for }(t, x, y) \in J \times I \times(0, \infty) \\
0 & \text { elsewhere }\end{cases}
\end{aligned}
$$

in $L^{2}$ and hence obtain a weak limit $\hat{\pi}$, as stated in Proposition 6.1 (i). It is immediate that $\hat{\pi}$ does not depend on $y$ (see Proposition 6.1(ii)). Ultimately, we will need the convergence of the trace of $\hat{\pi}_{\epsilon}$ on the horizontal plane and on vertical slices (Proposition 6.1 (iii) and (iv)):

Proposition 6.1 Let $J \times I \subset(0, \infty) \times \mathbb{R}$ be an open bounded rectangle such that $h>0$ in its closure, and let $\hat{\pi}_{\epsilon}$ be defined by 6.6. Then:

(i) There exists a $\hat{\pi} \in L^{2}(J \times I \times(0, \infty))$ such that for a subsequence

$$
\hat{\pi}_{\epsilon} \rightarrow \hat{\pi} \quad \text { in } L^{2}(J \times I \times(0, \infty)) .
$$

(ii) There exists a $\hat{\pi} \in L^{2}(J \times I)$ (we use the same notation) such that

$$
\hat{\pi}(t, x, y)=\left\{\begin{array}{ll}
\hat{\pi}(t, x) & \text { for } 0<y<h(t, x) \\
0 & \text { elsewhere }
\end{array}\right. \text { a.e. }
$$

(iii) $\hat{\pi}_{\epsilon}(\cdot, \cdot, 0) \rightarrow \hat{\pi}$ in $L^{2}(J \times I)$. 
(iv) For any $p \in[1,2)$ and a.e. $c \in I$,

$$
\hat{\pi}_{\epsilon}(\cdot, c, \cdot) \rightarrow\left\{\begin{array}{ll}
\hat{\pi}(t, c) & \text { for } 0<y<h(t, c) \\
0 & \text { elsewhere }
\end{array} \text { in } L^{p}(J \times(0, \infty)) .\right.
$$

As opposed to $\pi_{\epsilon}$, the weak convergence of $v_{\epsilon}$ comes almost for free from (5.2). Having established the weak convergence of $\hat{\pi}_{\epsilon}$ in Proposition 6.1. from 6.4 and 6.2 we easily recover the limiting continuity equation and Darcy's law, respectively.

Proposition 6.2 (i) There exists a $v \in L^{2}((0, \infty) \times \mathbb{R} \times(0, \infty))$ such that for a subsequence

$$
v_{\epsilon} \rightarrow v \quad \text { in } L^{2}((0, \infty) \times \mathbb{R} \times(0, \infty)) .
$$

(ii) There exists a measurable $v:(0, \infty) \times \mathbb{R} \rightarrow \mathbb{R}$ such that

$$
v(t, x, y)=\left\{\begin{array}{ll}
v(t, x) & \text { for } 0<y<h(t, x) \\
0 & \text { elsewhere }
\end{array}\right. \text { a.e. }
$$

In particular

$$
\int_{(0, \infty) \times \mathbb{R}} h v^{2}<\infty
$$

(iii)

$$
\partial_{t} h+(h v)^{\prime}=0 \quad \text { distributionally in }(0, \infty) \times \mathbb{R} .
$$

(iv) Let $J, I, \hat{\pi}$ be as in Proposition 6.1 Then

$$
-\hat{\pi}^{\prime}=v \quad \text { distributionally in } J \times I .
$$

REMARK 6.3 In view of 6.9 and 6.11, in fact $\hat{\pi} \in L^{2}\left(J ; H^{1}(I)\right)$. As a consequence, $\hat{\pi}(t) \in$ $C_{\text {loc }}(\{h(t)>0\})$ for almost every $t \in(0, \infty)$.

Proof of Proposition 6.1 Let $C<\infty$ denote a generic constant independent of $\epsilon$. We start by showing that

$$
\int_{J \times I \times(0, \infty)}\left|\hat{\pi}_{\epsilon}\right|^{2} \leqslant C
$$

Since by definition 6.6 the average of $\hat{\pi}_{\epsilon}(t)$ vanishes on $I \times\left(0, h_{0}\right)$ for all $t \in J$, we obtain by the Poincaré estimate on the bounded square $I \times\left(0, h_{0}\right)$,

$$
\int_{I \times\left(0, h_{0}\right)}\left|\hat{\pi}_{\epsilon}(t)\right|^{2} \leqslant C \int_{I \times\left(0, h_{0}\right)}\left\{\left|\hat{\pi}_{\epsilon}^{\prime}(t)\right|^{2}+\left|\frac{\partial \hat{\pi}_{\epsilon}}{\partial y}(t)\right|^{2}\right\} .
$$

Integrating over $t \in J$, we get

$$
\int_{J \times I \times\left(0, h_{0}\right)}\left|\hat{\pi}_{\epsilon}\right|^{2} \leqslant C \int_{G_{\epsilon}}\left\{\left|\pi_{\epsilon}^{\prime}\right|^{2}+\left|\frac{\partial \pi_{\epsilon}}{\partial y}\right|^{2}\right\} .
$$

We now use the Poincaré estimate on

$$
\underline{G}_{\epsilon}(t, x):=\left\{y \in(0, \infty):(t, x, y) \in \underline{G}_{\epsilon}\right\},
$$


which thanks to 6.5 are intervals (and uniformly bounded by (5.3) and (4.1), and thus obtain

$$
\int_{\underline{G}_{\epsilon}(t, x)}\left|\hat{\pi}_{\epsilon}(t, x)\right|^{2} \leqslant C\left\{\int_{\left(0, h_{0}\right)}\left|\hat{\pi}_{\epsilon}(t, x)\right|^{2}+\int_{\underline{G}_{\epsilon}(t, x)}\left|\frac{\partial \hat{\pi}_{\epsilon}}{\partial y}(t, x)\right|^{2}\right\} .
$$

Integrating over $(t, x) \in J \times I$ and recalling that $\hat{\pi}_{\epsilon}=0$ outside of $\underline{G}_{\epsilon}$, we get

$$
\int_{J \times I \times(0, \infty)}\left|\hat{\pi}_{\epsilon}\right|^{2} \leqslant C\left\{\int_{J \times I \times\left(0, h_{0}\right)}\left|\hat{\pi}_{\epsilon}\right|^{2}+\int_{G_{\epsilon}}\left|\frac{\partial \pi_{\epsilon}}{\partial y}\right|^{2}\right\} .
$$

Combining 6.13 and 6.14, we gather

$$
\int_{J \times I \times(0, \infty)}\left|\hat{\pi}_{\epsilon}\right|^{2} \leqslant C \int_{G_{\epsilon}}\left\{\left|\pi_{\epsilon}^{\prime}\right|^{2}+\left|\frac{\partial \pi_{\epsilon}}{\partial y}\right|^{2}\right\} \stackrel{6.3}{\leqslant} C .
$$

This establishes 6.12 and thus part (i) of Proposition 6.1

By definition

$$
\hat{\pi}_{\epsilon}=0 \quad \text { outside of }(J \times I \times(0, \infty)) \cap \underline{G}_{\epsilon},
$$

which because of (5.3) and (4.1), and the convergence stated in part (i), yields

$$
\hat{\pi}=0 \quad \text { outside of }(J \times I \times(0, \infty)) \cap G .
$$

On the other hand, 6.3 implies in particular

$$
\int_{G_{\epsilon}}\left|\frac{\partial \hat{\pi}_{\epsilon}}{\partial y}\right|^{2} \stackrel{\epsilon \downarrow 0}{\rightarrow} 0 .
$$

Once again, because of 5.3 and (4.1), this translates into

$$
\frac{\partial \hat{\pi}}{\partial y}=0 \quad \text { distributionally in }(J \times I \times(0, \infty)) \cap G
$$

under the convergence stated in part (i). Now part (i) and (6.16) yield part (ii).

In order to improve the weak convergence stated in part (i) to the weak convergence of the $\{y=0\}$-trace stated in part (iii), it is sufficient to show the following uniform continuity property:

$$
\lim _{y \downarrow 0} \limsup _{\epsilon \downarrow 0} \int_{J \times I}\left|\hat{\pi}_{\epsilon}(t, x, y)-\hat{\pi}_{\epsilon}(t, x, 0)\right|^{2} \mathrm{~d} t \mathrm{~d} x=0 .
$$

This easily follows from the fact that for $y \in\left(0, h_{0}\right)$,

$$
\int_{J \times I}\left|\hat{\pi}_{\epsilon}(t, x, y)-\hat{\pi}_{\epsilon}(t, x, 0)\right|^{2} \mathrm{~d} t \mathrm{~d} x \leqslant|y| \int_{J \times I \times(0, y)}\left|\frac{\partial \hat{\pi}_{\epsilon}}{\partial y}\right|^{2} \stackrel{6.3}{\leqslant} C \epsilon^{2}\left|h_{0}\right| .
$$

In order to improve the weak convergence stated in part (i) to the weak convergence of almost every $\{x=c\}$-trace stated in part (iv), it is sufficient to show strong convergence of the $(t, y)$ averages:

$$
\int_{J \times(0, \infty)} \hat{\pi}_{\epsilon}(t, \cdot, y) \zeta(t, y) \mathrm{d} t \mathrm{~d} y \rightarrow \int_{J \times(0, \infty)} \hat{\pi}(t, \cdot, y) \zeta(t, y) \mathrm{d} t \mathrm{~d} y \quad \text { in } L_{\mathrm{loc}}^{p}(I)
$$


for an arbitrary $\zeta \in L^{p^{\prime}}(J \times(0, \infty))$, with $p \in[1,2)$ and $p^{\prime}=p /(p-1)$. This follows from a uniform continuity property similar to 6.177 , namely

$$
\lim _{\tau \rightarrow 0} \limsup _{\epsilon \downarrow 0} \int_{J \times K \times(0, \infty)}\left|\hat{\pi}_{\epsilon}(t, x-\tau, y)-\hat{\pi}_{\epsilon}(t, x, y)\right|^{p} \mathrm{~d} t \mathrm{~d} x \mathrm{~d} y=0
$$

for any compact $K \subset I$. In fact, we will show for $|\tau| \ll 1$,

$$
\limsup _{\epsilon \downarrow 0} \int_{J \times K \times(0, \infty)}\left|\hat{\pi}_{\epsilon}^{(\tau)}-\hat{\pi}_{\epsilon}\right|^{p} \leqslant C|\tau|^{(2-p) / 4} ;
$$

here and in what follows $C$ is a generic constant independent of $\epsilon$ and $\tau$, and the superscript $(\tau)$ stands for translation in $x$-direction.

In order to prove (6.18) it is enough to consider the case $\tau>0$, as the case $\tau<0$ is analogous. We start by observing that

$$
\sup _{t \in(0, \infty), x_{1} \neq x_{2}} \frac{\left|h\left(t, x_{1}\right)-h\left(t, x_{2}\right)\right|}{\left|x_{1}-x_{2}\right|^{1 / 2}} \leqslant C \sup _{t \in(0, \infty)} \int_{\mathbb{R}}\left|h^{\prime}(t)\right|^{2} \stackrel{5.5}{\leqslant} C .
$$

We introduce the notation

$$
G^{(-\delta)}:=\{(t, x, y) \in(0, \infty) \times \mathbb{R} \times(0, \infty): 0<y<h(t, x)-\delta\}
$$

for a downward shift, and

$$
G^{(-\delta, \tau)}:=\left\{(t, x, y) \in(0, \infty) \times \mathbb{R} \times(0, \infty):(t, x-\sigma, y) \in G^{(-\delta)} \forall \sigma \in(0, \tau)\right\}
$$

for simultaneous sidewards reduction. According to $\sqrt{5.3}$ and 4.1 , we thus obtain for any $\tau>0$,

$$
H^{\tau}:=G^{(-C \sqrt{\tau}, \tau)} \subset G^{(-C \sqrt{\tau})} \subset G_{\epsilon} \quad \text { provided } \epsilon \ll 1 .
$$

On the other hand, in view of (6.19),

$$
G^{(-2 C \sqrt{\tau})} \subseteq H^{\tau} .
$$

We now split the integral $(6.18)$ into two parts:

$$
\int_{J \times K \times(0, \infty)}\left|\hat{\pi}_{\epsilon}^{(\tau)}-\hat{\pi}_{\epsilon}\right|^{p} \leqslant \int_{(J \times K \times(0, \infty)) \cap H^{\tau}}\left|\hat{\pi}_{\epsilon}^{(\tau)}-\hat{\pi}_{\epsilon}\right|^{p}+\int_{(J \times K \times(0, \infty))-H^{\tau}}\left|\hat{\pi}_{\epsilon}^{(\tau)}-\hat{\pi}_{\epsilon}\right|^{p} .
$$

On the first part, because of definitions 6.20 and 6.21 , we may represent the difference $\hat{\pi}_{\epsilon}^{(\tau)}-\hat{\pi}_{\epsilon}$ via $\pi_{\epsilon}^{\prime}$; provided $\tau$ is so small that $K_{\tau}:=K+B(0, \tau) \subset I$, we obtain

$$
\begin{aligned}
\int_{(J \times K \times(0, \infty)) \cap H^{\tau}}\left|\hat{\pi}_{\epsilon}^{(\tau)}-\hat{\pi}_{\epsilon}\right|^{p} & \leqslant \tau^{p} \int_{\left(J \times K_{\tau} \times(0, \infty)\right) \cap H^{\tau}}\left|\pi_{\epsilon}^{\prime}\right|^{p} \\
& \stackrel{6.21]}{\leqslant} \tau^{p}\left(\int_{J \times \mathbb{R}} h_{\epsilon}\right)^{(2-p) / 2}\left(\int_{G_{\epsilon}}\left|\pi_{\epsilon}^{\prime}\right|^{2}\right)^{p / 2} \\
& \stackrel{5.45}{=} \tau^{p}\left(\mathcal{L}^{1}(J)\right)^{(2-p) / 2}\left(\int_{G_{\epsilon}}\left|\pi_{\epsilon}^{\prime}\right|^{2}\right)^{p / 2} \\
& \stackrel{6.3}{\leqslant} C \tau^{p} .
\end{aligned}
$$


For the second term, we observe that, with $K^{(-\tau)}=K-\tau$,

$$
\begin{aligned}
& \int_{(J \times K \times(0, \infty))-H^{\tau}}\left|\hat{\pi}_{\epsilon}^{(\tau)}-\hat{\pi}_{\epsilon}\right|^{p} \leqslant \int_{(J \times K \times(0, \infty))-H^{\tau}}\left|\hat{\pi}_{\epsilon}\right|^{p}+\int_{(J \times K \times(0, \infty))-H^{\tau}}\left|\hat{\pi}_{\epsilon}^{(\tau)}\right|^{p} \\
& \underset{5.5,6.22}{\leqslant}\left(\int_{J \times K}\left(h_{\epsilon}-(h-2 C \sqrt{\tau})\right)\right)^{(2-p) / 2}\left(\int_{\underline{G}_{\epsilon} \cap(J \times I \times(0, \infty))}\left|\hat{\pi}_{\epsilon}\right|^{2}\right)^{p / 2} \\
& +\left(\int_{J \times K^{(-\tau)}}\left(h_{\epsilon}-(h-2 C \sqrt{\tau})\right)\right)^{(2-p) / 2}\left(\int_{\underline{G}_{\epsilon} \cap(J \times I \times(0, \infty))}\left|\hat{\pi}_{\epsilon}\right|^{2}\right)^{p / 2} \\
& \stackrel{6.12}{\leqslant} 2 C\left(\int_{J \times K_{\tau}}\left(h_{\epsilon}-(h-2 C \sqrt{\tau})\right)\right)^{(2-p) / 2} \text {. }
\end{aligned}
$$

Hence

$$
\limsup _{\epsilon \downarrow 0} \int_{(J \times K \times(0, \infty))-H^{\tau}}\left|\hat{\pi}_{\epsilon}^{(\tau)}-\hat{\pi}_{\epsilon}\right|^{p} \leqslant C\left(\int_{J \times K_{\tau}} \sqrt{\tau}\right)^{(2-p) / 2} \leqslant C \tau^{(2-p) / 4}
$$

and the proof is complete.

\section{Horizontal and vertical variations}

This section is the core of our argument. We test the $\epsilon$-equation with two distinct classes of fields $\tilde{u}$. Velocity fields $\tilde{u}$ of the first class are vertical and turn into variations of the dependent argument, i.e. $h$, in the limit $\epsilon \downarrow 0$ (see Fig. 2). We use the convexity of the total length of $\operatorname{graph}(h)$ in terms of $h$ to transform the identity into an inequality, which is more robust under the passage to the limit. This inequality is stated in Lemma 7.1

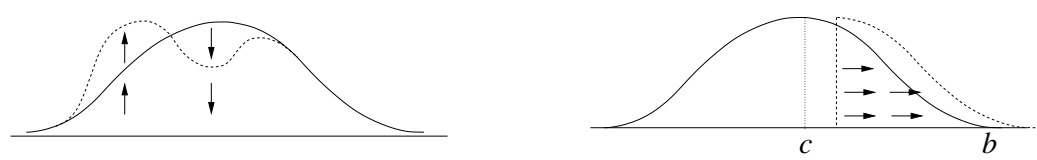

FIG. 2. Typical vertical and horizontal variations.

The zero contact-angle condition is a local equilibrium condition under (horizontal) translations of the contact point. The vertical variations are limited to the interior of $\{h>0\}$ and hence are oblivious to the zero contact-angle condition. Velocity fields $\tilde{u}$ of the second class are horizontal and turn into translations of a contact point $b$, i.e. $b \in \partial\{h>0\}$, in the limit (see Fig. 2). It is this class of variations which encodes the zero contact-angle condition in a way sufficiently robust for the passage to the limit. The relevant inequality is stated in Lemma 7.2

In the first lemma, we wish to take test fields $\tilde{u}$ of the form

$$
\tilde{u}(x, y)=(0, \tilde{w}(x)),
$$

with $\operatorname{supp}(w) \subset\{h>0\}$. Unfortunately, a technical difficulty arises: The boundary curve is not a graph over the entire $x$-axis. Hence, in order to use convexity, $w$ has to be constant over the bad portions of the $x$-axis. 
LEMmA 7.1 Let $J \times I \subset(0, \infty) \times \mathbb{R}$ be an open bounded rectangle such that $h>0$ in its closure. Then

$$
\begin{aligned}
\int_{U_{\delta, \epsilon} \cap(J \times I)} \frac{1}{\epsilon^{2}}\left(\sqrt{1+\left(\epsilon \tilde{h}_{\epsilon}^{\prime}\right)^{2}}-1\right)-\int_{U_{\delta, \epsilon} \cap(J \times I)} \frac{1}{\epsilon^{2}}\left(\sqrt{1+\left(\epsilon h_{\epsilon}^{\prime}\right)^{2}}-1\right) \\
\geqslant \int_{G_{\epsilon} \cap(J \times I)}-\epsilon^{2} w_{\epsilon} \tilde{w}_{\epsilon}+\int_{J \times I \times\{0\}} \pi_{\epsilon} \tilde{w}_{\epsilon}
\end{aligned}
$$

for all $0<\delta \leqslant 1$ and for all $\tilde{w}_{\epsilon} \in L^{2}\left(J ; H^{1}(\mathbb{R})\right)$ with

$$
\begin{aligned}
\tilde{h}_{\epsilon} & :=h_{\epsilon}+\tilde{w}_{\epsilon}, \\
\tilde{w}_{\epsilon}(t) & =0 \quad \text { outside of } I \text { for a.e. } t \in J, \\
\tilde{w}_{\epsilon}^{\prime}(t) & =0 \quad \text { outside of } U_{\delta, \epsilon}(t) \text { for a.e. } t \in J .
\end{aligned}
$$

In the second lemma, we wish to take test fields of the form

$$
\tilde{u}(x, y)= \begin{cases}(1,0) & \text { for } c<x<b, \\ (0,0) & \text { elsewhere, }\end{cases}
$$

where $c \in\{h>0\}$ and $b \in \partial\{h>0\}$ (evoking symmetry, we will hereafter only consider right end-points of connected components of $\{h>0\}$ ). Unfortunately, a technical difficulty arises with these test fields: There is not necessarily an $\epsilon$-contact point $b_{\epsilon}$ near a contact point $b$ of the limit. But there is always a point $\left(x_{\epsilon}^{-}, y_{\epsilon}^{-}\right)$on the boundary curve (cf. Fig. 3) with horizontal tangent and $y_{\epsilon}^{-}=o(1)$, such that the area between $b$ and $x_{\epsilon}^{-}$is $o(1)$. This will be sufficient for our purpose.

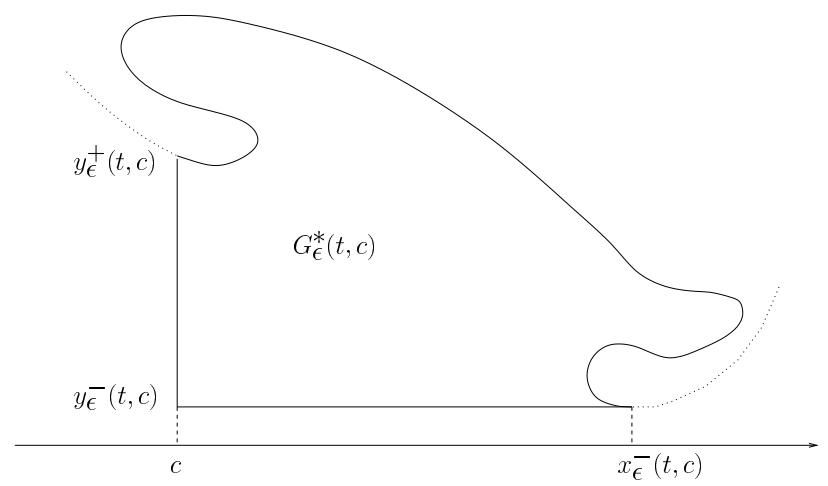

FIG. 3. The set $G_{\epsilon}^{*}(t, c)$ in Lemma 7.2

Lemma 7.2 Let $J \times I \subset(0, \infty) \times \mathbb{R}$ be an open rectangle such that $h>0$ in its closure, $c \in I$, and let

$$
\begin{aligned}
b(t, c) & :=\sup \{x>c: h(t, \xi)>0 \forall \xi \in(c, x)\}, \\
G_{\epsilon}^{(c)}(t) & :=G_{\epsilon}(t) \cap\{c \leqslant x \leqslant b(t, c)\} .
\end{aligned}
$$

There exist a set $G_{\epsilon}^{*}(t, c) \subset G_{\epsilon}(t)$ which is close to $G_{\epsilon}^{(c)}(t)$ in the sense that

$$
\lim _{\epsilon \downarrow 0} \mathcal{L}^{2}\left(G_{\epsilon}^{*}(t, c) \triangle G_{\epsilon}^{(c)}(t)\right)=0 \quad \forall t \in J,
$$


and an interval $\left(y_{\epsilon}^{-}(t, c), y_{\epsilon}^{+}(t, c)\right)$ which is close to $(0, h(t, c))$ in the sense that

$$
\lim _{\epsilon \downarrow 0} y_{\epsilon}^{-}(t, c)=0, \quad \lim _{\epsilon \downarrow 0} y_{\epsilon}^{+}(t, c)=h(t, c) \quad \text { uniformly for } t \in J,
$$

such that

$$
\int_{J} \int_{G_{\epsilon}^{*}(t, c)} v_{\epsilon}(t) \mathrm{d} t \geqslant \int_{J} \int_{y_{\epsilon}^{-}(t, c)}^{y_{\epsilon}^{+}(t, c)} \pi_{\epsilon}(t, c, y) \mathrm{d} y \mathrm{~d} t
$$

Furthermore

$$
\{c\} \times\left(0, y_{\epsilon}^{+}(t, c)\right) \subseteq G_{\epsilon}(t) .
$$

Proof of Lemma 7.1. The basic ingredient is the following integral identity: For any test velocity field $\tilde{u}$ we have

$$
\begin{aligned}
\frac{1}{\epsilon^{2}}\left\{\int_{\partial \Omega_{\epsilon}(t) \cap\{y>0\}} \tau \cdot \mathrm{D} \tilde{u} \tau-\int_{\partial \Omega_{\epsilon}(t) \cap\{y=0\}}\left(\begin{array}{l}
1 \\
0
\end{array}\right) \cdot \mathrm{D} \tilde{u}\left(\begin{array}{l}
1 \\
0
\end{array}\right)\right\} \\
=\frac{1}{\epsilon}\left\{\int_{\Omega_{\epsilon}(t)}\left(-u_{\epsilon}(t) \cdot \tilde{u}+p_{\epsilon}(t) \nabla \cdot \tilde{u}\right)-\int_{\partial \Omega_{\epsilon}(t) \cap\{y=0\}} p_{\epsilon}(t)\left(\begin{array}{c}
0 \\
-1
\end{array}\right) \cdot \tilde{u}\right\} .
\end{aligned}
$$

We obtain (7.10) as follows: Because of $\kappa \nu=-\partial_{\tau} \tau$, an integration by parts along $\partial \Omega_{\epsilon}(t) \cap\{y>0\}$ yields

$$
\int_{\partial \Omega_{\epsilon}(t) \cap\{y>0\}} \kappa v \cdot \tilde{u}=\int_{\partial \Omega_{\epsilon}(t) \cap\{y>0\}} \tau \cdot \mathrm{D} \tilde{u} \tau-\int_{\partial\left(\partial \Omega_{\epsilon}(t) \cap\{y>0\}\right)} \tau \cdot \tilde{u} .
$$

On the other hand, an integration by parts along $\partial \Omega_{\epsilon}(t) \cap\{y=0\}$ gives

$$
\int_{\partial \Omega_{\epsilon}(t) \cap\{y=0\}}\left(\begin{array}{l}
1 \\
0
\end{array}\right) \cdot \mathrm{D} \tilde{u}\left(\begin{array}{l}
1 \\
0
\end{array}\right)=\int_{\partial\left(\partial \Omega_{\epsilon}(t) \cap\{y=0\}\right)}\left(\begin{array}{l}
1 \\
0
\end{array}\right) \cdot \tilde{u} .
$$

According to 3.6 we have $\tau=\left(\begin{array}{l}1 \\ 0\end{array}\right)$ on $\partial\left(\partial \Omega_{\epsilon}(t) \cap\{y>0\}\right)=\partial\left(\partial \Omega_{\epsilon}(t) \cap\{y=0\}\right)$, so that

$$
\int_{\partial \Omega_{\epsilon}(t) \cap\{y>0\}} \kappa v \cdot \tilde{u}=\int_{\partial \Omega_{\epsilon}(t) \cap\{y>0\}} \tau \cdot \mathrm{D} \tilde{u} \tau-\int_{\partial \Omega_{\epsilon}(t) \cap\{y=0\}}\left(\begin{array}{l}
1 \\
0
\end{array}\right) \cdot \mathrm{D} \tilde{u}\left(\begin{array}{l}
1 \\
0
\end{array}\right) .
$$

By the divergence theorem, we obtain

$$
\begin{aligned}
\int_{\partial \Omega_{\epsilon}(t) \cap\{y>0\}} p_{\epsilon}(t) v \cdot \tilde{u} & =\int_{\partial \Omega_{\epsilon}(t)} p_{\epsilon}(t) v \cdot \tilde{u}-\int_{\partial \Omega_{\epsilon}(t) \cap\{y=0\}} p_{\epsilon}(t)\left(\begin{array}{c}
0 \\
-1
\end{array}\right) \cdot \tilde{u} \\
& =\int_{\Omega_{\epsilon}(t)}\left(\nabla p_{\epsilon}(t) \cdot \tilde{u}+p_{\epsilon}(t) \nabla \cdot \tilde{u}\right)-\int_{\partial \Omega_{\epsilon}(t) \cap\{y=0\}} p_{\epsilon}(t)\left(\begin{array}{c}
0 \\
-1
\end{array}\right) \cdot \tilde{u}
\end{aligned}
$$

Obviously, (7.10) follows from (7.11) and 7.12 via the identities 3.5 and 3.7 .

We make use of the renormalized version of $(7.10)$ for test fields of the simple form

$$
\tilde{u}_{\epsilon}(t, x, y)=\left(0, \epsilon \tilde{w}_{\epsilon}(t, x)\right),
$$


with $\tilde{w}_{\epsilon}$ satisfying (7.2)-7.4. For the right hand side of (7.10) we obtain

$$
\begin{aligned}
\frac{1}{\epsilon}\left\{\int_{\Omega_{\epsilon}(t)}\left(-u_{\epsilon}(t) \cdot \tilde{u}_{\epsilon}+p_{\epsilon}(t) \nabla \cdot \tilde{u}_{\epsilon}\right)-\int_{\partial \Omega_{\epsilon}(t) \cap\{y=0\}}\right. & \left.p_{\epsilon}(t)\left(\begin{array}{c}
0 \\
-1
\end{array}\right) \cdot \tilde{u}_{\epsilon}\right\} \\
& =\int_{G_{\epsilon}(t)}-\epsilon^{2} w_{\epsilon}(t) \tilde{w}_{\epsilon}(t)+\int_{I \times\{0\}} \pi_{\epsilon}(t) \tilde{w}_{\epsilon}(t) .
\end{aligned}
$$

For the left hand side, we fix $0<\delta \leqslant 1$ and let $\tau=\left(\tau_{1}, \tau_{2}\right)$; in view of (7.4), we may use (4.2) to write

$$
\begin{aligned}
\frac{1}{\epsilon^{2}}\left\{\int_{\partial \Omega_{\epsilon}(t) \cap\{y>0\}} \tau \cdot \mathrm{D} \tilde{u} \tau-\right. & \left.\int_{\partial \Omega_{\epsilon}(t) \cap\{y=0\}}\left(\begin{array}{l}
1 \\
0
\end{array}\right) \cdot \mathrm{D} \tilde{u}\left(\begin{array}{l}
1 \\
0
\end{array}\right)\right\} \\
& =\frac{1}{\epsilon} \int_{\partial \Omega_{\epsilon}(t) \cap\{y>0\}} \tilde{w}_{\epsilon}^{\prime} \tau_{1} \tau_{2}=\int_{U_{\delta, \epsilon}(t) \cap I} \frac{h_{\epsilon}^{\prime}(t)}{\sqrt{1+\left(\epsilon h_{\epsilon}^{\prime}(t)\right)^{2}}} \tilde{w}_{\epsilon}^{\prime}(t) .
\end{aligned}
$$

By convexity of $z \mapsto \frac{1}{\epsilon^{2}}\left(\sqrt{1+(\epsilon z)^{2}}-1\right)$ we see that

$$
\begin{aligned}
\int_{U_{\delta, \epsilon(t)} \cap I} \frac{1}{\epsilon^{2}}\left(\sqrt{1+\left(\epsilon \tilde{h}_{\epsilon}^{\prime}(t)\right)^{2}}-1\right)-\int_{U_{\delta, \epsilon}(t) \cap I} \frac{1}{\epsilon^{2}}\left(\sqrt{1+\left(\epsilon h_{\epsilon}^{\prime}(t)\right)^{2}}-1\right) \\
\quad \geqslant \int_{U_{\delta, \epsilon}(t) \cap I} \frac{h_{\epsilon}^{\prime}(t)}{\sqrt{1+\left(\epsilon h_{\epsilon}^{\prime}(t)\right)^{2}}} \tilde{w}_{\epsilon}^{\prime}(t) .
\end{aligned}
$$

Reassembling the different parts of (7.10) and integrating over $t \in J$ we obtain (7.1).

Proof of Lemma 7.2 Our construction is based on the assumption that the boundary of the set $\Omega_{\epsilon}^{0}(t)=\Omega_{\epsilon}(t) \cup\{y \leqslant 0\}$ is, according to 2.1 , a connected and continuously differentiable curve. We may then introduce a parametrization of this curve by arc length

$$
(-\infty, \infty) \ni s \mapsto \gamma_{\epsilon}(t, s)=\left(x_{\epsilon}(t, s), y_{\epsilon}(t, s)\right),
$$

oriented so that $\lim _{s \rightarrow \pm \infty} x_{\epsilon}(t, s)= \pm \infty$. For notational convenience we neglect dependence on $c$ whenever no ambiguity arises. We would like to construct a set $\Omega_{\epsilon}^{*}(t)$ which is "close" to

$$
\Omega_{\epsilon}^{(c)}(t):=\Omega_{\epsilon}(t) \cap\{c \leqslant x \leqslant b(t)\}
$$

and which has tangent $\left(\begin{array}{l}1 \\ 0\end{array}\right)$ at its "lower right corner". With this in mind, we introduce the arc coordinate $s_{\epsilon}^{(l)}(t)$ (cf. Fig. 4) such that

$$
x_{\epsilon}\left(t, s_{\epsilon}^{(l)}(t)\right)=c \quad \text { and } \quad\{c\} \times\left(0, y_{\epsilon}\left(t, s_{\epsilon}^{(l)}(t)\right)\right) \subset \Omega_{\epsilon}(t),
$$

and we split $J$ into three subintervals: $J_{1}$, where $b(t)=\infty ; J_{2}$, where $h(t) \equiv 0$ in $[b(t), \infty)$; and $J_{3}=J-\left(J_{1} \cup J_{2}\right)$.

We first treat the easiest case $b(t)=\infty$, which already allows outlining the main ideas. In this case we introduce the set

$\Omega_{\epsilon}^{*}(t):=$ portion of $\Omega_{\epsilon}(t)$ bounded by the curves

$$
\gamma\left(t,\left[s_{\epsilon}^{(l)}(t), \infty\right)\right) \text { and }\{c\} \times\left[0, y_{\epsilon}\left(t, s_{\epsilon}^{(l)}(t)\right)\right)
$$




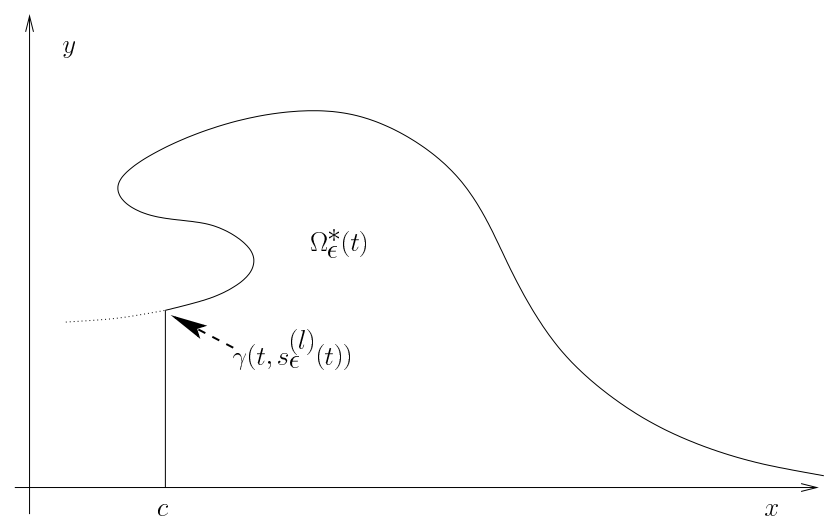

FIG. 4. The arc coordinate $s_{\epsilon}^{(l)}(t)$ and the set $\Omega_{\epsilon}^{*}(t)$ for $t \in J_{1}$.

and its renormalized version

$$
G_{\epsilon}^{*}(t)=\left\{(x, y) \in \mathbb{R} \times(0, \infty):(x, \epsilon y) \in \Omega_{\epsilon}^{*}(t)\right\} .
$$

The set $\Omega_{\epsilon}^{*}(t)$ is constructed in such a way that we can apply the divergence theorem: using the fact that $\kappa \nu=-\partial_{\tau} \tau$ we obtain

$$
\begin{aligned}
\int_{\Omega_{\epsilon}^{*}(t)} u_{\epsilon}(t) \cdot\left(\begin{array}{l}
1 \\
0
\end{array}\right) & =-\int_{\Omega_{\epsilon}^{*}(t)} \nabla \cdot\left(p_{\epsilon}(t), 0\right) \\
& =\int_{0}^{y_{\epsilon}\left(t, s_{\epsilon}^{(l)}(t)\right)} p_{\epsilon}(t, c, y) \mathrm{d} y-\int_{\gamma\left(t,\left[s_{\epsilon}^{(l)}(t), \infty\right)\right)} p_{\epsilon}(t) v(t) \cdot\left(\begin{array}{l}
1 \\
0
\end{array}\right) \\
& \stackrel{3.5}{=} \int_{0}^{y_{\epsilon}\left(t, s_{\epsilon}^{(l)}(t)\right)} p_{\epsilon}(t, c, y) \mathrm{d} y-\frac{1}{\epsilon} \int_{\gamma\left(t,\left[s_{\epsilon}^{(l)}(t), \infty\right)\right)} \kappa(t) v(t) \cdot\left(\begin{array}{l}
1 \\
0
\end{array}\right) \\
& =\int_{0}^{y_{\epsilon}\left(t, s_{\epsilon}^{(l)}(t)\right)} p_{\epsilon}(t, c, y) \mathrm{d} y+\frac{1}{\epsilon} \int_{\gamma\left(t,\left[s_{\epsilon}^{(l)}(t), \infty\right)\right)} \partial_{\tau(t)} \tau(t) \cdot\left(\begin{array}{l}
1 \\
0
\end{array}\right) \\
& \stackrel{3.6]}{\geqslant} \int_{0}^{y_{\epsilon}\left(t, s_{\epsilon}^{(l)}(t)\right)} p_{\epsilon}(t, c, y) \mathrm{d} y,
\end{aligned}
$$

where in the last line we used the fact that $\tau \cdot\left(\begin{array}{l}1 \\ 0\end{array}\right)=1$ at infinity. We let

$$
y_{\epsilon}^{-}(t, c):=0, \quad y_{\epsilon}^{+}(t, c):=\frac{1}{\epsilon} y_{\epsilon}\left(t, s_{\epsilon}^{(l)}(t)\right) .
$$

Rescaling (7.15) and integrating in time, we obtain (7.8) restricted to $t \in J_{1}$ :

$$
\int_{J_{1}} \int_{G_{\epsilon}^{*}(t)} v_{\epsilon}(t) \mathrm{d} t \geqslant \int_{J_{1}} \int_{y_{\epsilon}^{-}(t, c)}^{y_{\epsilon}^{+}(t, c)} \pi_{\epsilon}(t, c, y) \mathrm{d} y \mathrm{~d} t .
$$


Since (7.7) follows from (4.1) and (5.3), and (7.9) holds by construction, it remains to show that $\Omega_{\epsilon}^{*}(t)$ is close to $\Omega_{\epsilon}^{(c)}(t)$ in the sense that

$$
\frac{1}{\epsilon} \mathcal{L}^{2}\left(\Omega_{\epsilon}^{(c)}(t) \triangle \Omega_{\epsilon}^{*}(t)\right) \stackrel{\epsilon \downarrow 0}{\longrightarrow} 0 .
$$

To this end, we write for the symmetric difference

$$
\Omega_{\epsilon}^{(c)}(t) \triangle \Omega_{\epsilon}^{*}(t)=\left(\Omega_{\epsilon}^{(c)}(t)-\Omega_{\epsilon}^{*}(t)\right) \cup\left(\Omega_{\epsilon}^{*}(t)-\Omega_{\epsilon}^{(c)}(t)\right)=: \Omega_{\epsilon}^{\text {in }}(t) \cup \Omega_{\epsilon}^{\text {out }}(t) .
$$

We claim that both $\Omega_{\epsilon}^{\text {in }}(t)$ and $\Omega_{\epsilon}^{\text {out }}(t)$ are small because, roughly speaking, they consist of "appendices", i.e. connected subsets of $\Omega_{\epsilon}(t)$ which are not subgraphs and are therefore doomed to vanish in the limit. Let us consider $\Omega_{\epsilon}^{\text {in }}(t)$ in detail. By definition (7.14,

$$
\partial \Omega_{\epsilon}^{\text {in }}(t) \subset \gamma_{\epsilon}\left(t,\left(-\infty, s_{\epsilon}^{(l)}(t)\right]\right) \cup\{x=c\} .
$$

On the other hand, in view of $4.2, \Omega_{\epsilon}^{0}(t)$ coincides with the subgraph of the curve $\gamma_{\epsilon}\left(t,\left[s_{\epsilon}^{(l)}(t), \infty\right)\right)$ on $[c, \infty) \cap U_{\delta, \epsilon}(t)$ : therefore the projection $\mathrm{pr}_{x}$ of $\Omega_{\epsilon}^{\text {in }}(t)$ onto the $x$-axis is such that for any $\delta \in(0,1]$,

$$
\operatorname{pr}_{x}\left(\Omega_{\epsilon}^{\text {in }}(t)\right) \subset[c, \infty)-U_{\delta, \epsilon}(t) .
$$

In addition, in view of $4.1,, \Omega_{\epsilon}^{\text {in }}(t)$ is close to the subgraph of $h_{\epsilon}(t)$ :

$$
\Omega_{\epsilon}^{\text {in }}(t) \subset\left\{(x, y): x \in[c, \infty)-U_{\delta, \epsilon}(t), 0<y<\epsilon h_{\epsilon}(t, x)+C_{0} \epsilon^{2} E_{\epsilon}(t)\right\} .
$$

In view of [5.3, this implies that

$$
\frac{1}{\epsilon} \mathcal{L}^{2}\left(\Omega_{\epsilon}^{\mathrm{in}}(t)\right) \leqslant C \mathcal{L}^{1}\left(\mathbb{R}-U_{\delta, \epsilon}(t)\right) \stackrel{4.44}{\leqslant} C \frac{\epsilon^{2}}{\delta^{2}} E_{\epsilon}(t) \stackrel{\epsilon \downarrow 0}{\longrightarrow} 0 .
$$

By the same argument we obtain

$$
\frac{1}{\epsilon} \mathcal{L}^{2}\left(\Omega_{\epsilon}^{\text {out }}(t)\right) \leqslant C \mathcal{L}^{1}\left(\mathbb{R}-U_{\delta, \epsilon}(t)\right) \stackrel{\epsilon \downarrow 0}{\longrightarrow} 0
$$

and (7.17) follows. The renormalized version of (7.17) coincides with (7.6) for $t \in J_{1}$.

For $t \in J_{2}$ we choose the same $\Omega_{\epsilon}^{*}(t)$, and the proof proceeds as before. The only difference is that now $\Omega_{\epsilon}^{\text {out }}(t)$ consists of two portions:

$$
\Omega_{\epsilon}^{\text {out }}(t)=\left(\Omega_{\epsilon}^{\text {out }}(t) \cap\{x \leqslant c\}\right) \cup\left(\Omega_{\epsilon}^{\text {out }}(t) \cap\{x \geqslant b\}\right)=: \Omega_{1, \epsilon}^{\text {out }} \cup \Omega_{2, \epsilon}^{\text {out }} .
$$

The first set consists of "appendices", and its estimating proceeds as before. The second one is small because $h_{\epsilon}(t)$ is uniformly small:

$$
\frac{1}{\epsilon} \mathcal{L}^{2}\left(\Omega_{2, \epsilon}^{\text {out }}(t)\right) \leqslant \frac{1}{\epsilon} \int_{\Omega_{\epsilon}(t) \cap\{x \geqslant b\}} \mathrm{d} x \mathrm{~d} y \leqslant \int_{b}^{\infty}\left(h_{\epsilon}(t, x)+C_{0} \epsilon E_{\epsilon}(t)\right) \mathrm{d} x \stackrel{\epsilon \downarrow 0}{\longrightarrow} 0 .
$$

For $t \in J_{3}$, we have

$$
b_{1}(t):=\sup \left\{x \geqslant b(t): h_{\epsilon}(t, \xi)=0 \forall \xi \in[b, x]\right\}<\infty .
$$




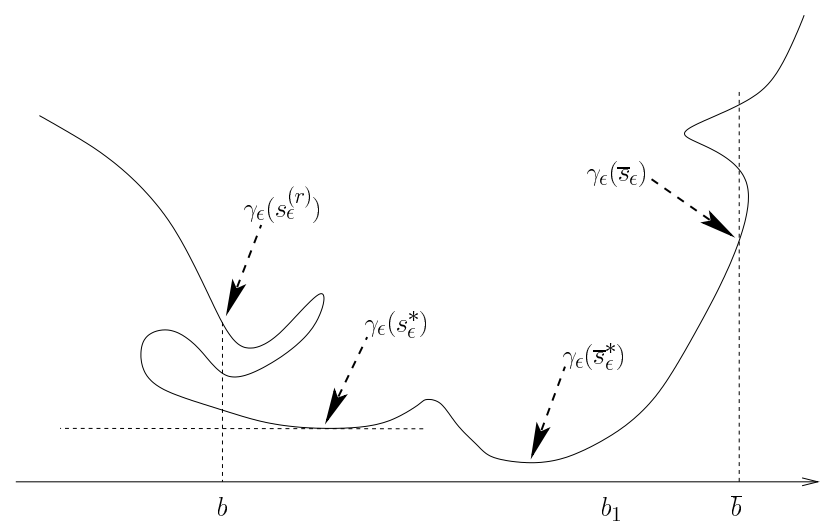

FIG. 5. The arc coordinates $s_{\epsilon}^{(r)}, s_{\epsilon}^{*}, \bar{s}_{\epsilon}$ and $\bar{s}_{\epsilon}^{*}$ for $t \in J_{3}$.

In this case both the choice of $\Omega_{\epsilon}^{*}(t)$ and its estimation are more subtle. For notational convenience we neglect time dependence. Let

$$
s_{\epsilon}^{(r)}=\inf \left\{s: x_{\epsilon}(s)=b\right\}
$$

(cf. Fig. 55. We observe for later reference that

$$
\frac{1}{\epsilon} y_{\epsilon}\left(s_{\epsilon}^{(r)}\right) \stackrel{\text { 4.1. }}{\leqslant} h_{\epsilon}(b)+C_{0} \epsilon E_{\epsilon}(t) \stackrel{\epsilon \downarrow 0}{\rightarrow} 0 .
$$

Let now

$$
\begin{array}{r}
s_{\epsilon}^{*}=\inf \left\{\tilde{s} \geqslant s_{\epsilon}^{(l)}: y_{\epsilon}(\tilde{s}) \leqslant y_{\epsilon}(s) \forall s \in\left[s_{\epsilon}^{(l)}, \max \left\{s_{\epsilon}^{(r)}, \tilde{s}\right\}\right],\right. \\
\left.\frac{\mathrm{d} x_{\epsilon}}{\mathrm{d} s}(\tilde{s})=1,\left[c, x_{\epsilon}(\tilde{s})\right] \times\left\{y_{\epsilon}(\tilde{s})\right\} \subset \bar{\Omega}_{\epsilon}\right\}
\end{array}
$$

(cf. Fig. 57. We will show that the set in (7.20) is not empty for $\epsilon$ sufficiently small, and that moreover

$$
\limsup _{\epsilon \downarrow 0} x_{\epsilon}\left(s_{\epsilon}^{*}\right) \leqslant b_{1} .
$$

To see this, take any $\bar{b}>b_{1}$ such that $h(\bar{b})>0$, and let

$$
\bar{s}_{\epsilon}=\inf \left\{s: x_{\epsilon}(s)=\bar{b}\right\} .
$$

Since $\Omega_{\epsilon}$ coincides with the subgraph of $h_{\epsilon}$ over a set with small complement (in the sense of 4.2) and (4.4) ) and $h_{\epsilon}$ is close to $h$ (in the sense of (5.3p), for $\epsilon$ sufficiently small we must have

$$
y_{\epsilon}\left(\bar{s}_{\epsilon}\right)>y_{\epsilon}\left(s_{\epsilon}^{(r)}\right), \quad y_{\epsilon}\left(s_{\epsilon}^{(l)}\right)>y_{\epsilon}\left(s_{\epsilon}^{(r)}\right), \quad s_{\epsilon}^{(l)}<s_{\epsilon}^{(r)}<\bar{s}_{\epsilon} .
$$

Hence $y_{\epsilon}\left(\left[s_{\epsilon}^{(l)}, \bar{s}_{\epsilon}\right]\right)$ attains its absolute minimum at a point $\bar{s}_{\epsilon}^{*}$ in the interior:

$$
\bar{s}_{\epsilon}^{*} \in\left(s_{\epsilon}^{(l)}, \bar{s}_{\epsilon}\right) .
$$


By (7.19) and (7.23),

$$
\frac{1}{\epsilon} y_{\epsilon}\left(\bar{s}_{\epsilon}^{*}\right) \leqslant \frac{1}{\epsilon} y_{\epsilon}\left(s_{\epsilon}^{(r)}\right) \stackrel{\epsilon \downarrow 0}{\longrightarrow} 0,
$$

which together with 7.22 implies that

$$
\limsup _{\epsilon \downarrow 0} x_{\epsilon}\left(\bar{s}_{\epsilon}^{*}\right)<\bar{b}
$$

Again by (4.2)-(4.4) and (5.3), the limit in (7.25) implies that

$$
\liminf _{\epsilon \downarrow 0} x_{\epsilon}\left(\bar{s}_{\epsilon}^{*}\right)>c .
$$

Of course $\frac{\mathrm{d} y_{\epsilon}}{\mathrm{d} s}\left(\bar{s}_{\epsilon}^{*}\right)=0$. A simple topological argument rules out that $\frac{\mathrm{d} x_{\epsilon}}{\mathrm{d} s}\left(\bar{s}_{\epsilon}^{*}\right)=-1$ : Indeed, in view of 7.13 and $(7.20)$, the curve $\gamma_{\epsilon}\left(\left(\bar{s}_{\epsilon}^{*}, \infty\right)\right)$ would then be "trapped" strictly to the left of $\bar{b}$, contrary to $x_{\epsilon}\left(\bar{s}_{\epsilon}\right)=\bar{b}$. Therefore

$$
\frac{\mathrm{d} x_{\epsilon}}{\mathrm{d} s}\left(\bar{s}_{\epsilon}^{*}\right)=1
$$

Another simple topological argument shows that

$$
A_{\epsilon}:=\left[c, x_{\epsilon}\left(\bar{s}_{\epsilon}^{*}\right)\right] \times\left\{y_{\epsilon}\left(\bar{s}_{\epsilon}^{*}\right)\right\} \subset \bar{\Omega}_{\epsilon}
$$

( $A_{\epsilon}$ is not empty because of 7.27$)$ ). If this were not true, then since $\bar{s}_{\epsilon}^{*}$ minimizes $y_{\epsilon}\left(\left[s_{\epsilon}^{(l)}, \bar{s}_{\epsilon}\right]\right)$ and $\gamma_{\epsilon}\left(\left(-\infty, s_{\epsilon}^{(l)}\right)\right) \cap\left(\{c\} \times\left(0, y_{\epsilon}\left(s_{\epsilon}^{(l)}\right)\right)\right)=\emptyset$, the only way for $\gamma_{\epsilon}$ to reach $A_{\epsilon}$ would be to go from $\bar{b}$ to the left of $x_{\epsilon}\left(\bar{s}_{\epsilon}^{*}\right)$. Therefore $\Omega_{\epsilon}$ would not be a subgraph over the entire interval $x \in\left(x_{\epsilon}\left(\bar{s}_{\epsilon}^{*}\right), \bar{b}\right)$, which in view of (7.26) has uniformly positive measure with respect to $\epsilon$. This contradicts $(4.2)$ and proves (7.29). Properties $(7.23),(7.28)$ and $(7.29)$ imply that $\bar{s}_{\epsilon}^{*}$ belongs to the set in $(7.20)$, which therefore is not empty. Since $b \in\left(b_{1}, \infty\right) \cap\{h>0\}$ is arbitrary, 7.26) and definition 7.18 imply 7.21. In addition, 7.19) yields

$$
\frac{1}{\epsilon} y_{\epsilon}\left(s_{\epsilon}^{*}\right) \leqslant \frac{1}{\epsilon} y_{\epsilon}\left(s_{\epsilon}^{(r)}\right) \stackrel{\epsilon \downarrow 0}{\longrightarrow} 0 .
$$

Definition 7.20 of $s_{\epsilon}^{*}$ allows us to introduce the desired set $\Omega_{\epsilon}^{*}$ :

$$
\begin{aligned}
& \Omega_{\epsilon}^{*}=\text { portion of } \Omega_{\epsilon} \text { bounded by the curves } \\
& \qquad \gamma\left(\left[s_{\epsilon}^{(l)}, s_{\epsilon}^{*}\right]\right),\{c\} \times\left[y_{\epsilon}\left(s_{\epsilon}^{*}\right), y_{\epsilon}\left(s_{\epsilon}^{(l)}\right)\right) \text { and }\left[c, x_{\epsilon}\left(s_{\epsilon}^{*}\right)\right] \times\left\{y_{\epsilon}\left(s_{\epsilon}^{*}\right)\right\} .
\end{aligned}
$$

Since $v=\left(\begin{array}{c}0 \\ -1\end{array}\right)$ on the additional segment of the boundary curve, the proof of the integral inequality is identical to the case $t \in J_{1}$ with

$$
y_{\epsilon}^{-}(t, c)=\frac{1}{\epsilon} y_{\epsilon}\left(t, s_{\epsilon}^{*}(t, c)\right), \quad y_{\epsilon}^{+}(t, c)=\frac{1}{\epsilon} y_{\epsilon}\left(t, s_{\epsilon}^{(l)}(t, c)\right),
$$




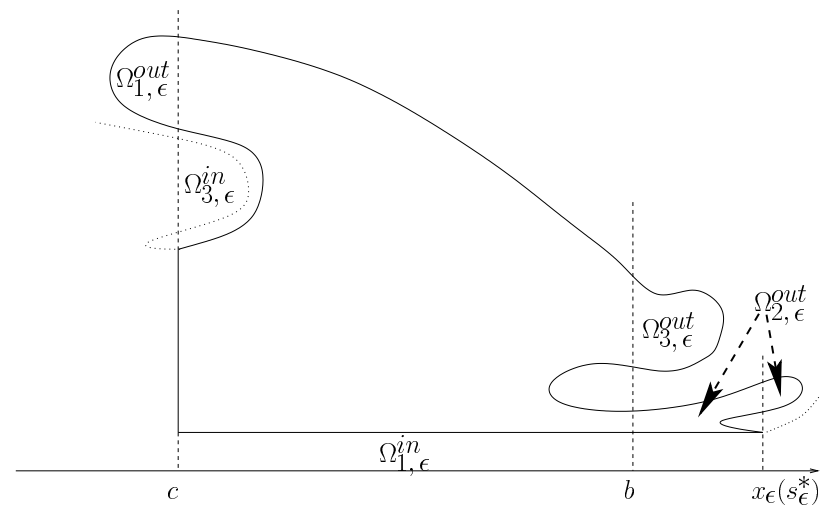

FIG. 6. The splitting of the symmetric difference $\Omega_{\epsilon}^{(c)} \triangle \Omega_{\epsilon}^{*}$ for $t \in J_{3}$. Here $x_{\epsilon}\left(s_{\epsilon}^{*}\right)>b$, thus $\Omega_{2, \epsilon}^{\text {in }}$ is empty.

and (7.7), (7.9) follow as before if we also take (7.30) into account. Our last task is to show that $\Omega_{\epsilon}^{*}(t)$ is close to $\Omega_{\epsilon}^{(c)}(t)$. To this end, we split the symmetric difference as follows (cf. Fig. 6):

$$
\begin{aligned}
\Omega_{\epsilon}^{\text {in }}= & \left(\Omega_{\epsilon}^{\text {in }} \cap\left(\left[c, \min \left\{x_{\epsilon}\left(s_{\epsilon}^{*}\right), b\right\}\right] \times\left[0, y_{\epsilon}\left(s_{\epsilon}^{*}\right)\right)\right)\right) \\
& \cup\left(\Omega_{\epsilon}^{\text {in }} \cap\left\{x_{\epsilon}\left(s_{\epsilon}^{*}\right)<x \leqslant b\right\}\right) \\
& \cup\left(\Omega_{\epsilon}^{\text {in }} \cap\left(\left[c, \min \left\{x_{\epsilon}\left(s_{\epsilon}^{*}\right), b\right\}\right] \times\left[y_{\epsilon}\left(s_{\epsilon}^{*}\right), \infty\right)\right)\right) \\
= & : \Omega_{1, \epsilon}^{\text {in }} \cup \Omega_{2, \epsilon}^{\text {in }} \cup \Omega_{3, \epsilon}^{\text {in }},
\end{aligned}
$$

and

$$
\Omega_{\epsilon}^{\text {out }}=\Omega_{1, \epsilon}^{\text {out }} \cup \Omega_{2 \epsilon}^{\text {out }} \cup \Omega_{3, \epsilon}^{\text {out }},
$$

where $\Omega_{1, \epsilon}^{\text {out }}=\Omega_{\epsilon}^{*} \cap\{x \leqslant c\}, \Omega_{2, \epsilon}^{\text {out }}$ is the (possibly empty) connected component of $\Omega_{\epsilon}^{*} \cap\{x \geqslant b\}$ whose boundary contains $\left(x_{\epsilon}\left(s_{\epsilon}^{*}\right), y_{\epsilon}\left(s_{\epsilon}^{*}\right)\right)$, and $\Omega_{3, \epsilon}^{\text {out }}$ is the remaining part of $\Omega_{\epsilon}^{*} \cap\{x \geqslant b\}$. We are going to argue that each of these sets is small in the sense that

$$
\limsup _{\epsilon \downarrow 0} \frac{1}{\epsilon} \mathcal{L}^{2}\left(\Omega_{j, \epsilon}^{\text {in }}\right)=\limsup _{\epsilon \downarrow 0} \frac{1}{\epsilon} \mathcal{L}^{2}\left(\Omega_{j, \epsilon}^{\text {out }}\right)=0, \quad j=1,2,3 .
$$

It is easy to check that $\Omega_{3, \epsilon}^{\text {in }}, \Omega_{1, \epsilon}^{\text {out }}$ and $\Omega_{3, \epsilon}^{\text {out }}$ consist of "appendices", hence for these sets the proof of 7.31 proceeds as before. The set $\Omega_{1, \epsilon}^{\text {in }}$ is small in view of 7.30):

$$
\frac{1}{\epsilon} \mathcal{L}^{2}\left(\Omega_{1, \epsilon}^{\text {in }}\right) \leqslant(b-c) \frac{1}{\epsilon} y_{\epsilon}\left(s_{\epsilon}^{*}\right) \stackrel{\epsilon \downarrow 0}{\longrightarrow} 0 .
$$

For $\Omega_{2, \epsilon}^{\text {in }}$, we use the fact that $x_{\epsilon}\left(s_{\epsilon}^{*}\right)$ is at least as large as $b$ in the limit:

$$
\liminf _{\epsilon \downarrow 0} x_{\epsilon}\left(s_{\epsilon}^{*}\right) \geqslant b .
$$

This follows easily from $(7.21)$ and 7.30 — which imply that the limit of any subsequence must lie in $(-\infty, c] \cup\left[b, b_{1}\right)$-and from the fact that $\Omega_{\epsilon}$ is almost a subgraph. Therefore also $\Omega_{2, \epsilon}^{\text {in }}$ consists 


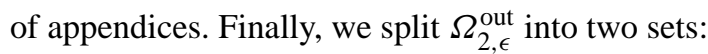

$$
\Omega_{2, \epsilon}^{\text {out }}=\left(\Omega_{2, \epsilon}^{\text {out }} \cap\left\{x \leqslant x_{\epsilon}\left(s_{\epsilon}^{*}\right)\right\}\right) \cup\left(\Omega_{2, \epsilon}^{\text {out }} \cap\left\{x>x_{\epsilon}\left(s_{\epsilon}^{*}\right)\right\}\right) .
$$

The second set consists of appendices. The first one is small since $h \equiv 0$ in $\left[b, b_{1}\right]$ : Indeed, for each $\delta>0$ and $\epsilon$ sufficiently small

$$
\left.\Omega_{2, \epsilon}^{\text {out }} \cap\left\{x \leqslant x_{\epsilon}\left(s_{\epsilon}^{*}\right)\right\} \subset\left\{(x, y): b \leqslant x \leqslant b_{1}+\delta, 0<y<\epsilon h_{\epsilon}(x)+C_{0} \epsilon^{2} E_{\epsilon}(t)\right)\right\}
$$

so that

$$
\frac{1}{\epsilon} \mathcal{L}^{2}\left(\Omega_{2, \epsilon}^{\text {out }} \cap\left\{x \leqslant x_{\epsilon}\left(s_{\epsilon}^{*}\right)\right\}\right) \leqslant\left(b_{1}-b+\delta\right)\left(\sup _{x \in\left(b, b_{1}+\delta\right)} h_{\epsilon}(x)+C_{0} \epsilon E_{\epsilon}(0)\right),
$$

and taking the limit $\epsilon \downarrow 0$ and $\delta \downarrow 0$ (in this order) yields

$$
\frac{1}{\epsilon} \mathcal{L}^{2}\left(\Omega_{2, \epsilon}^{\text {out }} \cap\left\{x \leqslant x_{\epsilon}\left(s_{\epsilon}^{*}\right)\right\}\right) \stackrel{\epsilon \downarrow 0}{\longrightarrow} 0 .
$$

Therefore (7.31) holds and the proof is complete.

\section{Pressure neutrality and passage to the limit}

In this section, we pass to the limit in the variational formulations of Lemmas 7.1 and 7.2 Both formulations involve the pressure $\pi_{\epsilon}$ within one connected component of $\{h>0\}$. Unfortunately, in the limit $\epsilon \downarrow 0$, we only control $\pi_{\epsilon}$ up to an additive constant within the connected component of $\{h>0\}$ (see Section 6). Hence we have to combine both test fields in such a way that the outcome is oblivious to the absolute pressure, i.e. that it just depends on the well-controlled relative pressure $\hat{\pi}_{\epsilon}$ introduced in 6.6. We call this "pressure neutrality". In view of the pressure's role as a Lagrange multiplier enforcing the incompressibility constraint, it is not surprising that "pressure neutrality" amounts to the requirement (8.4) that the combined variation preserves the total area.

PROPOSITION 8.1 For almost every $t>0$, let $I \subseteq \mathbb{R}$ be such that $h(t)>0$ in its closure, $v_{0} \geqslant 0$, $c \in I$, and $b(t, c)$ defined by $(7.5)$. Then

$$
\int_{I}\left\{\frac{1}{2}\left(\tilde{h}^{\prime}(t)\right)^{2}-\frac{1}{2}\left(h^{\prime}(t)\right)^{2}\right\}+v_{0} \int_{c}^{b(t, c)} h(t) v(t) \geqslant \int_{I} \hat{\pi}(t) \tilde{w}+v_{0} h(t, c) \hat{\pi}(t, c)
$$

for all $\tilde{w} \in H^{1}(\mathbb{R})$ with

$$
\begin{aligned}
\tilde{h}(t) & :=h(t)+\tilde{w}, \\
\tilde{w} & =0 \quad \text { outside of } I, \\
\int_{I} \tilde{w} & =-v_{0} h(t, c) .
\end{aligned}
$$

Note that all terms in (8.1) are well defined for almost every $t>0$ in view of $(5.5),(6.9)$ and Remark 6.3. In view of the continuity in time of $h(t, c)$ on the right hand side of 8.4), Proposition 8.1 follows via a density argument from the following time integrated version: 
LEMmA 8.2 Let $J \times I \subset(0, \infty) \times \mathbb{R}$ be an open rectangle such that $\{h>0\}$ in its closure, $v_{0} \geqslant 0$, $c \in I$, and $b(t, c)$ defined by $(7.5)$. Then

$$
\int_{J \times I}\left\{\frac{1}{2}\left(\tilde{h}^{\prime}\right)^{2}-\frac{1}{2}\left(h^{\prime}\right)^{2}\right\}+v_{0} \int_{J} \int_{c}^{b(t, c)} h(t) v(t) \mathrm{d} t \geqslant \int_{J \times I} \hat{\pi} \tilde{w}+v_{0} \int_{J} h(c) \hat{\pi}(c)
$$

for all $\tilde{w} \in L^{2}\left(J ; H^{1}(\mathbb{R})\right)$ with

$$
\begin{aligned}
\tilde{h} & :=h+\tilde{w}, \\
\tilde{w}(t) & =0 \quad \text { outside of } I \text { for a.e. } t \in J, \\
\int_{I} \tilde{w}(t) & =-v_{0} h(t, c) \quad \text { for a.e. } t \in J .
\end{aligned}
$$

Proof. The basic ingredients are 7.8 ,

$$
\int_{J} \int_{G_{\epsilon}^{*}(t, c)} v_{\epsilon}(t) \mathrm{d} t \geqslant \int_{J} \int_{y_{\epsilon}^{-}(t, c)}^{y_{\epsilon}^{+}(t, c)} \pi_{\epsilon}(t, c, y) \mathrm{d} y \mathrm{~d} t,
$$

and 7.1 ,

$$
\begin{aligned}
\int_{U_{\delta, \epsilon} \cap(J \times I)} \frac{1}{\epsilon^{2}}\left(\sqrt{1+\left(\epsilon \tilde{h}_{\epsilon}^{\prime}\right)^{2}}-1\right)-\int_{U_{\delta, \epsilon} \cap(J \times I)} \frac{1}{\epsilon^{2}}\left(\sqrt{1+\left(\epsilon h_{\epsilon}^{\prime}\right)^{2}}-1\right) & \\
& \geqslant \int_{G_{\epsilon} \cap(J \times I)}-\epsilon^{2} w_{\epsilon} \tilde{w}_{\epsilon}+\int_{J \times I \times\{0\}} \pi_{\epsilon} \tilde{w}_{\epsilon},
\end{aligned}
$$

which holds for test functions satisfying

$$
\begin{aligned}
\tilde{h}_{\epsilon} & =h_{\epsilon}+\tilde{w}_{\epsilon}, \\
\tilde{w}_{\epsilon}(t) & =0 \quad \text { outside of } I \text { for a.e. } t \in J, \\
\tilde{w}_{\epsilon}^{\prime}(t) & =0 \quad \text { outside of } U_{\delta, \epsilon}(t) \text { for a.e. } t \in J .
\end{aligned}
$$

If we multiply 8.9 by a non-negative constant $v_{0}$ and sum it with 8.10 , we get

$$
\begin{gathered}
\int_{U_{\delta, \epsilon} \cap(J \times I)} \frac{1}{\epsilon^{2}}\left(\sqrt{1+\left(\epsilon \tilde{h}_{\epsilon}^{\prime}\right)^{2}}-1\right)-\int_{U_{\delta, \epsilon} \cap(J \times I)} \frac{1}{\epsilon^{2}}\left(\sqrt{1+\left(\epsilon h_{\epsilon}^{\prime}\right)^{2}}-1\right)+v_{0} \int_{J} \int_{G_{\epsilon}^{*}(t, c)} v_{\epsilon}(t) \mathrm{d} t \\
\geqslant \int_{G_{\epsilon} \cap(J \times I)}-\epsilon^{2} w_{\epsilon} \tilde{w}_{\epsilon}+\int_{J \times I \times\{0\}} \pi_{\epsilon} \tilde{w}_{\epsilon}+v_{0} \int_{J} \int_{y_{\epsilon}^{-}(t, c)}^{y_{\epsilon}^{+}(t, c)} \pi_{\epsilon}(t, c, y) \mathrm{d} y \mathrm{~d} t .
\end{gathered}
$$

We now introduce the $\epsilon$-counterpart of the "neutrality" condition 8.8 for $\tilde{w}_{\epsilon}$ :

$$
\int_{I} \tilde{w}_{\epsilon}(t)=-v_{0}\left(y_{\epsilon}^{+}(t, c)-y_{\epsilon}^{-}(t, c)\right)=:-\lambda_{\epsilon}(t) \quad \text { for a.e. } t \in J .
$$

In view of 7.9 and the definitions $6.5,6.6$ of $\underline{G}_{\epsilon}, \hat{\pi}_{\epsilon}$, we have

$$
\pi_{\epsilon}(t, c, y)=\hat{\pi}_{\epsilon}(t, c, y)+f_{I \times\left(0, h_{0}\right)} \pi_{\epsilon}(t) \quad \text { for a.e. } t \in J, y \in\left(y_{\epsilon}^{-}(t, c), y_{\epsilon}^{+}(t, c)\right),
$$


whence

$$
\begin{aligned}
& v_{0} \int_{J} \int_{y_{\epsilon}^{-}(t, c)}^{y_{\epsilon}^{+}(t, c)} \pi_{\epsilon}(t, c, y) \mathrm{d} y \mathrm{~d} t+\int_{J \times I \times\{0\}} \pi_{\epsilon} \tilde{w}_{\epsilon} \\
& \quad=v_{0} \int_{J} \int_{y_{\epsilon}^{-}(t, c)}^{y_{\epsilon}^{+}(t, c)} \hat{\pi}_{\epsilon}(t, c, y) \mathrm{d} y \mathrm{~d} t+v_{0} \int_{J} \int_{y_{\epsilon}^{-}(t, c)}^{y_{\epsilon}^{+}(t, c)} f_{I \times\left(0, h_{0}\right)} \pi_{\epsilon}+\int_{J \times I \times\{0\}} \pi_{\epsilon} \tilde{w}_{\epsilon} \\
& \stackrel{8.15}{=} v_{0} \int_{J} \int_{y_{\epsilon}^{-}(t, c)}^{y_{\epsilon}^{+}(t, c)} \hat{\pi}_{\epsilon}(t, c, y) \mathrm{d} y \mathrm{~d} t+\int_{J \times I \times\{0\}} \hat{\pi}_{\epsilon} \tilde{w}_{\epsilon} .
\end{aligned}
$$

Substituting 8.16 into 8.14 we conclude that the inequality

$$
\begin{gathered}
\int_{U_{\delta, \epsilon} \cap(J \times I)} \frac{1}{\epsilon^{2}}\left(\sqrt{1+\left(\epsilon \tilde{h}_{\epsilon}^{\prime}\right)^{2}}-1\right)-\int_{U_{\delta, \epsilon} \cap(J \times I)} \frac{1}{\epsilon^{2}}\left(\sqrt{1+\left(\epsilon h_{\epsilon}^{\prime}\right)^{2}}-1\right)+v_{0} \int_{J} \int_{G_{\epsilon}^{*}(t, c)} v_{\epsilon}(t) \mathrm{d} t \\
\geqslant \int_{G_{\epsilon} \cap(J \times I)}-\epsilon^{2} w_{\epsilon} \tilde{w}_{\epsilon}+\int_{J \times I \times\{0\}} \hat{\pi}_{\epsilon} \tilde{w}_{\epsilon}+v_{0} \int_{J} \int_{y_{\epsilon}^{-}(t, c)}^{y_{\epsilon}^{+}(t, c)} \hat{\pi}_{\epsilon}(t, c, y) \mathrm{d} y \mathrm{~d} t
\end{gathered}
$$

holds for any $v_{0} \geqslant 0$ and any function $\tilde{w}_{\epsilon} \in L^{2}\left(J ; H^{1}(\mathbb{R})\right)$ such that $8.11,8.12$, 8.13 and (8.15) are satisfied.

From this, we hope to recover 8.5 in the limit $\epsilon \downarrow 0, \delta \downarrow 0$ (in this order). More precisely, we think of $\tilde{h}_{\epsilon}$ and $\tilde{w}_{\epsilon}$ as approximations to $\tilde{h}$ and $\tilde{w} \in L^{2}\left(J ; H^{1}(\mathbb{R})\right)$, and will now infer which type of convergence is required. Then we will construct these approximations in such a way that (8.11), 8.12, 8.13 and 8.15) are satisfied. Let us first treat the terms on the left hand side of 8.17). We recall the result stated in (5.3), (5.6), 6.7) and (7.6):

$$
\begin{gathered}
h_{\epsilon} \stackrel{\epsilon \downarrow 0}{\rightarrow} h \quad \text { uniformly in }[0, T] \times \mathbb{R} \text { for all } T<\infty, \\
\left\{\begin{array}{ll}
h_{\epsilon}^{\prime} & \text { in } U_{\delta, \epsilon} \cap(J \times I) \\
0 & \text { elsewhere }
\end{array}\right\} \stackrel{\epsilon \downarrow 0}{\rightarrow} h^{\prime} \quad \text { in } L^{2}(J \times I), \\
v_{\epsilon} \stackrel{\epsilon \downarrow 0}{\rightarrow} v \quad \text { in } L^{2}((0, \infty) \times \mathbb{R} \times(0, \infty)), \\
\lim _{\epsilon \downarrow 0} \mathcal{L}^{2}\left(G_{\epsilon}^{*}(t, c) \triangle G_{\epsilon}^{(c)}(t)\right)=0 \quad \forall t \in J .
\end{gathered}
$$

For the last term on the left hand side of 8.17 we have, using Hölder's inequality,

$$
\left|\int_{J} \int_{G_{\epsilon}^{*}(t, c)} v_{\epsilon}(t) \mathrm{d} t-\int_{J} \int_{G_{\epsilon}^{(c)}(t)} v_{\epsilon}(t) \mathrm{d} t\right| \leqslant\left(\int_{J} \mathcal{L}^{2}\left(G_{\epsilon}^{*}(t, c) \Delta G_{\epsilon}^{(c)}(t)\right) \mathrm{d} t\right)^{1 / 2}\left(\int_{G_{\epsilon}}\left|v_{\epsilon}\right|^{2}\right)^{1 / 2} .
$$

According to $8.20,8.21$ and by dominated convergence (in $t$ ), the right hand side converges to zero for $\epsilon \downarrow 0$, and on the other hand by 8.18$)$, (8.20),

$$
\int_{J} \int_{G_{\epsilon}^{(c)}(t)} v_{\epsilon}(t) \mathrm{d} t \stackrel{\epsilon \downarrow 0}{\longrightarrow} \int_{J} \int_{c}^{b(t, c)} h(t) v(t) \mathrm{d} t .
$$

Therefore

$$
\int_{J} \int_{G_{\epsilon}^{*}(t, c)} v_{\epsilon}(t) \mathrm{d} t \stackrel{\epsilon \downarrow 0}{\longrightarrow} \int_{J} \int_{c}^{b(t, c)} h(t) v(t) \mathrm{d} t .
$$


For the middle term on the left hand side of 8.17 we observe that

$$
\frac{1}{\epsilon^{2}}\left(\sqrt{1+(\epsilon z)^{2}}-1\right) \geqslant c_{\delta} \frac{1}{2} z^{2} \text { for }|\epsilon z| \leqslant \delta
$$

where

$$
c_{\delta}=\frac{2}{\delta^{2}}\left(\sqrt{1+\delta^{2}}-1\right) \stackrel{\delta \downarrow 0}{\longrightarrow} 1,
$$

so that, recalling the definition 4.19 of $U_{\delta, \epsilon}$,

$$
\int_{U_{\delta, \epsilon} \cap(J \times I)} \frac{1}{\epsilon^{2}}\left(\sqrt{1+\left(\epsilon h_{\epsilon}^{\prime}\right)^{2}}-1\right) \geqslant c_{\delta} \int_{U_{\delta, \epsilon} \cap(J \times I)} \frac{1}{2}\left(h_{\epsilon}^{\prime}\right)^{2} .
$$

Since, as is well-known, 8.19 implies

$$
\int_{J \times I} \frac{1}{2}\left(h^{\prime}\right)^{2} \leqslant \liminf _{\epsilon \downarrow 0} \int_{U_{\delta, \epsilon} \cap(J \times I)} \frac{1}{2}\left(h_{\epsilon}^{\prime}\right)^{2},
$$

we obtain for the middle term on the left hand side of 8.17

$$
\int_{J \times I} \frac{1}{2}\left(h^{\prime}\right)^{2} \leqslant \liminf _{\delta \downarrow 0} \liminf _{\epsilon \downarrow 0} \int_{U_{\delta, \epsilon} \cap(J \times I)} \frac{1}{\epsilon^{2}}\left(\sqrt{1+\left(\epsilon h_{\epsilon}^{\prime}\right)^{2}}-1\right) .
$$

For the first term on the left hand side of 8.17 we observe that

$$
\frac{1}{\epsilon^{2}}\left(\sqrt{1+(\epsilon z)^{2}}-1\right) \stackrel{\epsilon \downarrow 0}{\longrightarrow} \frac{1}{2} z^{2}, \quad 0 \leqslant \frac{1}{\epsilon^{2}}\left(\sqrt{1+(\epsilon z)^{2}}-1\right) \leqslant \frac{1}{2} z^{2} .
$$

Assume that $\tilde{h}_{\epsilon}$ is an approximation to $\tilde{h}$ such that

$$
\left\{\begin{array}{ll}
\tilde{h}_{\epsilon}^{\prime} & \text { in } U_{\delta, \epsilon} \cap(J \times I) \\
0 & \text { elsewhere }
\end{array}\right\} \stackrel{\epsilon \downarrow 0}{\longrightarrow} \tilde{h}^{\prime} \quad \text { in } L^{2}(J \times I) .
$$

Then, by dominated convergence, for the first term on the left hand side of 8.17) we obtain

$$
\int_{J \times I} \frac{1}{2}\left(\tilde{h}^{\prime}\right)^{2}=\lim _{\epsilon \downarrow 0} \int_{U_{\delta, \epsilon} \cap(J \times I)} \frac{1}{\epsilon^{2}}\left(\sqrt{1+\left(\epsilon \tilde{h}_{\epsilon}^{\prime}\right)^{2}}-1\right) .
$$

Now consider the terms on the right hand side of 8.17). We recall, as stated in Proposition 6.1 (iii), (iv), and in (7.7), that

$$
\begin{gathered}
\hat{\pi}_{\epsilon}(\cdot, \cdot, 0) \stackrel{\epsilon \downarrow 0}{\longrightarrow} \hat{\pi} \quad \text { in } L^{2}(J \times I), \\
\hat{\pi}_{\epsilon}(\cdot, c, \cdot) \rightarrow \hat{\pi}(t, c) \chi(0, h(t, c))(y) \quad \text { in } L^{p}(J \times(0, \infty)) \text { for a.e. } c \in I, \\
\lim _{\epsilon \downarrow 0}\left(y_{\epsilon}^{-}(t, c), y_{\epsilon}^{+}(t, c)\right)=(0, h(t, c)) \quad \text { uniformly for } t \in J,
\end{gathered}
$$

with $p \in[1,2)$. Let us assume that

$$
\tilde{w}_{\epsilon} \stackrel{\epsilon \downarrow 0}{\longrightarrow} \tilde{w} \quad \text { in } L^{2}((0, \infty) \times \mathbb{R}) .
$$


Since $\tilde{w}_{\epsilon}(t)=0$ outside of $I$ for a.e. $t \in J,(8.23)$ and $(8.26)$ yield

$$
\int_{J \times I \times\{0\}} \hat{\pi} \tilde{w}=\lim _{\epsilon \downarrow 0} \int_{J \times I \times\{0\}} \hat{\pi}_{\epsilon} \tilde{w}_{\epsilon} .
$$

As a consequence of 5.2 ,

$$
\epsilon w_{\epsilon} \quad \text { is bounded in } L^{2}((0, \infty) \times \mathbb{R} \times(0, \infty)) .
$$

Hence 8.26 is also sufficient to ensure that the first term on the right hand side of (8.17) vanishes in the limit:

$$
\lim _{\epsilon \downarrow 0} \epsilon \int_{G_{\epsilon} \cap\{t \in J\}}\left(\epsilon w_{\epsilon}\right) \tilde{w}_{\epsilon}=0 .
$$

For the last term on the right hand side of (8.17), we just need to apply 8.24 and 8.25 :

$$
\lim _{\epsilon \downarrow 0} \int_{J} \int_{y_{\epsilon}^{-}(t, c)}^{y_{\epsilon}^{+}(t, c)} \hat{\pi}_{\epsilon}(t, c, y) \mathrm{d} y \mathrm{~d} t=\int_{J} h(t, c) \hat{\pi}(t, c) \mathrm{d} t \quad \text { for a.e. } c \in I .
$$

Combining the limits above, we see that the desired inequality $(8.5)$ holds for almost every $c \in I$ and for any $\tilde{h}$ and $\tilde{w}$ satisfying $(8.6)-8.8$, provided there exist $\hat{h}_{\epsilon}$ and $\tilde{w}_{\epsilon}$ satisfying $8.11-8.13$ and 8.15 for almost every $t \in J$, which converge to $\tilde{h}$ and $\tilde{w}$ in the sense of 8.22 and 8.26 . Since Remark 6.3 guarantees that $\hat{\pi}$ is continuous for almost every $t$, in fact 8.5 holds for all $c \in I$, and the last task is to construct these approximations $\tilde{h}_{\epsilon}, \tilde{w}_{\epsilon}$. Seting $I=(a, b)$, we first let

$$
\check{w}_{\epsilon}(t, x)= \begin{cases}\int_{(a, x) \cap U_{\delta, \epsilon}(t)}\left(\tilde{h}^{\prime}(t, \xi)-h_{\epsilon}^{\prime}(t, \xi)+\mu_{\epsilon}(t)\right) \mathrm{d} y & \text { for } a<x<b, \\ 0 & \text { for } x \leqslant a \text { or } x \geqslant b,\end{cases}
$$

where $\mu_{\epsilon}(t)$ is to ensure that $\check{w}_{\epsilon}(t, b)=0$. We can also write $\check{w}_{\epsilon}$ as

$$
\begin{aligned}
\check{w}_{\epsilon}(t, x)= & \left(\tilde{h}(t, x)-h_{\epsilon}(t, x)\right)-\left(\tilde{h}(t, a)-h_{\epsilon}(t, a)\right) \\
& +\mathcal{L}^{1}\left((a, x) \cap U_{\delta, \epsilon}(t)\right) \mu_{\epsilon}(t)-\int_{(a, x)-U_{\delta, \epsilon}(t)} \mathrm{d}\left(\tilde{h}-h_{\epsilon}\right)(t) .
\end{aligned}
$$

From this we see that $\mu_{\epsilon}$ is given by

$$
\begin{aligned}
\mathcal{L}^{1}\left((a, b) \cap U_{\delta, \epsilon}(t)\right) \mu_{\epsilon}(t) & \\
& =\left(\tilde{h}(t, a)-h_{\epsilon}(t, a)\right)-\left(\tilde{h}(t, b)-h_{\epsilon}(t, b)\right)+\int_{(a, b)-U_{\delta, \epsilon}(t)} \mathrm{d}\left(\tilde{h}-h_{\epsilon}\right)(t) .
\end{aligned}
$$

Since, as established in (5.3), 4.4) and (4.5),

$$
\begin{aligned}
& h_{\epsilon} \stackrel{\epsilon \downarrow 0}{\longrightarrow} h \quad \text { locally uniformly in }[0, \infty) \times \mathbb{R}, \\
& \mathcal{L}^{1}\left(\mathbb{R}-U_{\delta, \epsilon}(t)\right) \stackrel{\epsilon \downarrow 0}{\longrightarrow} 0 \quad \text { uniformly in } t \in[0, \infty), \\
& \int_{\mathbb{R}-U_{\delta, \epsilon}(t)}\left|\mathrm{d} h_{\epsilon}(t)\right| \stackrel{\epsilon \downarrow 0}{\longrightarrow} 0 \quad \text { uniformly in } t \in[0, \infty),
\end{aligned}
$$


we obtain

$$
\mu_{\epsilon}(t) \stackrel{\epsilon \downarrow 0}{\longrightarrow} \frac{1}{b-a}[(\tilde{h}(t, a)-h(t, a))-(\tilde{h}(t, b)-h(t, b))]=0
$$

locally uniformly in $t \in[0, \infty)$. From $8.27-8.29$, we infer

$$
\check{w}_{\epsilon}(t, x) \stackrel{\epsilon \downarrow 0}{\longrightarrow}(\tilde{h}(t, x)-h(t, x))-(\tilde{h}(t, a)-h(t, a))=\tilde{w}(t, x)
$$

uniformly in $(t, x) \in J \times I$. We also observe for further reference that $\check{w}_{\epsilon}^{\prime}$ is uniformly bounded in $L^{2}(J \times I)$ :

$$
\int_{J \times I}\left(\check{w}_{\epsilon}^{\prime}\right)^{2} \leqslant \int_{J \times I}\left(\tilde{h}^{\prime}\right)^{2}+\int_{J} \int_{I \cap U_{\delta, \epsilon}(t)}\left(h_{\epsilon}^{\prime}\right)^{2}+\mathcal{L}^{1}(I) \int_{J}\left(\mu_{\epsilon}(t)\right)^{2} \leqslant C .
$$

In order to guarantee exact neutrality we need to introduce weights $A_{\epsilon}(t), B_{\epsilon}(t)$ so that

$$
\left\{\begin{aligned}
A_{\epsilon}(t) \int_{I}\left[\check{w}_{\epsilon}(t)\right]_{+} & -B_{\epsilon}(t) \int_{I}\left[\check{w}_{\epsilon}(t)\right]_{-} \\
A_{\epsilon}(t) & +B_{\epsilon}(t)=-\lambda_{\epsilon}(t),
\end{aligned}\right.
$$

Observing that, due to 8.25 ,

$$
\lambda_{\epsilon}(t)=v_{0}\left(y_{\epsilon}^{+}(t, c)-y_{\epsilon}^{-}(t, c)\right) \stackrel{\epsilon \downarrow 0}{\longrightarrow} v_{0} h(t, c)=-\int_{I} \tilde{w},
$$

in view of 8.30$)$ we have

$$
A_{\epsilon}(t), B_{\epsilon}(t) \stackrel{\epsilon \downarrow 0}{\longrightarrow} 1 \quad \text { uniformly in } J .
$$

Our Ansatz is

$$
\tilde{w}_{\epsilon}(t, x):=A_{\epsilon}(t)\left[\check{w}_{\epsilon}(t, x)\right]_{+}-B_{\epsilon}(t)\left[\check{w}_{\epsilon}(t, x)\right]_{-} .
$$

By 8.32 , each $\tilde{w}_{\epsilon}$ is neutral, i.e. satisfies 8.15 . In addition, by construction $\tilde{w}_{\epsilon}^{\prime} \in L^{2}((0, \infty) \times \mathbb{R})$ and

$$
\begin{array}{ll}
\tilde{w}_{\epsilon}^{\prime}(t)=0 & \text { a.e. outside } U_{\delta, \epsilon}(t), \\
\tilde{w}_{\epsilon}(t)=0 & \text { outside } I,
\end{array}
$$

for almost every $t \in J$. Finally, in view of 8.30 and 8.33 we obtain

$$
\tilde{w}_{\epsilon}(t, x) \stackrel{\epsilon \downarrow 0}{\longrightarrow} \tilde{w}(t, x) \quad \text { uniformly in }(t, x) \in J \times I,
$$

and therefore $\tilde{w}_{\epsilon}$ satisfy all the desired properties.

We are poised to define $\tilde{h}_{\epsilon}$ via

$$
\tilde{h}_{\epsilon}:=h_{\epsilon}+\tilde{w}_{\epsilon} .
$$

By (8.27) and 8.34

$$
\begin{aligned}
\tilde{h}_{\epsilon}(t, x)= & \tilde{h}(t, x)-\left(\tilde{h}(t, a)-h_{\epsilon}(t, a)\right) \\
& +\mathcal{L}^{1}\left((a, x) \cap U_{\delta, \epsilon}(t)\right) \mu_{\epsilon}(t)-\int_{(a, x)-U_{\delta, \epsilon}(t)} \mathrm{d}\left(\tilde{h}-h_{\epsilon}\right)(t) \\
& +\left(A_{\epsilon}(t)-1\right)\left[\check{w}_{\epsilon}(t, x)\right]_{+}-\left(B_{\epsilon}(t)-1\right)\left[\check{w}_{\epsilon}(t, x)\right]_{-} .
\end{aligned}
$$


From this representation and 8.31 we infer $\tilde{h}_{\epsilon}^{\prime} \in L^{2}\left(U_{\delta, \epsilon}\right)$ and

$$
\tilde{h}_{\epsilon}^{\prime}=\tilde{h}^{\prime}+\mu_{\epsilon}+\left[\left(A_{\epsilon}-1\right) \chi_{\left\{\check{w}_{\epsilon}>0\right\}}-\left(B_{\epsilon}-1\right) \chi_{\left\{\check{w}_{\epsilon}<0\right\}}\right] \check{w}_{\epsilon}^{\prime} \quad \text { a.e. in } U_{\delta, \epsilon} .
$$

Hence, by 8.29, , 8.31, and 8.33 we obtain as desired

$$
\left\{\begin{array}{ll}
\tilde{h}_{\epsilon}^{\prime} & \text { in } U_{\delta, \epsilon} \\
0 & \text { elsewhere }
\end{array}\right\} \stackrel{\epsilon \downarrow 0}{\longrightarrow} \tilde{h}^{\prime} \quad \text { in } L^{2}(J \times I),
$$

and the proof is complete.

\section{Recovering the equation}

Proposition 8.1 with $v_{0}=0$ is already sufficient to make the identification $v=h^{\prime \prime \prime}$ on the positivity set. Indeed, we have the following:

PROPOSITION 9.1 For almost every $t>0$ and for every connected component $(a, b)$ of $\{h(t)>0\}$, we have

$$
v(t)=h^{\prime \prime \prime}(t) \text { in } L_{\mathrm{loc}}^{2}((a, b)) .
$$

In addition

$$
\int_{\mathbb{R}} h(t) h^{\prime \prime \prime}(t) \tilde{v}=\int_{\mathbb{R}}\left\{\frac{3}{2}\left(h^{\prime}(t)\right)^{2} \tilde{v}^{\prime}+h(t) h^{\prime}(t) \tilde{v}^{\prime \prime}\right\}
$$

for all $\tilde{v} \in H_{c}^{2}((a, b))$.

REMARK 9.2 The identification in (9.1), together with (6.9) and (6.10), establishes part (ii) in Definition 1. and already implies that $h$ solves the thin-film equation in the weak sense of 2.3):

$$
\int_{0}^{\infty} \int h \zeta_{t}+\int_{0}^{\infty} \int_{\{h(t)>0\}} h h^{\prime \prime \prime} \zeta^{\prime}=0 \quad \text { for all } \zeta \in C_{c}^{\infty}\left(\mathbb{R}^{+} \times \mathbb{R}\right) .
$$

Proof. Identity 9.2) follows immediately from 9.1) after two integrations by parts. To prove 9.1, we fix a time $t>0$ such that, in view of (5.5), 6.9) and 6.11,

$$
\int_{\mathbb{R}}\left(h^{\prime}(t)\right)^{2}<\infty, \quad-\hat{\pi}^{\prime}(t)=v(t) \quad \text { in } L_{\mathrm{loc}}^{2}((a, b)) .
$$

For any $\tilde{v} \in C_{c}^{\infty}((a, b))$ and any $\tau>0$, we let $\tilde{w}=\tau \tilde{v}^{\prime}$ as test function in 8.1 :

$$
\frac{1}{2 \tau} \int_{a}^{b}\left[\left(h^{\prime}(t)+\tau \tilde{v}^{\prime \prime}\right)^{2}-\left(h^{\prime}(t)\right)^{2}\right] \geqslant \int_{a}^{b} \hat{\pi} \tilde{v}^{\prime} \stackrel{\text { 9.4. }}{=} \int_{a}^{b} v \tilde{v} .
$$

Sending $\tau$ to zero yields

$$
\int_{a}^{b} h^{\prime}(t) \tilde{v}^{\prime \prime} \geqslant \int_{a}^{b} v \tilde{v}
$$

Exchanging $\tilde{v}$ with $-\tilde{v}$ we see that equality holds, and the proof is complete. 


\section{Post-processing I: Regularity and zero contact-angle}

In this section we recover the free boundary condition:

$$
\begin{gathered}
h(t) \text { is continuously differentiable on } \overline{\{h(t)>0\}} \text { with } \\
h^{\prime}(t)=0 \quad \text { at } \partial\{h(t)>0\} \quad \text { for a.e. } t \in(0, \infty) .
\end{gathered}
$$

Proposition 10.1 For almost every $t>0$ and for every connected component $(a, b)$ of $\{h(t)>0\}$, we have

$$
\sup _{x \neq y \in(a, b)} \frac{\left|h^{\prime}(t, x)-h^{\prime}(t, y)\right|}{|x-y|^{2 / 3}} \leqslant C \int_{a}^{b}\left|h^{\prime \prime}(t)\right|^{3} \leqslant C \int_{a}^{b} h(t)\left(h^{\prime \prime \prime}(t)\right)^{2}<\infty
$$

with $C$ a universal constant, and $h(t, \cdot)$ is continuously differentiable on $[a, b]$ with

$$
h^{\prime}(t, \cdot)=0 \quad \text { at }\{a, b\} .
$$

REMARK 10.2 The combination of 10.1 and 10.2 immediately gives the regularity property $h_{x x} \in L^{3}((0, \infty) \times \mathbb{R})$ stated in Definition 1 (iii).

By reasons of symmetry, we only consider the right end-point $b$. The proof is split into three lemmas. First we show that test functions $\tilde{v}$ which do not vanish at $b$ are in fact admissible in 9.2 , provided they correspond to a "stretching" of the film:

LEMMA 10.3 For almost every $t>0$ and for every connected component $(a, b)$ of $\{h(t)>0\}$, we have

$$
\int_{c_{0}}^{b}\left\{\frac{3}{2}\left(h^{\prime}(t)\right)^{2} \tilde{v}^{\prime}+h(t) h^{\prime}(t) \tilde{v}^{\prime \prime}\right\} \leqslant \int_{c_{0}}^{b} h(t) h^{\prime \prime \prime}(t) \tilde{v}
$$

for any $c_{0} \in(a, b)$ such that

$$
h^{\prime}\left(t, c_{0}\right)=0
$$

and every $\tilde{v} \in H^{2}(\mathbb{R})$ such that

$$
\left.\begin{array}{rl}
\operatorname{supp}(\tilde{v}) & \subset\left(c_{0}, \infty\right) \\
\operatorname{supp}\left(\tilde{v}^{\prime}\right) & \subset\left(c_{0}, b\right) \\
\tilde{v}(b) & \geqslant 0
\end{array}\right\}
$$

This, by a suitable choice of $\tilde{v}$, allows us to infer the zero contact-angle condition in the following weak sense:

LEMMA 10.4 For almost every $t>0$ and for every connected component $(a, b)$ of $\{h(t)>0\}$, there exists a sequence $b_{n} \uparrow b$ such that

$$
\lim _{n \rightarrow \infty} h^{\prime}\left(t, b_{n}\right)=0
$$

Such weak notion is however sufficient to infer the aforementioned regularity properties for the solution. This is a consequence of the following extension of Bernis' estimates [4]: 
Lemma 10.5 Let $-\infty \leqslant a<b \leqslant \infty$. There exists a universal constant $C_{0}$ such that

$$
\begin{array}{r}
\int_{a}^{b}\left|h^{\prime \prime}\right|^{3} \mathrm{~d} x \leqslant C_{0} \int_{a}^{b} h\left(h^{\prime \prime \prime}\right)^{2} \mathrm{~d} x, \\
\int_{a}^{b} h^{-3}\left(h^{\prime}\right)^{6} \mathrm{~d} x \leqslant C_{0} \int_{a}^{b} h\left(h^{\prime \prime \prime}\right)^{2} \mathrm{~d} x
\end{array}
$$

for any function $h$ satisfying:

(A) $h \in H_{\mathrm{loc}}^{3}((a, b)) \cap C([a, b]), h>0$ in $(a, b), h^{\prime} \in L^{2}((a, b))$;

(B) there exist sequences $a_{m} \downarrow a, b_{m} \uparrow b$ such that $h^{\prime}\left(a_{n}\right) \rightarrow 0, h^{\prime}\left(b_{n}\right) \rightarrow 0$;

(C) $\int_{a}^{b} h\left(h^{\prime \prime \prime}\right)^{2} \mathrm{~d} x<\infty$.

This result is stated and proved in [12] for more general non-linearities (the additional assumption $h \in C^{3}((a, b))$ in that statement is harmless). Bernis's estimates imply 10.1 at once (the first inequality is in fact a standard embedding), and combining (10.1) with Lemma 10.4 one obtains the strong form of zero contact-angle condition stated in Proposition 10.1 .

The rest of the section is concerned with the proof of Lemmas 10.3 and 10.4 , during which we shall consider a fixed $t>0$ such that (8.1) holds and, in view of (5.5), 6.9), 6.11) and (9.1),

$$
\int_{a}^{b}\left(h^{\prime}(t)\right)^{2}<\infty, \quad \int_{a}^{b} h(t)\left(h^{\prime \prime \prime}(t)\right)^{2}<\infty, \quad-\hat{\pi}^{\prime}(t)=h^{\prime \prime \prime}(t) \text { in } L_{\mathrm{loc}}^{2}((a, b)),
$$

omitting the $t$ variable for notational convenience.

Proof of Lemma 10.3 According to (10.4), the function

$$
\bar{v}(x):=\tilde{v}(b)-\tilde{v}(x)
$$

is such that

$$
\begin{aligned}
\operatorname{supp}(\bar{v}) & \subset(-\infty, b), \\
\operatorname{supp}\left(\bar{v}^{\prime}\right) & \subset\left(c_{0}, b\right), \\
\bar{v}\left(c_{0}\right) & =\tilde{v}(b) \geqslant 0 .
\end{aligned}
$$

For $\tau>0$ we define

$$
\tilde{w}_{\tau}(x):=\left\{\begin{array}{ll}
0, & x \leqslant c_{0}, \\
\tau(h(x) \bar{v}(x))^{\prime}, & x>c_{0},
\end{array} \quad \tilde{h}_{\tau}:=h+\tilde{w}_{\tau} .\right.
$$

We wish to choose $\tilde{h}_{\tau}, \tilde{w}_{\tau}$ as test functions in 8.1 . We have $\tilde{w}_{\tau} \in H^{1}\left(\left(c_{0}, \infty\right)\right)$ since, by 10.8 , resp. 9.1$], \operatorname{supp}\left(\tilde{w}_{\tau}\right) \subset\left[c_{0}, b\right)$ and $h \in H_{\mathrm{loc}}^{3}((a, b))$. In addition, in view of 10.3 and 10.9 ,

$$
\lim _{x \rightarrow c_{0}^{+}} \tilde{w}_{\tau}(x)=\tau h^{\prime}\left(c_{0}\right) \bar{v}\left(c_{0}\right)+\tau h\left(c_{0}\right) \bar{v}^{\prime}\left(c_{0}\right)=0 .
$$

Therefore

$$
\tilde{w}_{\tau} \in H^{1}(\mathbb{R}), \quad \operatorname{supp}\left(\tilde{w}_{\tau}\right) \subset\left[c_{0}, b\right) \subset(a, b) .
$$


The "neutrality" condition 8.4 is guaranteed by an appropriate choice of the constant $v_{0}=v_{0 \tau}$ :

$$
\int_{\mathbb{R}} \tilde{w}_{\tau}=-\tau h\left(c_{0}\right) \bar{v}\left(c_{0}\right)=-v_{0 \tau} h\left(c_{0}\right),
$$

where

$$
v_{0 \tau}:=\tau \bar{v}\left(c_{0}\right)^{10.10} \tau \tilde{v}(b) .
$$

In view of 10.11 and 10.12, $\tilde{h}_{\tau}$ and $\tilde{w}_{\tau}$ are admissible tests in 8.1 with $v_{0}=v_{0 \tau}$ and $I$ such that $\operatorname{supp}\left(\tilde{w}_{\tau}\right) \subset I \subset(a, b), c_{0} \in I$. Dividing the inequality by $\tau$ and integrating by parts yields

$$
\begin{aligned}
\frac{1}{2 \tau} \int_{c_{0}}^{b}\left\{\left(\tilde{h}_{\tau}^{\prime}\right)^{2}-\left(h^{\prime}\right)^{2}\right\} & \geqslant-\frac{v_{0 \tau}}{\tau} \int_{c_{0}}^{b} h v+\int_{c_{0}}^{b} \hat{\pi}(h \bar{v})^{\prime}+\frac{v_{0 \tau}}{\tau} h\left(c_{0}\right) \hat{\pi}\left(c_{0}\right) \\
& \stackrel{10.13}{=}-\int_{c_{0}}^{b} h v \tilde{v}(b)+[\hat{\pi} h \bar{v}]_{c_{0}}^{b}-\int_{c_{0}}^{b} h \hat{\pi}^{\prime} \bar{v}+\bar{v}\left(c_{0}\right) h\left(c_{0}\right) \hat{\pi}\left(c_{0}\right) \\
& 10.7 \\
= & \int_{c_{0}}^{b} h h^{\prime \prime \prime}(\bar{v}-\tilde{v}(b))=-\int_{c_{0}}^{b} h h^{\prime \prime \prime} \tilde{v} .
\end{aligned}
$$

Passing to the limit with respect to $\tau$ gives

$$
\int_{c_{0}}^{b} h^{\prime}(h \bar{v})^{\prime \prime} \geqslant-\int_{c_{0}}^{b} h h^{\prime \prime \prime} \tilde{v} .
$$

An integration by parts on the left hand side yields

$$
\begin{aligned}
\int_{c_{0}}^{b} h^{\prime}(h \bar{v})^{\prime \prime} & =\int_{c_{0}}^{b}\left\{\left[\frac{1}{2}\left(h^{\prime}\right)^{2}\right]^{\prime} \bar{v}+2\left(h^{\prime}\right)^{2} \bar{v}^{\prime}+h h^{\prime} \bar{v}^{\prime \prime}\right\} \\
& =-\int_{c_{0}}^{b}\left\{\frac{3}{2}\left(h^{\prime}\right)^{2} \tilde{v}^{\prime}+h h^{\prime} \tilde{v}^{\prime \prime}\right\}
\end{aligned}
$$

and the proof is complete.

Proof of Lemma 10.4 For fixed $x \in\left(c_{0}, b\right)$, we choose in Lemma 10.3 a test function $\tilde{v}$ such that

$$
\operatorname{supp}(\tilde{v}) \subset\left(c_{0}, \infty\right), \quad \operatorname{supp}\left(\tilde{v}^{\prime}\right) \subset\left(c_{0}, x\right), \quad \tilde{v}(\xi) \equiv 1 \text { for } \xi \geqslant x
$$

Integrating by parts (all integrations are admissible since $h \in H_{\text {loc }}^{3}((a, b))$ ), with this choice we obtain

$$
\begin{aligned}
\int_{c_{0}}^{b} h h^{\prime \prime \prime} \tilde{v} & \geqslant \int_{c_{0}}^{x}\left\{\frac{3}{2}\left(h^{\prime}\right)^{2} \tilde{v}^{\prime}+h h^{\prime} \tilde{v}^{\prime \prime}\right\} \\
& =\frac{3}{2}\left(h^{\prime}(x)\right)^{2}-\int_{c_{0}}^{x}\left\{3 h^{\prime} h^{\prime \prime} \tilde{v}+\left(h^{\prime}\right)^{2} \tilde{v}^{\prime}+h h^{\prime \prime} \tilde{v}^{\prime}\right\} \\
& =\frac{1}{2}\left(h^{\prime}(x)\right)^{2}-h(x) h^{\prime \prime}(x)+\int_{c_{0}}^{x} h h^{\prime \prime \prime} \tilde{v}
\end{aligned}
$$


and therefore, by 10.14 and 10.7),

$$
\frac{1}{2}\left(h^{\prime}(x)\right)^{2} \leqslant h(x) h^{\prime \prime}(x)+\int_{x}^{b} h h^{\prime \prime \prime}=h(x) h^{\prime \prime}(x)+o_{|b-x|}(1) \quad \forall x \in\left(c_{0}, b\right) .
$$

We now reason by contradiction and assume that there exist positive $\tilde{\delta}$ and $C$ with

$$
\left(h^{\prime}\right)^{2} \geqslant C^{2}>0 \quad \text { on }[b-\tilde{\delta}, b) .
$$

Then (10.15) yields the differential inequality

$$
h^{\prime \prime}(x) \geqslant \frac{C^{2}}{2 h(x)}-\frac{o_{|b-x|}(1)}{h(x)} \geqslant \frac{C^{2}}{4 h(x)} \text { for all } x \in[b-\delta, b)
$$

for $\delta \leqslant \tilde{\delta}$ sufficiently small. Because of $h(b)=0,10.16$ in particular implies $h^{\prime}<0$ on $[b-\delta, b)$. Multiplying 10.17 with $h^{\prime}$, we obtain

$$
\left[\left(h^{\prime}\right)^{2}\right]^{\prime} \leqslant \frac{C^{2}}{2}(\ln h)^{\prime} \quad \text { on }[b-\delta, b) .
$$

We integrate this inequality over $(b-\delta, x)$ for some $x \in(b-\delta, b)$ and obtain

$$
-\left(h^{\prime}(b-\delta)\right)^{2} \leqslant \frac{C^{2}}{2}(\ln h(x)-\ln h(b-\delta)) .
$$

From this inequality, for $x \rightarrow b$ we obtain a contradiction to $h(b)=0$. Thus 10.16 is false and the proof is complete.

\section{Post-processing II: The "Neumann-type" formulation}

The aim of this last section is to complete the proof of Theorem 2 recovering the "Neumanntype" formulation (2.2) for the thin-film evolution which encompasses both the PDE and the freeboundary condition. Namely, we have the following:

Proposition 11.1 For almost every $t>0$ and every connected component $(a, b)$ of $\{h(t)>0\}$, we have

$$
\int_{a}^{b}\left\{\frac{3}{2}\left(h^{\prime}(t)\right)^{2} \tilde{v}^{\prime}+h(t) h^{\prime}(t) \tilde{v}^{\prime \prime}\right\}=\int_{a}^{b} h(t) h^{\prime \prime \prime}(t) \tilde{v}
$$

for all $v \in H_{c}^{2}(\mathbb{R})$.

REMARK 11.2 Combining (11.1) with 9.3) we immediately obtain Definition[1(iv), and the proof of Theorem 2 is complete.

Proof. We neglect time dependence, and work for a fixed $t$ such that for every connected component $(a, b)$ of $\{h>0\}$ (cf. Proposition 10.1 .

$$
h^{\prime}(a)=h^{\prime}(b)=0, \quad \int_{\mathbb{R}}\left(h^{\prime}\right)^{2}<\infty
$$


and

$$
\sup _{x \neq y \in(a, b)} \frac{\left|h^{\prime}(x)-h^{\prime}(y)\right|}{|x-y|^{2 / 3}} \leqslant C \int_{a}^{b}\left|h^{\prime \prime}\right|^{3} \leqslant C \int_{a}^{b} h\left(h^{\prime \prime \prime}\right)^{2}<\infty .
$$

Given $\tilde{v} \in H_{c}^{2}(\mathbb{R})$, we let

$$
\tilde{v}_{\delta}=\tilde{v} \varphi_{\delta}
$$

where $\varphi_{\delta}$ is such that

$$
\begin{aligned}
& \operatorname{supp}\left(\varphi_{\delta}\right)=[a+\delta, b-\delta], \\
& \varphi_{\delta} \equiv 1 \quad \text { in }(a+2 \delta, b-2 \delta), \\
& \left|\varphi_{\delta}^{(j)}\right| \leqslant C_{0} \delta^{-j}, \quad j=1,2 .
\end{aligned}
$$

The functions $\tilde{v}_{\delta}$ are admissible tests in 9.2):

$$
\int_{\mathbb{R}}\left\{\frac{3}{2}\left(h^{\prime}\right)^{2} \tilde{v}_{\delta}^{\prime}+h h^{\prime} \tilde{v}_{\delta}^{\prime \prime}\right\}=\int_{\mathbb{R}} h h^{\prime \prime \prime} \tilde{v}_{\delta} .
$$

We wish to recover (11.1) by passing to the limit as $\delta \downarrow 0$ in 11.4 . The right hand side is trivially convergent. For the first term on the left hand side we write

$$
\int_{\mathbb{R}}\left(h^{\prime}\right)^{2} \tilde{v}_{\delta}^{\prime}=\int_{\mathbb{R}}\left(h^{\prime}\right)^{2} \tilde{v}^{\prime}+\int_{\mathbb{R}}\left(h^{\prime}\right)^{2} \tilde{v}^{\prime}\left(\varphi_{\delta}-1\right)+\int_{\mathbb{R}}\left(h^{\prime}\right)^{2} \tilde{v} \varphi_{\delta}^{\prime} .
$$

It follows from (11.2) and (11.3) that there exists $C<\infty$ such that

$$
\begin{aligned}
& \sup _{x \in \operatorname{supp}\left(\varphi_{\delta}\right)}\left|h^{\prime}(x)\right| \leqslant \sup _{x \in(a, a+2 \delta) \cup(b-2 \delta, b)}\left|h^{\prime}(x)\right| \leqslant C \delta^{2 / 3}, \\
& \sup _{x \in \operatorname{supp}\left(\varphi_{\delta}\right)}|h(x)| \leqslant \sup _{x \in(a, a+2 \delta) \cup(b-2 \delta, b)}|h(x)| \leqslant C \delta^{5 / 3} .
\end{aligned}
$$

Thus we obtain at once

$$
\begin{array}{r}
\left|\int_{\mathbb{R}}\left(h^{\prime}\right)^{2} \tilde{v}^{\prime}\left(\varphi_{\delta}-1\right)\right| \leqslant \delta^{4 / 3} \int_{a}^{b}\left|\tilde{v}^{\prime}\right| \stackrel{\delta \downarrow 0}{\longrightarrow} 0, \\
\int_{\mathbb{R}}\left(h^{\prime}\right)^{2}\left|\tilde{v} \varphi_{\delta}^{\prime}\right| \leqslant \delta^{1 / 3} \int_{a}^{b}|\tilde{v}| \stackrel{\delta \downarrow 0}{\longrightarrow} 0 .
\end{array}
$$

For the second term on the left hand side we write

$$
\int_{\mathbb{R}} h h^{\prime} \tilde{v}_{\delta}^{\prime \prime}=\int_{\mathbb{R}} h h^{\prime} \tilde{v}^{\prime \prime}+\int_{\mathbb{R}} h h^{\prime} \tilde{v}^{\prime \prime}\left(\varphi_{\delta}-1\right)+2 \int_{\mathbb{R}} h h^{\prime} \tilde{v}^{\prime} \varphi_{\delta}^{\prime}+\int_{\mathbb{R}} h h^{\prime} \tilde{v} \varphi_{\delta}^{\prime \prime} .
$$

By 11.5 ) and 11.6,

$$
\begin{array}{r}
\left|\int_{\mathbb{R}} h h^{\prime} \tilde{v}^{\prime \prime}\left(\varphi_{\delta}-1\right)\right| \leqslant C \delta^{7 / 3} \int_{a}^{b}\left|\tilde{v}^{\prime \prime}\right| \stackrel{\delta \downarrow 0}{\longrightarrow} 0, \\
\int_{\mathbb{R}}\left|h h^{\prime} \tilde{v}^{\prime} \varphi_{\delta}^{\prime}\right| \leqslant C \delta^{4 / 3} \int_{a}^{b}\left|\tilde{v}^{\prime}\right| \stackrel{\delta \downarrow 0}{\longrightarrow} 0, \\
\int_{\mathbb{R}}\left|h h^{\prime} \tilde{v} \varphi_{\delta}^{\prime \prime}\right| \leqslant C \delta^{1 / 3} \int_{a}^{b}|\tilde{v}| \stackrel{\delta \downarrow 0}{\longrightarrow} 0,
\end{array}
$$

and the proof is complete. 


\section{Acknowledgements}

We acknowledge support of SFB 611 "Singularities and scaling in mathematical models" of the University of Bonn, and of E.C. through the TMR-Programme "Nonlinear parabolic partial differential equations: Methods and applications" (FMRX-CT98-0201) and the RTNProgramme "Nonlinear partial differential equations describing front propagation and other singular phenomena" (HPRN-CT-2002-00274). L.G. acknowledges the kind hospitality of the Institut für Angewandte Mathematik in Bonn. It is a pleasure to thank one of the referees for having suggested us a simplification in Section 4.

\section{REFERENCES}

1. Almgren, R. Singularity formation in Hele-Shaw bubbles. Phys. Fluids 8 (1996), 344-352. MR 96j:76055

2. Almgren, R., Bertozzi, A., \& Brenner, M. P. Stable and unstable singularities in the unforced Hele-Shaw cell. Phys. Fluids 8 (1996), 1356-1370. MR 97e:76038

3. Beretta, E., Bertsch, M., \& Dal Passo, R. Nonnegative solutions of a fourth-order nonlinear degenerate parabolic equation. Arch. Rat. Mech. Anal. 129 (1995), 175-200. Zbl 0827.35065 MR 96b:35116

4. BERNIS, F. Integral inequalities with applications to nonlinear degenerate parabolic equations. Nonlinear Boundary Value Problems, T. S. Angell, L. P. Cook, R. E. Kleinman and W. E. Olmstead (eds.), SIAM, Philadelphia (1996), 57-65. Zbl 0889.35054

5. BERNIS, F. Finite speed of propagation and continuity of the interface for thin viscous flows. $A d v$. Differential Equations 1 (1996), 337-368. Zbl 0846.35058 MR 97e:35095

6. Bertozzi, A. L. \& Pugh, M. The lubrication approximation for thin viscous films: regularity and long time behaviour of weak solutions. Comm. Pure Appl. Math. 49 (1994), 85-123. Zbl 0863.76017 MR 97b:35114

7. Constantin, P. \& PUgh, M. Global solutions for small data to the Hele-Shaw problem. Nonlinearity 6 (1993), 393-415. Zbl 0808.35104 MR 94j:35142

8. Duchon, J. \& Robert, R. Évolution d'une interface par capillarité et diffusion de volume. I. Existence locale en temps. Ann. Inst. H. Poincaré Anal. Non Linéaire 1 (1984), 361-378. Zbbl 0572.35051 MR 86i:35133

9. Escher, J. \& Simonett, G. Classical solutions for Hele-Shaw models with surface tension. Adv. Differential Equations 2 (1997), 619-642. MR 98b:35204

10. De Gennes, P. G. Wetting: Statics and dynamics. Rev. Mod. Phys. 57 (1985), 827-863.

11. Giacomelli, L. \& OTto, F. Variational formulation for the lubrication approximation of the Hele-Shaw flow. Calc. Var. Partial Differential Equations 13 (2001), 377-403. Zbl pre01703144 MR 2003a:76046

12. Giacomelli, L. \& OTto, F. Droplet spreading: intermediate scaling law by PDE methods. Comm. Pure Appl. Math. 55 (2002), 217-254. Zbl pre01860563 MR 2002g:76047

13. GRÜN, G. Droplet spreading under weak slippage: the optimal asymptotic propagation rate in the multidimensional case. Interfaces Free Bound. 4 (2002), 309-323. Zbl pre01791242

14. Oron, A., Davis, S. H. \& Bankoff, G. Long-scale evolution of thin liquid films. Rev. Mod. Phys. 69 (1997), 931-980.

15. Отто, F. Lubrication approximation with prescribed non-zero contact angle. Comm. Partial Differential Equations 23 (1998), 2077-2164. Zbl 0923.35211 MR 99m:35276

16. Prokert, G. Existence results for Hele-Shaw flow driven by surface tension. Euro. J. Appl. Math. 9 (1998), 195-221. Zbl 0919.35005 MR 2000d:76052 